\section{UCDNN}

LIBRARY
University of Connecticut OpenCommons@UConn

8-6-2014

\title{
Scattering of Particles and Radiation in Astrophysical Environments
}

Nicholas R. Lewkow

lewkow@phys.uconn.edu

Follow this and additional works at: https://opencommons.uconn.edu/dissertations

\section{Recommended Citation}

Lewkow, Nicholas R., "Scattering of Particles and Radiation in Astrophysical Environments" (2014). Doctoral Dissertations. 511.

https://opencommons.uconn.edu/dissertations/511 


\section{Scattering of Particles and Radiation in Astrophysical Environments}

Nicholas R. Lewkow, Ph.D.

University of Connecticut, 2014

The scattering of atoms, molecules, and radiation are the most fundamental mechanisms of energy-momentum transfer in nature. Fluxes of particles and radiation may deposit energy into a system as well as be emitted, giving deep insight into fundamental processes occurring within the system. Astrophysics, in particular, may benefit from collisional modeling and transport as remote viewing of distant objects is the only option for the vast majority of astrophysical objects. Practically all interaction processes between atoms, molecules, and radiation require a quantum mechanical description. Using well known quantum analysis as well as newly developed methods, scattering parameters have been obtained over important astrophysical energy ranges not previously investigated. The newly calculated parameters have been used to model transport, collisional heating, and thermalization in a variety of astrophysical systems including the atmosphere of Mars, cometary atmospheres, and the interstellar gas and plasmas. Results of the collisional simulations allow for better understanding of current observational data as well as predictions for next generation in situ measurements. 


\title{
Scattering of Particles and Radiation in Astrophysical Environments
}

\author{
Nicholas R. Lewkow \\ B.S., Colorado State University, 2009 \\ M.S., University of Connecticut, 2011
}

\author{
A Dissertation \\ Submitted in Partial Fullfilment of the \\ Requirements for the Degree of \\ Doctor of Philosophy \\ at the \\ University of Connecticut
}

2014 
Copyright by

Nicholas R. Lewkow 


\title{
APPROVAL PAGE
}

Doctor of Philosophy Dissertation

\section{Scattering of Particles and Radiation in Astrophysical}

\section{Environments}

\section{Presented by}

Nicholas R. Lewkow, B.S., M.S.

Major Advisor

Vasili Kharchenko

Associate Advisor

$$
\text { Robin Côté }
$$

Associate Advisor

\author{
Thomas Blum
}

University of Connecticut

2014 
I dedicate this work to Merry Lee Lewkow for always believing in me and pushing me to realize my potential. 


\section{ACKNOWLEDGEMENTS}

It is a long hard road to complete a Ph.D. and it could not be done alone. I would like to thank my advisor Dr. Vasili Kharchenko for all his help and guidance through the past years. From discussing planetary atmospheres, to quantum phenomena, to teaching me how to play tennis, Vasili has been not only a great mentor but a great friend. Additionally, I would like to thank my co-advisors, Dr. Thomas Blum and Dr. Robin Cote. Their help and guidance throughout my dissertation writing and editing has been invaluable. My family has also been a great source of support throughout graduate school. Specifically, I would like to thank my father Kenneth Lewkow for installing an excellent work ethic in me as a young child and always helping me when school was difficult and enthusiasm was low. My brother Timothy Lewkow has also been a great source of support as well as a great friend who may easily discuss complex math concepts over late night conversations. Additionally, Dr. Elizabeth Sather and Genny Ray have been extremely supportive through the years, helping me keep tabs on my family in Colorado while studying on the east coast. I would also like to thank

all my fellow graduate students and postdoctoral students who have been great friends, study buddies, and colleagues. 


\section{TABLE OF CONTENTS}

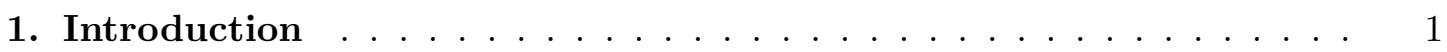

2. Particle Scattering in Astrophysical Environments . . . . . . . . 4

2.1 Collisional Cross Sections . . . . . . . . . . . . . . . 5

2.1 .1 Partial Wave Scattering . . . . . . . . . . . . . . . . 5

2.1 .2 Interaction Potentials $\ldots \ldots \ldots \ldots \ldots \ldots$

2.1.3 Partial Wave Cross Sections . . . . . . . . . . . . . . . . . . 20

2.1.4 Empirical Scaling Cross Sections _. . . . . . . . . . . . . . . . . 29

2.1.5 Charge Exchange Cross Sections . . . . . . . . . . . . . . 36

2.2 Monte Carlo Transport _ . . . . . . . . . . . . . . . . . . . . . . 44

$2.2 .1 \quad$ Ion Transport . . . . . . . . . . . . . . . . . . . . . 51

2.2.2 Frame Transformations _. . . . . . . . . . . . . . 57

2.3 Precipitation of Energetic Neutral Atoms in the Atmosphere of Mars . . . . 60

2.4 Transport of Energetic Neutral Atoms in the Local Interstellar Medium . 97

3. X-ray Scattering by Nanoparticles . . . . . . . . . . . . . . . . . . . 114

3.1 Radiation Scattering Cross Sections _. . . . . . . . . . . . 115

3.2 Heliosphere Dust Scattering . . . . . . . . . . . . . . . . . 125

3.3 X-ray Scattering from Cometary Atmospheres . . . . . . . . . . . . . . . . 129

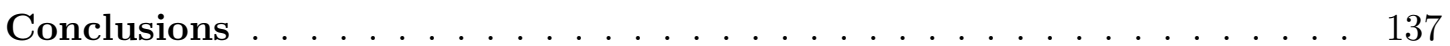

$\begin{array}{lr}\text { Bibliography } & 139\end{array}$ 


\section{LIST OF FIGURES}

2.1 Quantum mechanical partial wave scattering . . . . . . . . . 6

2.2 Partial wave convergence . . . . . . . . . . . . . . 15

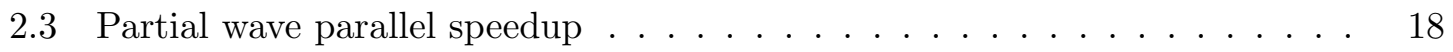

$2.4 \quad$ Ab initio interaction potentials . . . . . . . . . . . . . . . . . 19

$2.5 \mathrm{He}+\mathrm{He}$ differential cross sections . . . . . . . . . . . . . . . . 21

$2.6 \mathrm{He}+\mathrm{O}$ differential cross sections . . . . . . . . . . . . . . . 22

$2.7 \mathrm{He}+\mathrm{H}$ differential cross sections . . . . . . . . . . . . . . . . 23

$2.8 \mathrm{H}+\mathrm{H}$ differential cross sections . . . . . . . . . . . . . . . 24

2.9 Partial wave total cross sections . . . . . . . . . . . . . 25

2.10 Scattering angle probability densities . . . . . . . . . . . . 27

2.11 Cumulative scattering angle probability density . . . . . . . . . . 28

2.12 Average energy loss per collision f . . . . . . . . . . . . . . 28

2.13 Differential cross sections in reduced coordinates . . . . . . . . . . . . . 31

2.14 Quantum scaling cross sections with experimental atom-molecule data . . . 32

2.15 Quantum scaling differential cross sections . . . . . . . . . . . . 34

$2.16 \mathrm{H}^{+} \mathrm{CX}$ cross sections $\ldots \ldots \ldots \ldots \ldots \ldots$

$2.17 \mathrm{He}^{2+}$ and $\mathrm{He}^{+} \mathrm{CX}$ cross sections $\ldots \ldots \ldots \ldots \ldots$

2.18 Monte Carlo transport flow diagram . . . . . . . . . . . . . . . 45

2.19 Scattering angle probability distribution functions for $\mathrm{He}+\mathrm{H} \quad \ldots \ldots$. . . 47

2.20 Diagram for neutral and ion transport . . . . . . . . . . . . 52 
2.21 Ion drag diagram $\ldots \ldots \ldots \ldots \ldots \ldots \ldots \ldots$

2.22 Distance dependent energy loss due to ion drag . . . . . . . . . . . . 56

2.23 Collision reference frame diagram . . . . . . . . . . . . . . . . 58

2.24 Minimum solar activity Mars atmosphere model . . . . . . . . . . . . . 65

2.25 Mean solar activity Mars atmosphere model . . . . . . . . . . . . . . . 66

2.26 Maximum solar activity Mars atmosphere model . . . . . . . . . . . . . . 67

2.27 Quantum scaling total cross sections . . . . . . . . . . . . . . . 69

2.28 Quantum scaling average scattering angles . . . . . . . . . . . . . 71

2.29 Energetic neutral atom production . . . . . . . . . . . . . 74

2.30 Time dependent energy distributions . . . . . . . . . . . . . 78

2.31 Average, altitude dependent energy distributions . . . . . . . . . . . . 80

2.32 Thermalization altitudes . . . . . . . . . . . . . . . . . . 82

2.33 Thermalization times . . . . . . . . . . . . . . . . . 84

2.34 Secondary hot atom $/$ molecule production . . . . . . . . . . . . 86

2.35 Secondary hot atom $/$ molecule energy distributions $\ldots \ldots \ldots$. . . . . 88

2.36 Reflected energetic neutral atom energy distributions . . . . . . . . . . . 96

2.37 IBEX high energy all sky maps . . . . . . . . . . . . . . . . . . . . 99

2.38 IBEX low energy all sky maps f . . . . . . . . . . . . . . . 100

2.39 Neutral atomic density in the local interstellar medium . . . . . . . . . . 101

2.40 Mean free paths in local interstellar cloud . . . . . . . . . . . . . . . . 104

2.41 Mean free paths in local bubble . . . . . . . . . . . . . . 105

2.42 Single star ENA transport . . . . . . . . . . . . . . . . 106 
2.43 3D map of the 60 stars closest to the Sun . . . . . . . . . . . . 107

2.44 Steady state helium fluxes originating from nearby stars . . . . . . . . . . 110

2.45 Steady state helium fluxes originating from the LIC-LB boundary . . . . . 111

3.1 Real components of complex indices of refraction . . . . . . . . . . . 118

3.2 Imaginary components of complex indices of refraction . . . . . . . . . 119

3.3 Differential cross sections for X-ray scattering from carbon grains . . . . . . 120

3.4 Differential cross sections for X-ray scattering from silicon grains . . . . . . 121

3.5 Differential cross sections for X-ray scattering from ice grains . . . . . . . . 122

3.6 Total cross sections for X-ray scattering from $1 \mathrm{~nm}$ grains . . . . . . . . 123

3.7 Total cross sections for X-ray scattering from $5 \mathrm{~nm}$ grains . . . . . . . . 124

3.8 Total cross sections for X-ray scattering from $10 \mathrm{~nm}$ grains . . . . . . . . . 124

3.9 Sun-nanoparticle-detector scattering diagram f . . . . . . . . . . . . 127

3.10 Nanoparticle scattered X-ray intensity in the heliosphere . . . . . . . . 128

3.11 Jupiter and comet Ikeya-Zhang X-ray spectrum . . . . . . . . . . . . . . 130

3.12 Total cross sections for X-ray scattering from cometary gas and dust . . . . 131

3.13 Average cometary X-ray spectrum due to scattering and fluorescence . . . . 132

3.14 Comet Ikeya-Zhang X-ray spectrum . . . . . . . . . . . . . . . . 134 


\section{LIST OF TABLES}

$2.1 \mathrm{H}+\mathrm{H}$ total cross sections analytic parameters . . . . . . . . . . . . 41

$2.2{ }^{4} \mathrm{He}+\mathrm{H}$ total cross sections analytic parameters . . . . . . . . . . . 41

$2.3{ }^{4} \mathrm{He}+{ }^{4} \mathrm{He}$ total cross sections analytic parameters . . . . . . . . . . . . 42

$2.4{ }^{3} \mathrm{He}+{ }^{3} \mathrm{He}$ total cross sections analytic parameters . . . . . . . . . . . . . 42

$2.5{ }^{4} \mathrm{He}+{ }^{3} \mathrm{He}$ total cross sections analytic parameters . . . . . . . . . . . . 43

$2.6{ }^{4} \mathrm{He}+\mathrm{O}$ total cross sections analytic parameters . . . . . . . . . . 43

2.7 Secondary hot atom $/$ molecule escape energies . . . . . . . . . . . . . 89

2.8 Secondary hot atom $/$ molecule probabilities and fluxes $\ldots \ldots \ldots 2$

2.9 Energetic neutral atom reflection probabilities . . . . . . . . . . . . . 94 


\section{Chapter 1}

\section{Introduction}

There has been great progress over the last several years in remote observation of astrophysical environments, both with Earth and spaced based telescopes, as well interplanetary satellites and rovers. The data obtained both remotely and in situ from these sources has allowed for a much greater understanding of our solar system, galaxy, and the universe beyond. Evolution of compositions and temperatures of the solar system planets and their moons, detection of large interstellar clouds encompassing enormous regions of the galaxy, and the detection of over 1000 exoplanets to date are all achievements which were attained through observation and modeling with accurate knowledge of fundamental atomic and molecular processes including scattering and interactions with radiation.

The scattering of atoms, molecules, and radiation are the most fundamental mechanisms of energy-momentum transfer in nature. Evolution of astrophysical gases and plasmas is governed by energy transfer and relaxation processes involving both radiation and particles. In particular, understanding the energy relaxation of fast $\mathrm{H}$ and $\mathrm{He}$ atoms, the most abundant gases in the universe [1], is crucial for an accurate description

of the physical environment. Helium is an important component of the interstellar gas 
as well as the upper atmospheres of planets and moons $[2,3]$. The interaction between neutral gas and space plasmas leads to the formation of energetic atomic particles. As a rule, nascent fast atoms are described by non-equilibrium energy distribution functions which differ significantly from the thermal Maxwellian distribution of a local gas [4-6]. Energies of the fast He atoms vary from $\mathrm{meV}$ to $\mathrm{keV}$ depending on the mechanism of their formation and parameters of the considered astrophysical object, but the criterion for the non-equilibrium distribution is very simple: the energy of a hot particle should be significantly larger than the thermal energies of ambient gas or plasma. This large energy range was investigated using quantum mechanical methods to develop accurate scattering parameters, used to model transport, thermalization, and momentum-energy transfer in several important astrophysical environments including the atmosphere of Mars and the local interstellar gas.

Scattering, absorption, and emission of photons are essential parts of the global energy relaxation in the universe. In this thesis, the scattering of X-rays by nanoparticles in different astrophysical environments was also investigated. X-rays may have extremely large transport lengths as cross sections with average gas and dust are small enough to consider most the X-rays collisionless in several systems. Nanoparticles, on the other hand, are efficient scatterers of X-rays as their geometrical size is comparable to that of the X-ray wavelengths. The interstellar dust, consisting mostly of nanometer size grains, is a major component of the interstellar medium which interacts with cosmic X-ray emission. Simple models were constructed to obtain scattering parameters for X-rays incident on different types of nanoparticles including carbon, silicon, and 
ice particles. These scattering parameters were utilized in simulations of different astrophysical environments including heliospheric dust clouds and cometary atmospheres. Obtained results were compared to available in situ data.

The layout of this work starts first with the scattering of atoms and molecules followed by the scattering of X-rays. Conclusions follow. 


\section{Chapter 2}

\section{Particle Scattering in Astrophysical Environments}

Energetic neutral atoms (ENAs), created through charge exchange (CX) collisions between energetic ions and neutral gases, are a great tool for remote imaging of space plasmas [7]. In addition to plasma imaging, ENAs are a major source of energy input into several astrophysical environments [8]. With the utilization of accurate, quantum mechanical CX cross sections, along with parameters of solar wind (SW) plasma, nascent ENA production rates have been calculated for the upper atmosphere of Mars, as well as the local interstellar cloud (LIC). Transport of ENAs in astrophysical environments was performed using Monte Carlo (MC) methods along with ab initio elastic cross sections which have been calculated for several atomic collisions up to $10 \mathrm{keV}$. Additionally, an empirical scaling procedure was constructed to predict collisional cross sections for very difficult atom-molecule and molecule-molecule collisions for which quantum, ab initio methods are not feasible. Properties of energy-momentum transfer were determined using accurate, anisotropic cross sections and compared to isotropic, hard sphere (HS) cross sections. Important parameters such as thermalization times, distances, and energy transferred to thermal gases were obtained for the atmosphere of Mars and the LIC. 


\subsection{Collisional Cross Sections}

Accurate energy-angular dependent cross sections are extremely useful in many branches of physics as they describe how energy-momentum is transferred from energetic regions of a system to less energetic regions, leading to an eventual thermalization. For the astrophysical systems of interest, specifically SW hydrogen and helium ions interacting with planetary and interstellar atmospheres, the energy range for nascent ENAs extends from hundreds eV/amu to several $\mathrm{keV} / \mathrm{amu}[9]$. While data on several theoretical and experimental cross sections exist within some intervals of this energy range, a complete database covering the entire energy range is lacking from the literature. Utilizing ab initio interaction potentials and quantum mechanical methods, accurate energy-angular cross sections have been calculated for collisions between atoms of major astrophysical gases $\mathrm{H}+\mathrm{H}, \mathrm{He}+\mathrm{H}, \mathrm{He}+\mathrm{He}$, and $\mathrm{He}+\mathrm{O}$ from temperatures $\sim 100 \mathrm{~K}$, to $10 \mathrm{keV}$ [10]. Additionally, empirical scaling cross sections have been developed for predictions of complicated atom-molecule and molecule-molecule collisions which are currently unrealistic for ab initio calculation at such high energies [11].

\subsubsection{Partial Wave Scattering}

Scattering, absorption, and emission of photons are essential parts of the global energy relaxation in the universe. In this thesis, collisions involving $\mathrm{H}+\mathrm{H}, \mathrm{He}+\mathrm{H}, \mathrm{He}+\mathrm{He}$, and $\mathrm{He}+\mathrm{O}$, utilized quantum mechanical partial wave $(\mathrm{PW})$ methods. Quantum scattering is often visualized for spherically symmetric potentials as an incoming, incident, plane wave in superposition with an outgoing, scattered, spherical wave. In the asymptotic 


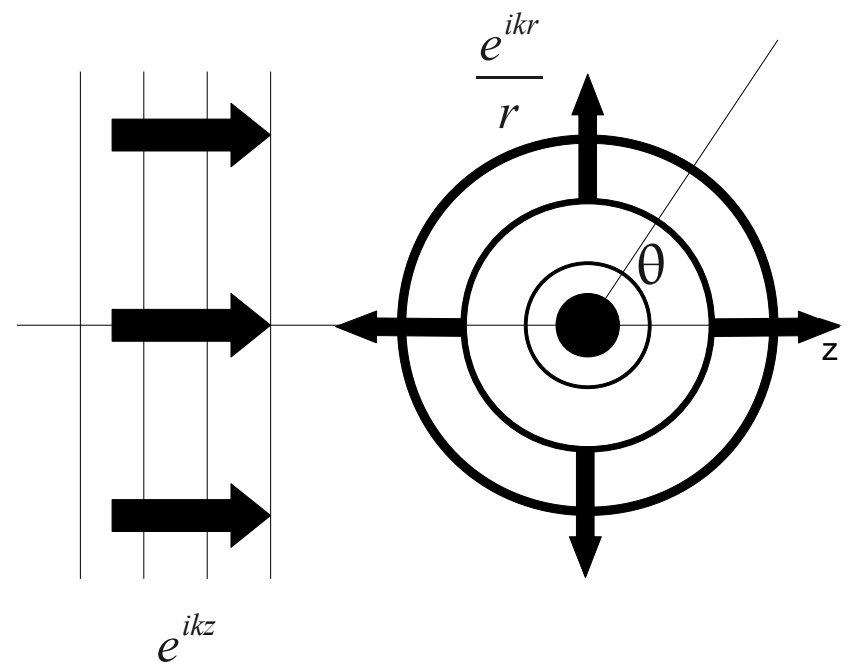

Fig. 2.1: Diagram of quantum mechanical partial wave scattering.

limit $(k r \gg 1)$ solutions to the Schrödinger equation take the form

$$
\psi(r, \theta) \approx A\left(e^{i k z}+f(\theta) \frac{e^{i k r}}{r}\right)=\psi_{i n}+\psi_{s c}
$$

where the incident wave $\psi_{\text {in }}$ is a plane wave in the $z$ direction and the scattered wave $\psi_{s c}$ has a complex scattering amplitude $f(\theta)$ in the direction given by the polar angle $\theta$ relative to the incident wave. Fig. 2.1 displays a diagram of the PW scattering process. The differential cross section is defined as the modulus squared of the complex scattering amplitude,

$$
\frac{d \sigma}{d \Omega}=|f(\theta)|^{2}
$$

while the total cross section is found by integrating the differential cross section over all solid angles

$$
\sigma=\int \frac{d \sigma}{d \Omega} d \Omega=\int|f(\theta)|^{2} d \Omega
$$


It is then the goal to determine the scattering amplitude $f(\theta)$ for a given interaction potential, the details of which follow.

The binary collision problem is best approached in the center of mass (CM) frame which allows for the Hamiltonian to be written for a single particle scattering from a potential field $V(r)$ as

$$
\hat{H}=-\frac{\hbar^{2}}{2 \mu} \nabla^{2}+V(r)
$$

where $\mu$ is the reduced mass of the system $\mu=\frac{m_{1} m_{2}}{m_{1}+m_{2}}$ and the corresponding energy of the system is found from applying the Hamiltonian to the wave function such that

$$
\hat{H} \psi=E \psi \text {. }
$$

The wave function, to which the Hamiltonian is applied, may be written as an infinite series such that

$$
\psi(r, \theta, \phi)=\sum_{l=0}^{\infty} \sum_{m=-l}^{l} C_{l m} R_{l k}(r) Y_{l}^{m}(\theta, \phi)
$$

where the constants $C_{l m}$ are to be determined and $k=\sqrt{2 \mu E / \hbar^{2}}$ is the wavenumber. The angular wavefunctions $Y_{l}^{m}(\theta, \phi)$ are the orthonormal set of spherical harmonics which form the solution to the spherical Schrödinger equation [12]. Defining $u_{l k}(r) \equiv$ $r R_{l k}(r)$ the radial Schrödinger equation for the spherically symmetric potential $V(r)$ becomes

$$
\frac{d^{2}}{d r^{2}} u_{l k}(r)+\left[k^{2}-\frac{l(l+1)}{r^{2}}-\frac{2 \mu}{\hbar^{2}} V(r)\right] u_{l k}(r)=0
$$

which shows both the interaction potential $U_{\text {int }}(r)=V(r)$ as well as the centrifugal potential $U_{c f}(r)=\frac{\hbar^{2}(l+1) l}{2 \mu r^{2}}$. 
The asymptotic solution to Eq. 2.7, when both $U_{\text {int }}(r)$ and $U_{c f}(r)$ may be neglected, takes the form

$$
\frac{d^{2}}{d r^{2}} u_{l k}(r)+k^{2} u_{l k}(r)=0
$$

and has the solution

$$
u_{l k}(r)=A_{k} e^{i k r}+B_{k} e^{-i k r}
$$

which is an outgoing spherical wave as required by Eq. 2.1, if $B_{k}=0$.

The radial Schrödinger equation may also be analyzed in the intermediate region, when the interaction potential $U_{\text {int }}(r)$ may be neglected but not the centrifugal potential $U_{c f}(r)$ due to the fact that the interaction potential $U_{\text {int }}(r)$ decays faster than $r^{-2}$. In these limits Eq. 2.7 becomes

$$
\frac{d^{2}}{d r^{2}} u_{l k}(r)+k^{2} u_{l k}(r)-\frac{l(l+1)}{r^{2}} u_{l k}(r)=0
$$

which has the solutions of linear combinations of spherical Bessel functions $j_{l}(x)$ and $n_{l}(x)$

$$
u_{l k}(r)=A_{l k} r j_{l}(k r)+B_{l k} r n_{l}(k r) .
$$

The first and second kinds of spherical Hankel functions are defined as linear combinations of spherical Bessel functions

$$
\begin{gathered}
h_{l}^{(1)}(x) \equiv j_{l}(x)+i n_{l}(x) \\
h_{l}^{(2)}(x) \equiv j_{l}(x)-i n_{l}(x) .
\end{gathered}
$$

It is informative to look at the asymptotic behaviors of the spherical Hankel functions to determine which type, first or second, should be used for the intermediate region 
wave function. In the limit that $x \rightarrow \infty$ the spherical Hankel functions become

$$
\begin{aligned}
& \lim _{x \rightarrow \infty} h_{l}^{(1)}(x)=(-i)^{l+1} \frac{e^{i x}}{x} \\
& \lim _{x \rightarrow \infty} h_{l}^{(2)}(x)=(i)^{l+1} \frac{e^{-i x}}{x}
\end{aligned}
$$

where the asymptotic limit of $h_{l}^{(1)}$ matches the expected asymptotic form of Eq. 2.1 so that the radial wave function in the intermediate region may be written as

$$
u_{l k}(r)=A_{l k} h_{l}^{(1)}(k r)
$$

Inserting the radial wave function, Eq. 2.14, into the generalized wave function, Eq. 2.6, results in the intermediate wave function of the form

$$
\psi(r, \theta, \phi)=A\left[e^{i k z}+\sum_{l, m} C_{l m} h_{l}^{(1)}(k r) Y_{l}^{m}(\theta, \phi)\right]
$$

The cylindrical symmetry of the scattering problem may be used to further simplify Eq. 2.15 since the potential is spherically symmetric and the incident wave is assumed to be a plane wave moving in the $\hat{z}$ direction. The incident wave does not contain any net angular momentum in the problem formulation and thus the scattered wave must also contain no net angular momentum due to conservation laws. The spherical harmonics are then reduced to have only $m=0$ values so that

$$
Y_{l}^{m}(\theta, \phi) \rightarrow Y_{l}^{0}(\theta, \phi)=\sqrt{\frac{2 l+1}{4 \pi}} P_{l}(\cos \theta)
$$

where $P_{l}(\cos \theta)$ are the set of Legendre polynomials. If the constants $C_{l m}$ are rewritten as

$$
C_{l} \equiv i^{l+1} k \sqrt{4 \pi(2 l+1)} a_{l}
$$


with $a_{l}$ being defined as the $l^{\text {th }}$ partial wave scattering amplitude, the intermediate wave function takes the form

$$
\psi(r, \theta)=A\left[e^{i k z}+k \sum_{l=0}^{\infty} i^{l+1}(2 l+1) a_{l} h_{l}^{(1)}(k r) P_{l}(\cos \theta)\right]
$$

Comparing Eq. 2.18 with our original asymptotic wave function Eq. 2.1 and using the limiting case of the spherical Hankel function Eq. 2.13 it is easy to write the scattering amplitude $f(\theta)$ as a function of partial wave amplitudes $a_{l}$ as

$$
f(\theta)=\sum_{l=0}^{\infty}(2 l+1) a_{l} P_{l}(\cos \theta)
$$

It is now our goal to determine partial wave amplitudes $a_{l}$ for all $l$ partial waves to obtain the total scattering amplitude.

It is often easier to work with phase shifts $\delta_{l}$ which are real numbers as opposed to partial wave amplitudes $a_{l}$ which are complex. This may be done by first writing the incident plane wave as an infinite series of spherical waves using the Rayleigh formula as

$$
e^{i k z}=\sum_{l=0}^{\infty} i^{l}(2 l+1) j_{l}(k r) P_{l}(\cos \theta)
$$

where as before, $j_{l}(k r)$ and $P_{l}(\cos \theta)$ are the spherical Bessel functions of the first kind and the Legendre polynomials respectively. Inserting Eq. 2.20 into Eq. 2.18 results in an intermediate region wave function described entirely by an infinite sum as

$$
\psi(r, \theta)=A \sum_{l=0}^{\infty} i^{l}(2 l+1)\left[j_{l}(k r)+i k a_{l} h_{l}^{(1)}(k r)\right] P_{l}(\cos \theta) .
$$

To obtain expressions for scattering phase shifts, it is easiest to work within approximating limits. Assuming there is no interaction potential at all, $V(r)=0$, then all partial 
wave amplitudes should be zero, $a_{l}=0$, for all values of $l$. In this limit of $V(r)=0$, the wave function for the $l^{\text {th }}$ partial wave from Eq. 2.21 can be written as

$$
\psi_{V=0}^{(l)}=A i^{l}(2 l+1) j_{l}(k r) P_{l}(\cos \theta) .
$$

Defining the spherical Bessel function as a linear combination of spherical Hankel functions, Eq. 2.12, and utilizing the asymptotic limits of the spherical Hankel functions, Eq. 2.13, gives the asymptotic limit of $j_{l}(k r)$

$$
\begin{array}{r}
j_{l}(k r)=\frac{1}{2}\left[h_{l}^{(1)}(k r)+h_{l}^{(2)}(k r)\right] \\
\lim _{k r \rightarrow \infty} j_{l}(k r)=\frac{1}{2 k r}\left[(-1)^{l+1} e^{i k r}+i^{l+1} e^{-i k r}\right] .
\end{array}
$$

The asymptotic wave function for no interaction potential can then be written by applying Eq. 2.23 to Eq. 2.22

$$
\psi_{V=0}^{(l)}=A \frac{2 l+1}{2 i k r}\left[e^{i k r}-(-1)^{l} e^{-i k r}\right] P_{l}(\cos \theta)
$$

which is a linear combination of incoming, $e^{-i k r}$, and outgoing, $e^{i k r}$, spherical waves, with angular modulation dependent on the Legendre polynomials. Eq. 2.24 is very illuminating as it breaks down the scattered wave function in terms of spherical waves and allows us to directly write the scattered wave function in the presence of an interaction potential $V(r) \neq 0$ by simply adding an accumulated phase, $2 \delta_{l}$, to the outgoing spherical wave

$$
\psi^{(l)}=A \frac{2 l+1}{2 i k r}\left[e^{i k r+2 \delta_{l}}-(-1)^{l} e^{-i k r}\right] P_{l}(\cos \theta) .
$$

The asymptotic wave function in Eq. 2.25, which is a function of phase shifts $\delta_{l}$, can now be directly compared with the asymptotic wave function in Eq. 2.21, which is a function 
of complex partial wave amplitudes $a_{l}$, so that a direct relationship can be established

$$
a_{l}=\frac{1}{2 i k}\left(e^{2 i \delta_{l}}-1\right)=\frac{1}{k} e^{i \delta_{l}} \sin \delta_{l} .
$$

Using the new relationship in Eq. 2.26 in addition to Eq. 2.19 results in the scattering amplitude as a function of phase shifts

$$
f(\theta)=\frac{1}{k} \sum_{l=0}^{\infty}(2 l+1) e^{i \delta_{l}} \sin \delta_{l} P_{l}(\cos \theta)
$$

which, using Eq. 2.2 leads to the differential cross sections being defined in terms of the $l^{\text {th }}$ partial wave phase shifts as

$$
\frac{d \sigma}{d \Omega}=|f(\theta)|^{2}=\frac{1}{k^{2}} \sum_{l, l^{\prime}}(2 l+1)\left(2 l^{\prime}+1\right) e^{i\left(\delta_{l}-\delta_{l^{\prime}}\right)} \sin \delta_{l} \sin \delta_{l^{\prime}} P_{l}(\cos \theta) P_{l^{\prime}}(\cos \theta) .
$$

Integrating the differential cross sections over all solid angle give the total scattering cross section, Eq. 2.3, which takes the form

$$
\sigma=\frac{2 \pi}{k^{2}} \sum_{l, l^{\prime}}(2 l+1)\left(2 l^{\prime}+1\right) e^{i\left(\delta_{l}-\delta_{l^{\prime}}\right)} \sin \delta_{l} \sin \delta_{l^{\prime}} \int_{0}^{\pi} P_{l}(\cos \theta) P_{l^{\prime}}(\cos \theta) \sin \theta d \theta .
$$

Utilizing the orthogonality condition of the Legendre polynomials

$$
\int_{0}^{\pi} P_{l}(\cos \theta) P_{l^{\prime}}(\cos \theta) \sin \theta d \theta=\frac{2}{2 l+1} \delta_{l, l^{\prime}}
$$

the only non-zero solution is when $l=l^{\prime}$ so the total cross section in Eq. 2.29 becomes

$$
\sigma=\frac{4 \pi}{k^{2}} \sum_{l=0}^{\infty}(2 l+1) \sin ^{2} \delta_{l}=\frac{4 \pi}{k} \operatorname{Im}[f(\theta=0)]
$$

which is a famous result referred to as the optical theorem [12].

Collisions involving identical particles require extra treatment using quantum mechanical methods as the projectile and the target are indistinguishable in the center 
of mass frame. For identical particle collisions, Eq. 2.1 is re-written as

$$
\psi(r, \theta) \approx e^{i k z} \pm e^{-i k z}+[f(\theta) \pm f(\pi-\theta)] \frac{e^{i k r}}{r},
$$

which describes two incoming plane waves and scattering amplitudes for scattering at an angle $\theta$ and $\pi-\theta$ [13]. If the particles have integer spin, bosons, the collision is symmetric and the $(+)$ term in Eq. 2.32 is used. For half integer spin, fermions, the antisymmetric (-) term is used in Eq. 2.32. This symmetry leads to a total scattering amplitude defined as

$$
F_{ \pm}(\theta)=f(\theta) \pm f(\pi-\theta)=\frac{1}{k} \sum_{l}(2 l+1) e^{i \delta_{l}} \sin \delta_{l} P_{l}(\cos \theta)\left[1 \pm(-1)^{l}\right]
$$

and total cross section

$$
\sigma=\frac{8 \pi}{k^{2}} \sum_{l}(2 l+1) \sin ^{2} \delta_{l}\left[1 \pm\left(-1^{l}\right)\right]=\frac{8 \pi}{k} \operatorname{Im}\left[F_{ \pm}(\theta=0)\right]
$$

for both symmetric and antisymmetric collisions. The appropriate identical particle treatments were utilized for collisions in astrophysical environments including $\mathrm{H}+\mathrm{H}$, ${ }^{3} \mathrm{He}+{ }^{3} \mathrm{He}$, and ${ }^{4} \mathrm{He}+{ }^{4} \mathrm{He}$.

With the derivation of Eq. 2.28, 2.31, 2.33, and 2.34 it is only a matter of determining the partial wave phase shifts $\delta_{l}$ for a given interaction potential to obtain differential and total cross sections. Phase shifts were found numerically using $\mathrm{Nu}-$ merov's method [14]. Numerov's method is suited for numerically solving differential equations of the form

$$
\left(\frac{d^{2}}{d x^{2}}+f(x)\right) y(x)=0
$$


which has the exact form as our radial Schrödinger equation seen in Eq. 2.7 with

$$
\begin{array}{r}
f(x) \rightarrow\left[k^{2}-\frac{l(l+1)}{r^{2}}-\frac{2 \mu}{\hbar^{2}} V(r)\right], \\
y(x) \rightarrow u_{l k}(r) .
\end{array}
$$

Using a discrete, 1D grid with a step size of $h$, Numerov's method yields a solution for the $y_{n+1}$ grid given knowledge of $y_{n}$ and $y_{n-1}$ such that

$$
y_{n+1}=\frac{\left(2-C_{1} h^{2} f_{n}\right) y_{n}-\left(1+C_{2} h^{2} f_{n-1}\right) y_{n-1}}{1+C_{2} h^{2} f_{n+1}}+O\left(h^{6}\right)
$$

and where the constants $C_{1}$ and $C_{2}$ are $\frac{5}{6}$ and $\frac{1}{12}$ respectively, and the potential terms $f_{n}$ are known for the entire grid as they are only a function of energy, $k$, partial wave number, $l$, and interaction potential, $V(r)$. Numerov's method is a $4^{\text {th }}$ order method so that the neglected terms are of order $h^{6}$ [14]. For a given collision energy, E, Eq. 2.37 was solved starting at an interaction distance, $r_{0}$, which had a potential energy such that $V\left(r_{0}\right) \gg E$ so that the wave function could be assumed to be zero, $\psi\left(r_{0}\right) \approx 0$. This allows for the initial wave function propagation conditions $y_{0}=0$ and $y_{1}=0.01$ where the value of $y_{1}$ is a small seed value to begin propagation of the wave function. Once the wave function is propagated to a distance in which $k r \gg 1$ holds, the derivative of the wave function is calculated numerically and compared with the derivative of the asymptotic wave function solution in the case of no interaction potential, Eq. 2.24. This yields the phase shift for the $l^{\text {th }}$ partial wave. The procedure described above is repeated for increasing partial wave numbers until the phase shifts converge to a value where $\left|\delta_{l}\right|<10^{-9}$ which is the numerically significant limit of phase shifts to the differential and total cross sections. Fig. 2.2 displays the number of partial waves required for convergence of the scattering amplitude for the quantum collisions considered, consisting 


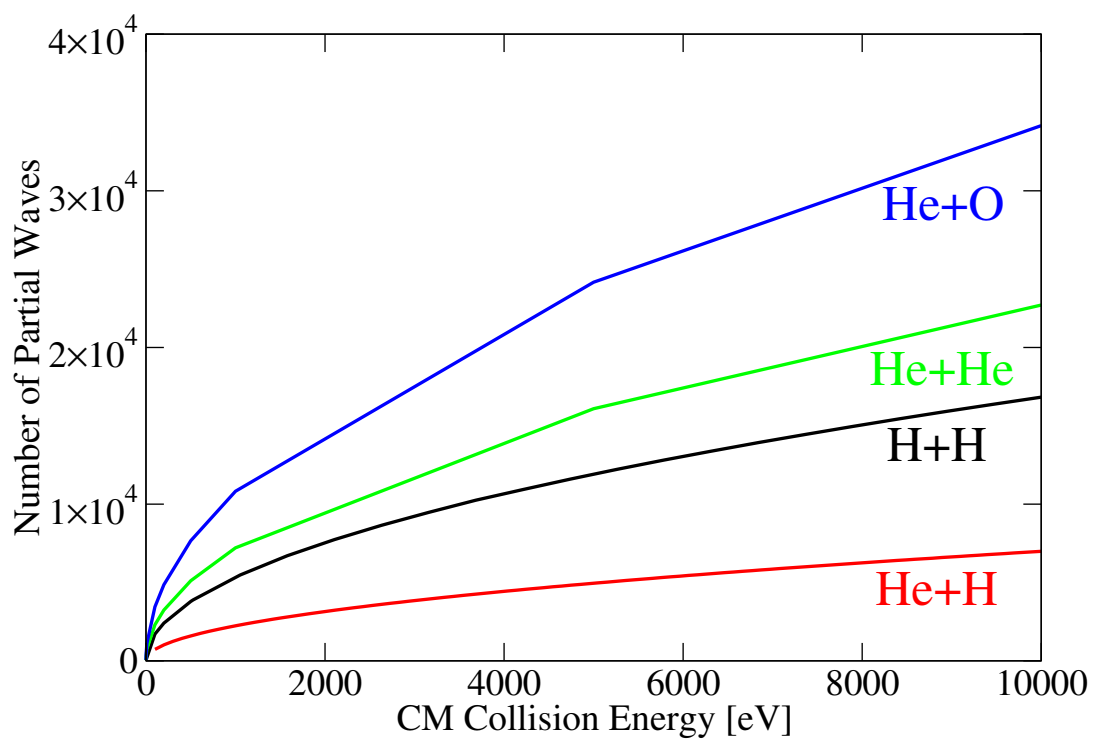

Fig. 2.2: Number of partial wave required for convergence of the scattering amplitude as a function of the center of mass collision energy for collisions of $\mathrm{H}+\mathrm{H}$, $\mathrm{He}+\mathrm{H}, \mathrm{He}+\mathrm{He}$, and $\mathrm{He}+\mathrm{O}$.

of $\mathrm{H}+\mathrm{H}, \mathrm{He}+\mathrm{H}, \mathrm{He}+\mathrm{He}$, and $\mathrm{He}+\mathrm{O}$ as a function of collision energy. The number of partial waves required for convergence is sensitive to both the reduced mass and the long range component of the interaction potentials.

Software was constructed to solve for the scattering phase shifts for a given interaction potential, Eq. 2.37, for several different partial waves simultaneously utilizing the message passing interface (MPI) [15]. Communication between the processors was minimized to allow for the best possible parallel performance. The parallel speedup may be objectively analyzed through use of parallel code timers to determine how the solution time decreases as the number of processors increases. Defining the parallel 
speedup as

$$
S_{n}=\frac{\Delta t_{1}}{\Delta t_{n}}
$$

where $\Delta t_{1}$ is the time taken to solve the problem with one processor and $\Delta t_{n}$ is the time taken to solve the same problem with $n$ processors. In the theoretical ideal case the speedup is linear with the number of processors so that $S_{n}=n$. The speedup was measured for the parallel phase shift software using a desktop cluster consisting of dual hex-core, hyper-threaded Intel Xeon X5650 2.67GHz with a total of $96 \mathrm{~GB}$ of memory (Firsov) at the University of Connecticut as well as with several nodes of the Cray XT5 supercomputer with dual hex-core AMD Opteron 2435 processors and 16 GB of memory per node (Jaguar) at Oak Ridge National Laboratory. Fig. 2.3 shows the speedup achieved on each architecture as well as the theoretical ideal case of linear speedup for collisions of $\mathrm{He}+\mathrm{O}$ at collision energies ranging from $10^{-2} \mathrm{eV}$ to $2 \mathrm{keV}$. The Firsov architecture maintained near linear speedup for all collision energies up to 12 processors when the speedup slope flattens out drastically as seen in Fig. 2.3. This change in parallel performance at 12 processors is due to the hyper-threading technology of the Intel Xeon X5650 which mimics 24 processors with individual address spaces, but lack of physical cores leads to memory bottlenecks and reduced performance. The speedup observed on the Jaguar architecture, on the other hand, has near ideal speedup up to 36 processors for all collision energies of $1 \mathrm{eV}$ or higher. The poor performance of the smaller collision energies is a result of large communication overhead relative to the problem difficulty. This makes the use of several processors on a larger cluster much more efficient for harder collision problems which require several thousand partial waves 
for convergence, Fig. 2.2.

\subsubsection{Interaction Potentials}

The accuracy and quality of the calculated cross sections are heavily dependent on the interaction potential utilized in solving Eq. 2.4. For all partial wave calculations $a b$ initio $\mathrm{H}+\mathrm{H}, \mathrm{He}+\mathrm{H}, \mathrm{He}+\mathrm{He}$, and $\mathrm{He}+\mathrm{O}$ potentials were used to determine collisional cross sections. The interaction potential for $\mathrm{He}+\mathrm{He}$ was used for a description of helium collisions and its isotopes ${ }^{4} \mathrm{He}+{ }^{4} \mathrm{He},{ }^{3} \mathrm{He}+{ }^{3} \mathrm{He}$, and ${ }^{4} \mathrm{He}+{ }^{3} \mathrm{He}$, changing the reduced mass as needed for different isotopes [16]. Collisions of $\mathrm{He}+\mathrm{H}$ utilized interaction potentials constructed using multi-reference configuration interaction [17] and coupled cluster [18] methods, with the final potential being further extrapolated to the complete basis set limit [19] using the aug-cc-pv5z basis set [20]. Multi-channel calculations were needed for $\mathrm{H}+\mathrm{H}$ and $\mathrm{He}+\mathrm{O}$ collisions which include singlet, $\sigma$, and triplet, $\pi$, interaction potentials. For these collisions, cross sections were computed for the individual scattering channels and then combined using statistical branching ratios to determine differential and total scattering cross sections. The potentials employed in the calculations for $\mathrm{He}+\mathrm{O}$ were computed using the same methods as for $\mathrm{He}+\mathrm{H}$ and with branching ratios of $2 / 3$ and $1 / 3$ for the $\pi$ and $\sigma$ states [21]. Collisions of $\mathrm{H}+\mathrm{H}$ utilized the interaction potentials provided by [22] for both $\pi$ and $\sigma$ states with branching ratios of $1 / 4$ and $3 / 4$ respectively [13]. Fig. 2.4 displays the interaction potentials for all collision species.

For high energy scattering the colliding particles may reach very small interparticle distances and the interaction potentials described above had to be updated with core potentials which were used for small interaction distances. This was accomplished 
Firsov
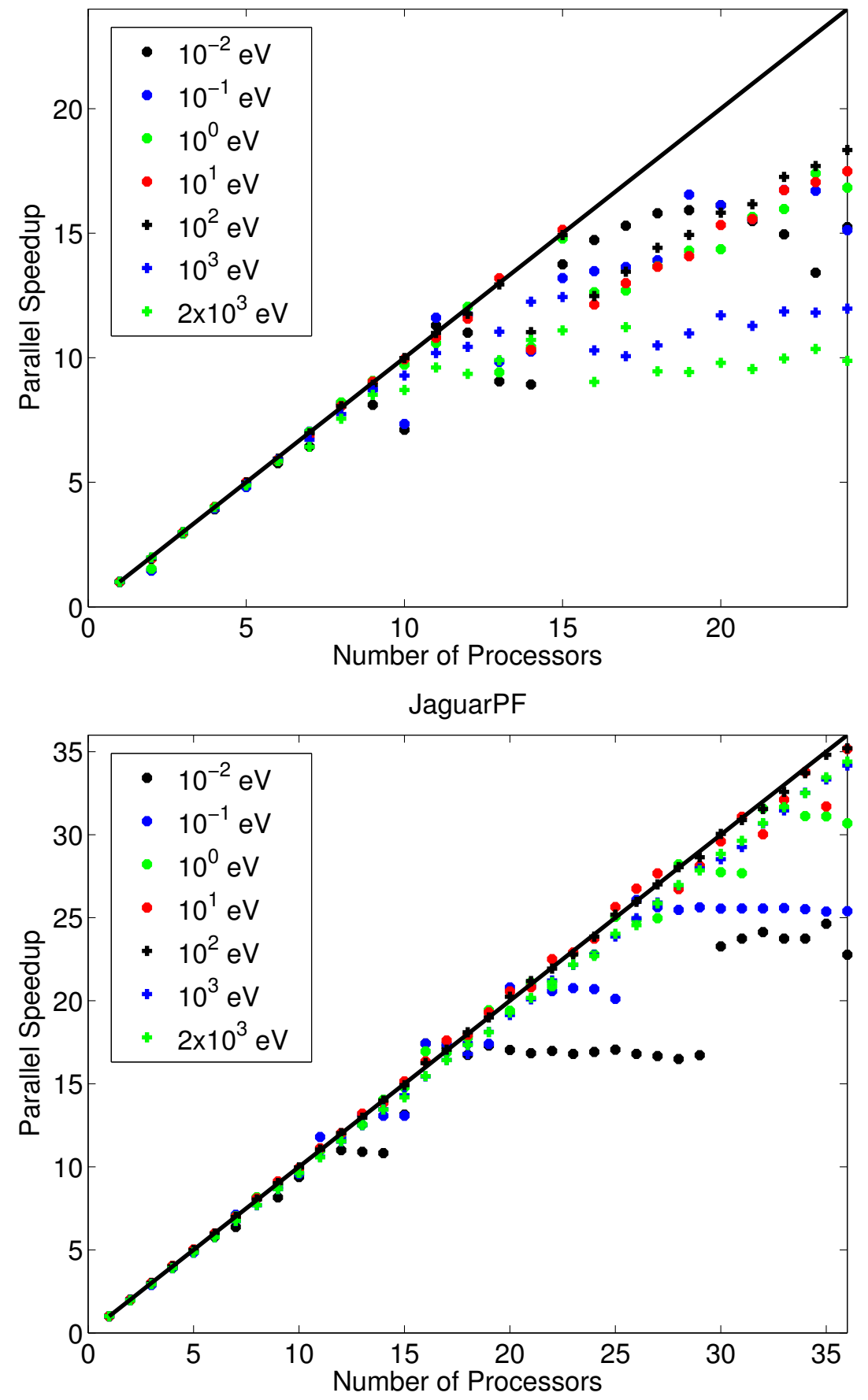

Fig. 2.3: Parallel speedup for solutions to the collision of $\mathrm{He}+\mathrm{O}$ for several collision energies on both the Firsov and Jaguar architectures. Ideal speedup is shown as a black solid line in each figure. 


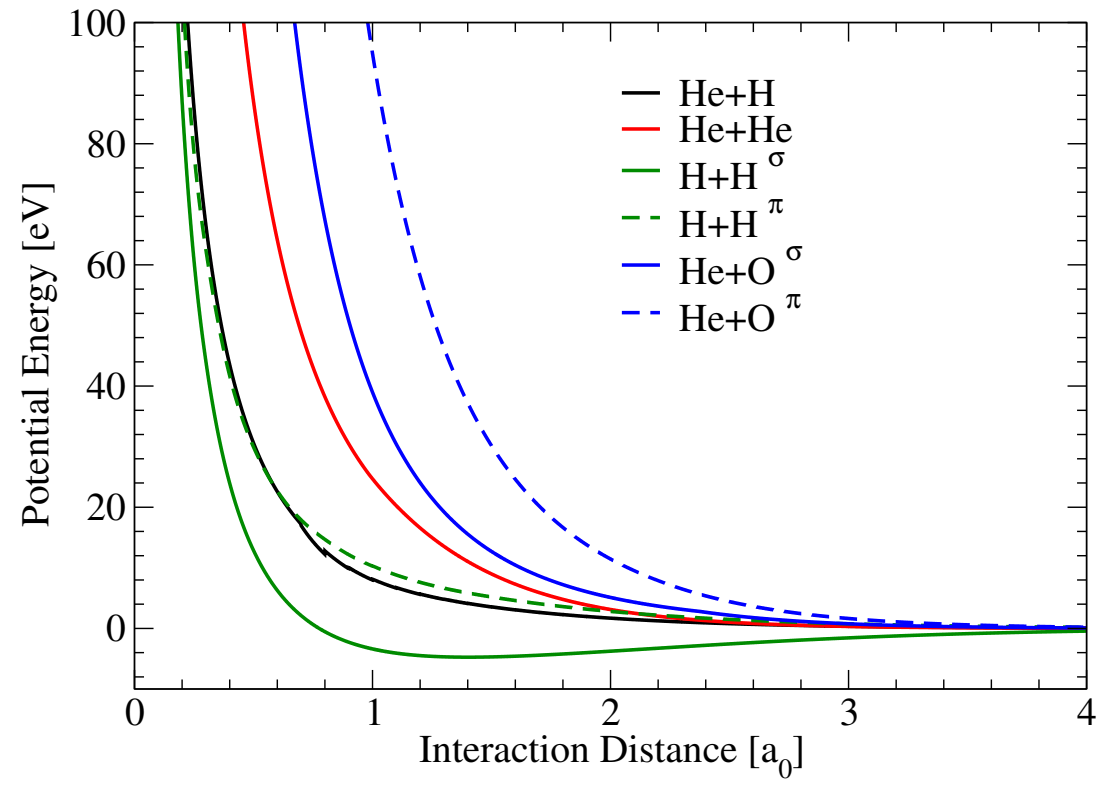

Fig. 2.4: Ab initio interaction potentials for $\mathrm{H}+\mathrm{H}, \mathrm{He}+\mathrm{He}, \mathrm{He}+\mathrm{H}$, and $\mathrm{He}+\mathrm{O}$. Singlet, $\sigma$, and triplet, $\pi$, states are shown for collisions of $\mathrm{H}+\mathrm{H}$ and $\mathrm{He}+\mathrm{O}$ which require multi-channel calculations. 
utilizing a screening core potential [23] of the form

$$
V(r)=\frac{Z_{1} Z_{2} e^{2}}{r} \phi\left(\frac{r}{a}\right)
$$

where $Z_{1}$ and $Z_{2}$ are the number of electrons present in the projectile and target and $\phi(r / a)$ is an empirical screening function defined as

$$
\phi(x)=\sum_{i=1}^{4} \alpha_{i} \exp \left(-\beta_{i} x\right) .
$$

The constants $\alpha_{i}$ are $0.1818,0.5099,0.2802$, and 0.02817 while the constants $\beta_{i}$ are 3.2 , 0.9423, 0.4029, and 0.2016 for $i=1-4$ respectively [24]. The length factor $a$ in Eq. 2.39 is the screening length and has the form

$$
a=\frac{0.88534 a_{0}}{Z_{1}^{0.23}+Z_{2}^{0.23}}
$$

where $a_{0}$ is the Bohr radius [23]. The potentials in Eq. 2.39 were further modified by multiplicative and additive constants to make them continuous with ab initio potentials and continuous in the first and second derivatives. With use of these modified interaction potentials with the partial wave methods described above, calculation of high energy scattering cross sections is possible.

\subsubsection{Partial Wave Cross Sections}

Using the quantum mechanical partial wave methods of Section 2.1.1 in addition to the interaction potentials of Section 2.1.2 allows for numeric calculation of both differential and total cross sections. The cross section calculations provide complete coverage over the energy interval from $10 \mathrm{meV}$ to $10 \mathrm{keV}$. This range of energies is important for several astrophysical and atmospheric applications as it also covers the velocity spectrum of 


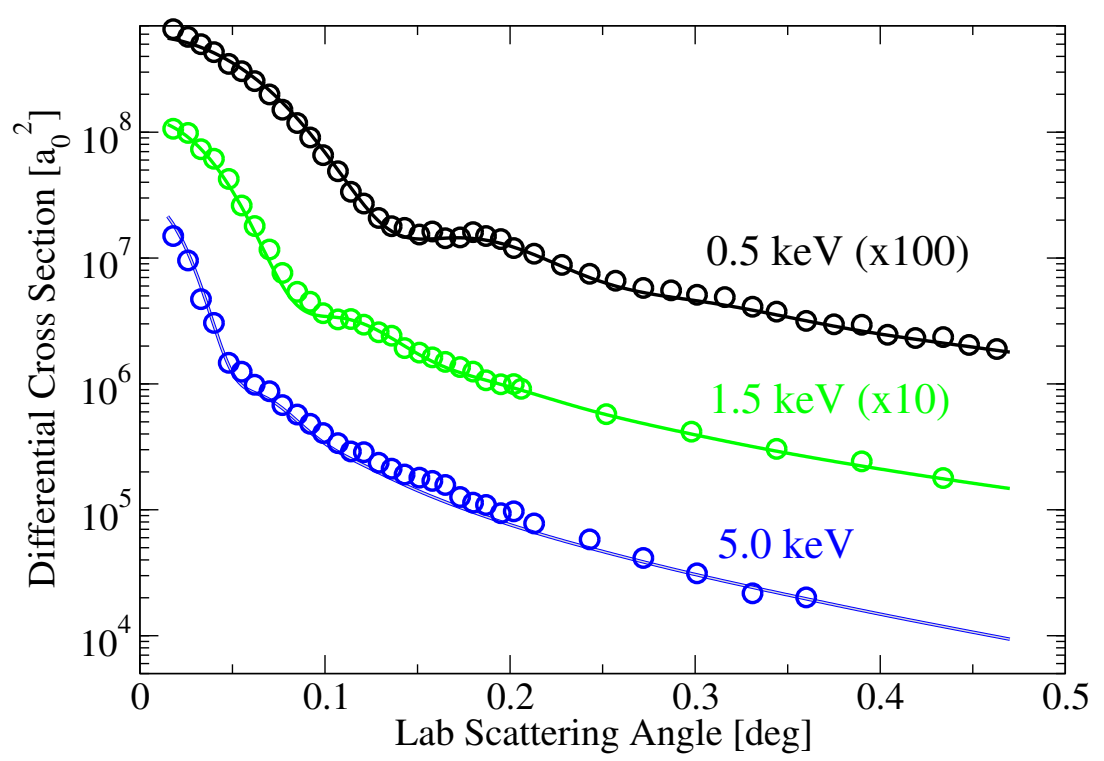

Fig. 2.5: Partial wave differential cross sections for ${ }^{4} \mathrm{He}+{ }^{4} \mathrm{He}$ collisions compared to experimental scattering data, shown as circles, for three different collision energies. The collision energies, scattering angles, and differential cross sections are all shown in the laboratory frame.

SW ions, which in turn produce ENAs [10]. Details of the calculated cross sections, comparisons with laboratory data, and development of analytic fitting formulas follow.

Differential cross sections for ${ }^{4} \mathrm{He}+{ }^{4} \mathrm{He}$ were calculated and compared to experimental data [25] for three different mid-to-high range energies at small laboratory scattering angles, less than $0.5^{\circ}$, with good agreement as seen in Fig. 2.5. Comparisons between partial wave $\mathrm{He}+\mathrm{O}$ differential cross sections and experimental scattering results are shown in Fig. 2.6 with one set of experimental data shown as plus signs [26] and the other shown as circles [27]. Fig. 2.6 also displays partial wave differential cross sections for a low energy, $1 \mathrm{eV}$ collision to compare how drastically different the differential 


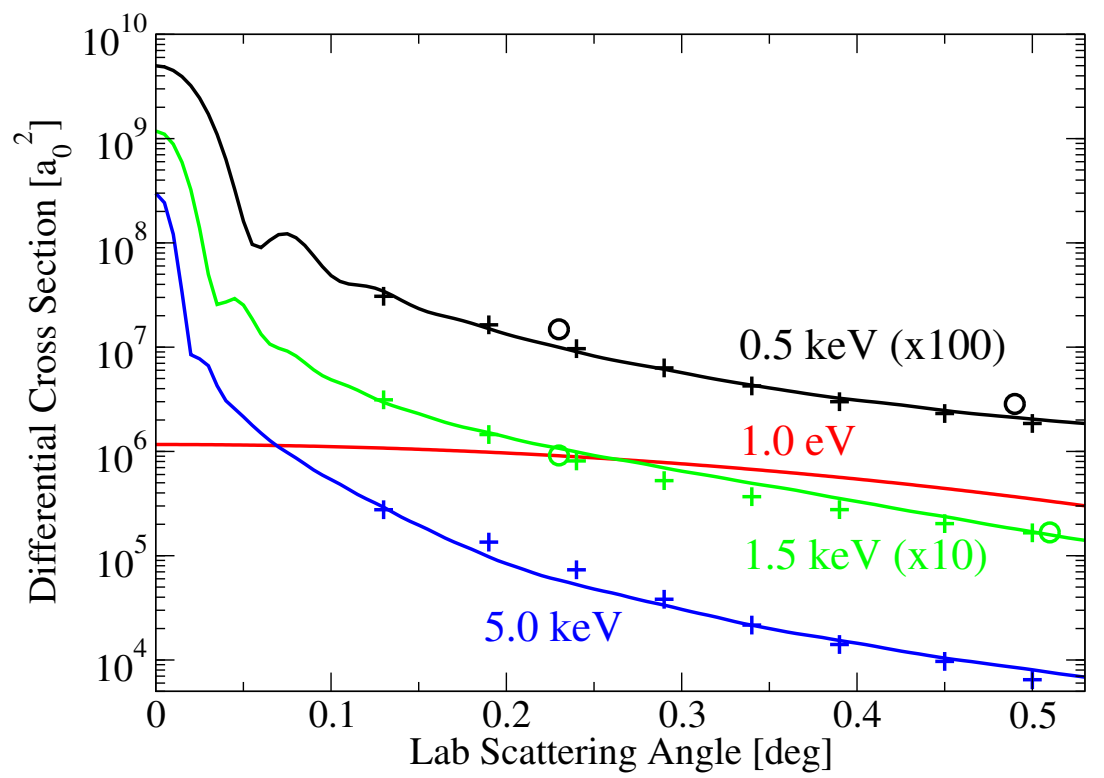

Fig. 2.6: Partial wave differential cross sections for $\mathrm{He}+\mathrm{O}$ collisions compared to experimental scattering data, shown as circles and pluses, for three different collision energies. Additionally, the partial wave differential cross section for a low energy, $1 \mathrm{eV}$, collision is shown for comparison with higher energies. The collision energies, scattering angles, and differential cross sections are all shown in the laboratory frame. 


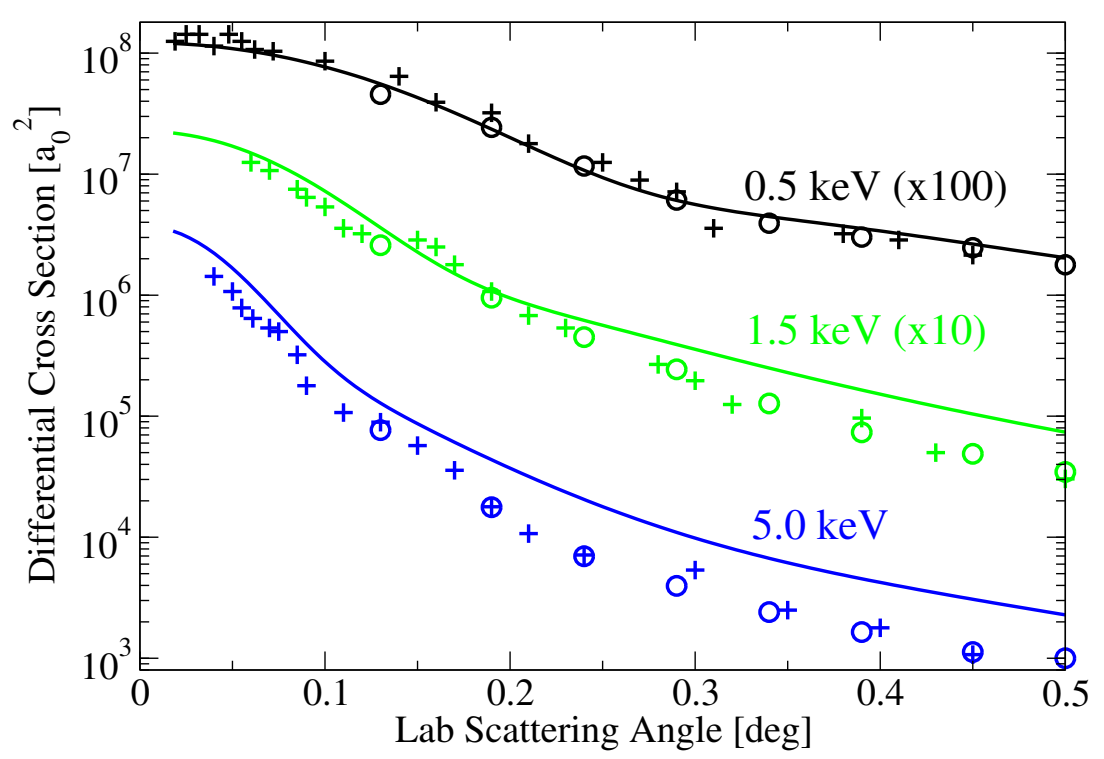

Fig. 2.7: Partial wave differential cross sections for $\mathrm{He}+\mathrm{H}$ collisions compared to experimental scattering data, shown as circles and pluses, for three different collision energies. The collision energies, scattering angles, and differential cross sections are all shown in the laboratory frame.

cross sections are with different energy scales. In particular, the $1 \mathrm{eV}$ collision differential cross sections in Fig. 2.6 show how extremely forward peaked the cross sections become with increasing energy by comparing to the $0.5,1.5$, and $5 \mathrm{keV}$ differential cross sections. Partial wave differential cross sections for collisions of $\mathrm{He}+\mathrm{H}$, and comparisons to experimental data are shown in Fig. 2.7. Two different sets of experimental data are shown in Fig. 2.7 with one set shown as plus signs [28] and the other shown as circles [29]. Expected differences between experimental and theoretical large-angle differential cross sections were found for $\mathrm{He}+\mathrm{O}$ and $\mathrm{He}+\mathrm{H}$ collisions in the partial wave calculations and were also previously seen in the literature [30]. Large-angle (small impact param- 


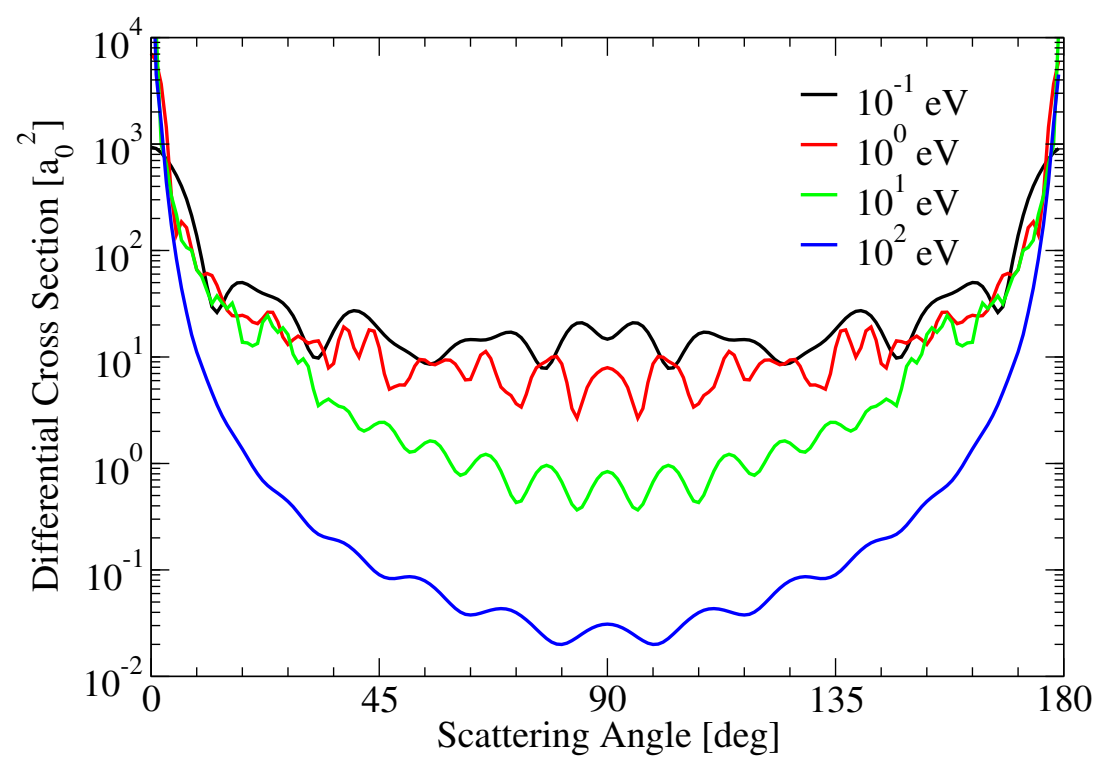

Fig. 2.8: Partial wave differential cross sections for $\mathrm{H}+\mathrm{H}$ collisions. The collision energies, scattering angles, and differential cross sections are all shown in the center of mass frame.

eter) scattering of atomic particles at high collision energies requires consideration of inelastic scattering channels even for cases when the total elastic cross section is significantly larger than inelastic ones. Experimental differential cross sections for collisions of $\mathrm{H}+\mathrm{H}$ are not available in the literature due to the difficulty associated with keeping individual target hydrogen atoms from binding into $\mathrm{H}_{2}$ dimers. In lieu of experimental data, comparisons with previous theoretical differential cross sections for collisions of $\mathrm{H}+\mathrm{H}$ up to $100 \mathrm{eV}$ [31] have been used to confirm the validity of calculated cross sections. Fig. 2.8 displays differential cross sections for four energies which have all been compared with good agreement to previous theoretical calculations [31]. The identical particle symmetry, Eq. 2.33, can be seen in Fig. 2.8 about the scattering angle of $\pi / 2$ 


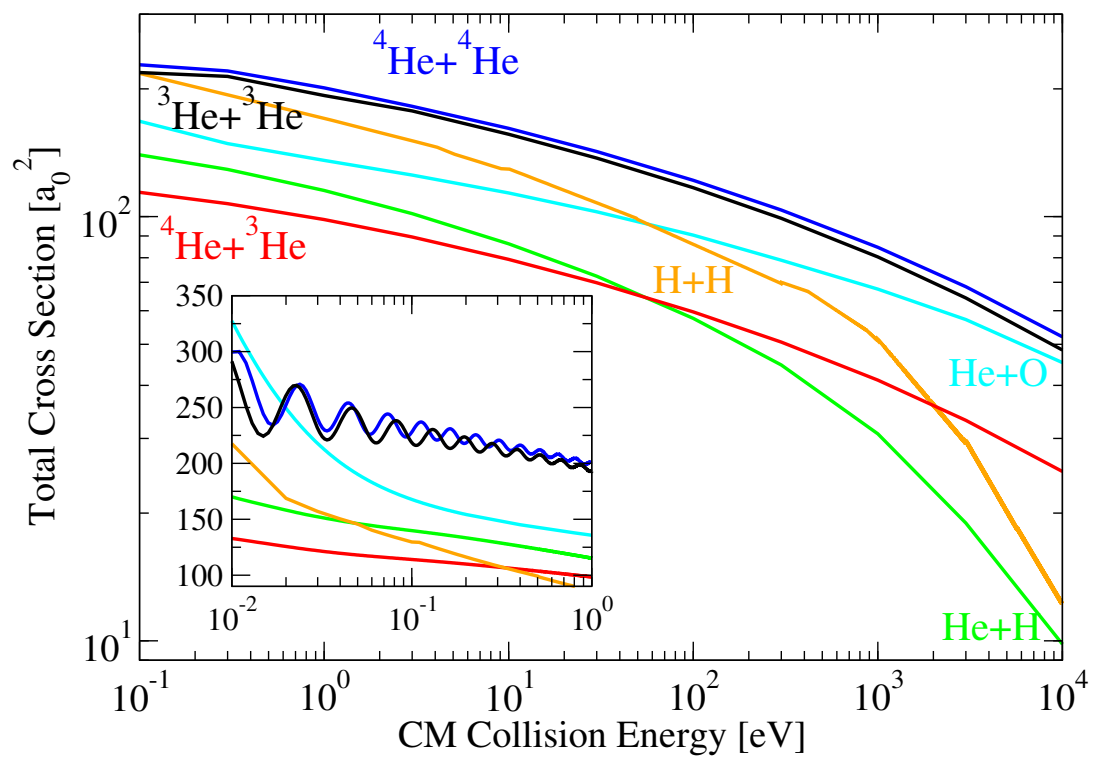

Fig. 2.9: Partial wave total cross sections for all collisions species. Inset displays low energy total cross sections. The collision energies are all shown in the center of mass frame.

due to the indistinguishability of the projectile and target atoms. Unfortunately no differential cross sections, either theoretical or experimental, are available in the literature for comparison with our results for energies higher than $100 \mathrm{eV}$.

In addition to differential cross sections, total cross sections were also obtained using the optical theorems of Eqs. 2.31 and 2.34. Fig. 2.9 displays the partial wave total cross sections from $10 \mathrm{meV}$ to $10 \mathrm{keV}$, effectively covering 6 orders of magnitude in energy. An analytic database was developed to easily distribute these accurate total cross sections using simple interpolation formulas. The partial wave cross sections were fit to the analytic form

$$
\sigma(E)=\sigma_{0}\left(\frac{E_{0}}{E}\right)^{\alpha}
$$


where the constants $\sigma_{0}$ and $\alpha$ were fit over several different energy intervals and the constant $E_{0}$ was equal to $1 \mathrm{keV}$ for all energies [10]. Tables 2.1 to 2.6 give the values for the fitting parameters $\sigma_{0}$ and $\alpha$ over the entire energy interval.

With accurate knowledge of both differential and total cross sections, a normalized probability density distribution of scattering angles may be defined as

$$
\rho(E, \theta)=\frac{2 \pi \sin \theta}{\sigma(E)}|f(E, \theta)|^{2} .
$$

Fig. 2.10 displays 2D heat maps of the probability density distribution of Eq. 2.43 as a function of both scattering angle $\theta$ and collision energy $E$ for all non-identical collision species. The "hottest" spots in Fig. 2.10 indicate the most probable scattering angles for a given collision energy, showing how extremely forward peaked the cross sections are for all energies higher than $\sim 1 \mathrm{eV}$. Additionally, using the normalization property of Eq. 2.43 the cumulative probability of scattering angles, which gives a probability of the value of the scattering angle to be smaller than a given angle $\theta$, is defined as

$$
P(E, \theta)=\int_{0}^{\theta} \rho\left(E, \theta^{\prime}\right) d \theta^{\prime}
$$

where $P(E, \theta) \in[0,1]$. Analysis of Eq. 2.44 gives deep insight into how drastically the range of contributing scattering angles changes with collision energy in a normalized fashion. For example, the experimental values for the integrated cross sections of the $\mathrm{He}+\mathrm{H}$ collision have been reported with minimal laboratory detection angle $\theta_{m}$ values of $0.018^{\circ}, 0.05^{\circ}$, and $0.13^{\circ}[29,32,33]$. Particles with scattering angles less than $\theta_{m}$ had not been detected. Fig. 2.11 shows the partial wave cumulative distributions as a function of scattering angles for collisions of $\mathrm{He}+\mathrm{H}$ and for laboratory frame collision energies of 0.5 , 

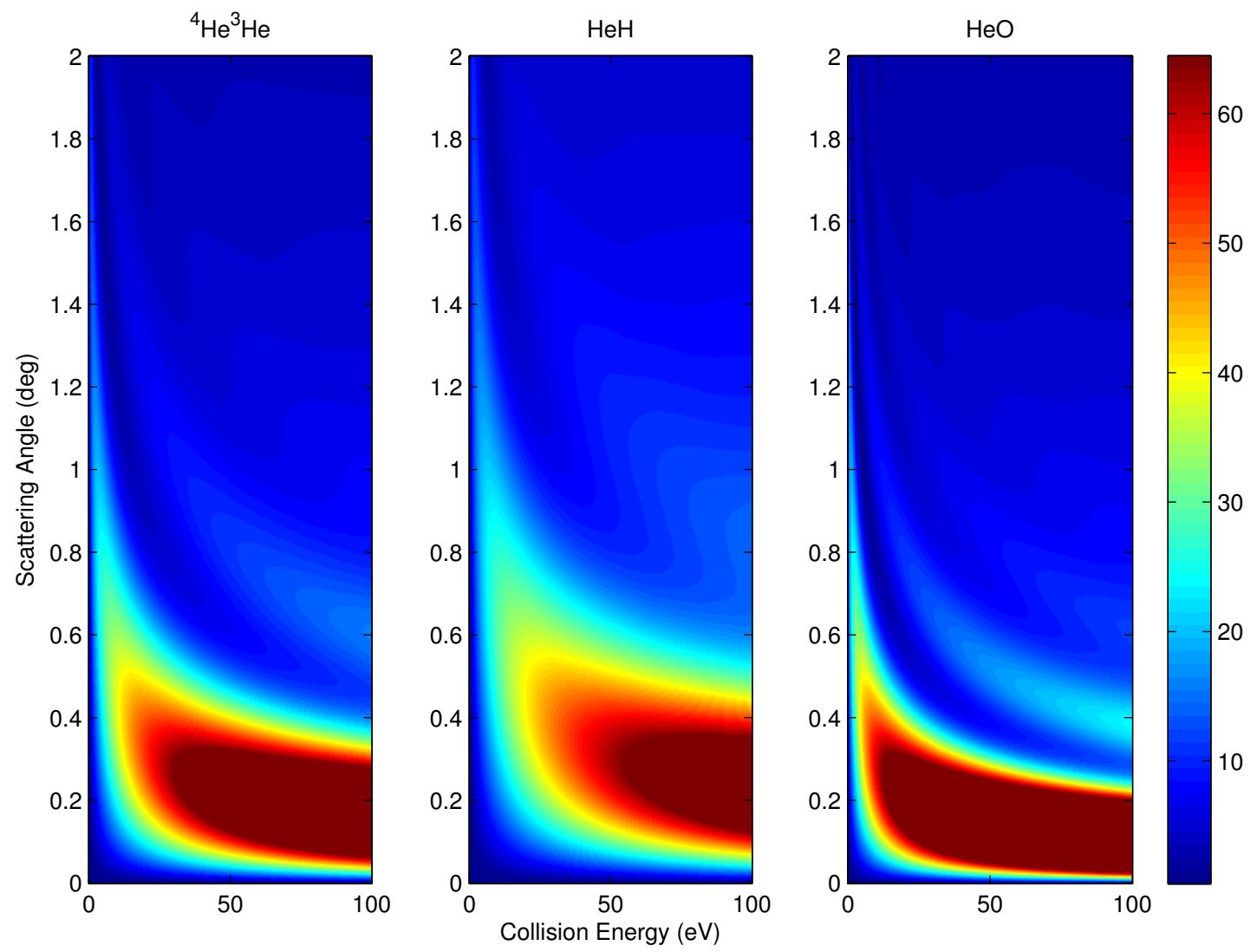

Fig. 2.10: Scattering angle probability densities for collisions of ${ }^{4} \mathrm{He}+{ }^{3} \mathrm{He}, \mathrm{He}+\mathrm{H}$, and $\mathrm{He}+\mathrm{O}$ up to a center of mass energy of $100 \mathrm{eV}$.

1.5 , and $5 \mathrm{keV}$. For illustration of the experimental limitations in obtaining integrated total cross sections, the three minimal detection angles reported $[29,32,33]$ are also shown, demonstrating the vast amount of total cross section probability located within extremely small scattering angles. In the extreme case of the $5 \mathrm{keV}$ collision detected with the minimal detection angle of $0.12^{\circ}$ the integrated total cross section would $25 \%$ of the actual value as $75 \%$ of the scattering angle probability occurs at angles smaller than $0.12^{\circ}[10]$.

The average scattering angle, $\bar{\theta}$ and $\bar{\theta}^{2}$, may also be found from the scattering 


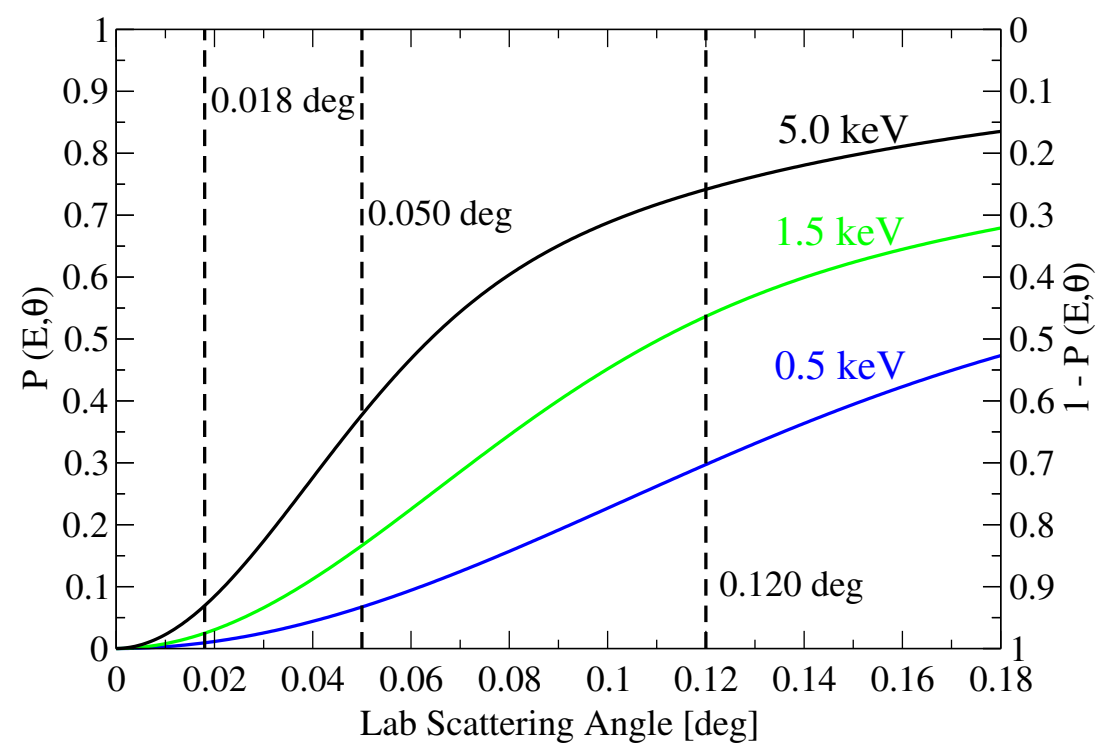

Fig. 2.11: Cumulative angular probability function $P(E, \theta)$ for $\mathrm{He}+\mathrm{H}$ collisions at 0.5 , 1.5, and $5 \mathrm{keV}$ laboratory frame energies. Experimental minimal angles $\theta_{m}$ reported from the literature are also shown with dashed lines.

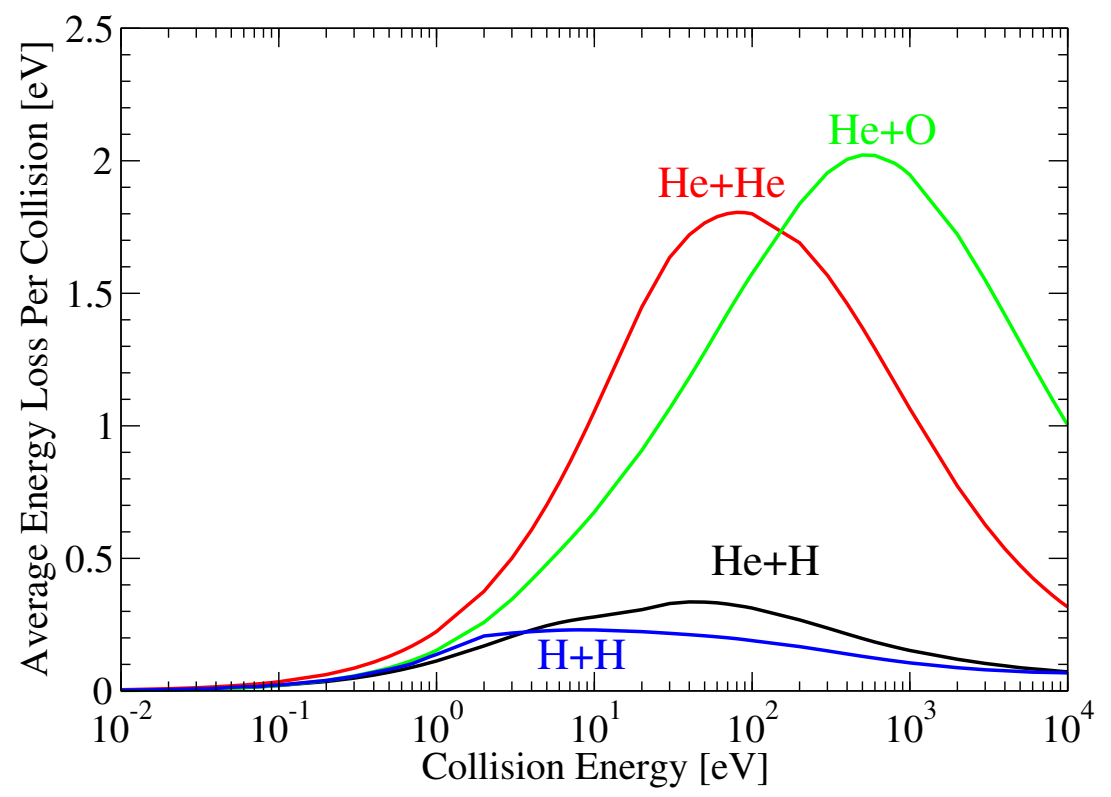

Fig. 2.12: Average energy loss per collision as a function of collision energy for $\mathrm{H}+\mathrm{H}$, $\mathrm{He}+\mathrm{H}, \mathrm{He}+\mathrm{He}$, and $\mathrm{He}+\mathrm{O}$ collisions. 
angle probability density as

$$
\bar{\theta}=\int_{0}^{\pi} \theta \rho(E, \theta) d \theta \quad \text { and } \quad \bar{\theta}^{2}=\int_{0}^{\pi} \theta^{2} \rho(E, \theta) d \theta .
$$

Utilizing the average scattering angle for a given collision, due to the domination of small angle scattering, the average energy loss per collision may also be found as

$$
\delta \bar{\epsilon}(\epsilon, \theta)=\epsilon \frac{2 m_{p} m_{t}}{\left(m_{p}+m_{t}\right)^{2}} \bar{\theta}^{2}
$$

where $m_{p}$ and $m_{t}$ are the projectile and target particle masses respectively. Eq. 2.46 is a very useful metric for determining average energy loss over large ensembles of particles. Fig. 2.12 displays the average energy loss per collision, Eq. 2.46, for collisions of $\mathrm{H}+\mathrm{H}$, $\mathrm{He}+\mathrm{H}, \mathrm{He}+\mathrm{He}$, and $\mathrm{He}+\mathrm{O}$. For all collision species there is a collision energy which is ideal for the transfer of energy as seen in the peak of Fig. 2.12. Additionally, collisions with heavier particles, in particular $\mathrm{He}+\mathrm{He}$ and $\mathrm{He}+\mathrm{O}$, have the largest transfer of energy due to their large masses.

With knowledge of the partial wave cross sections described above, several useful parameters involving energy-momentum transfer, heating rates, and atmosphere evolution may be obtained with more accurate results.

\subsubsection{Empirical Scaling Cross Sections}

In addition to the $a b$ initio partial wave cross sections described in Section 2.1.3, an empirical cross section database has also been developed to estimate differential and total cross sections for collisions between atoms and molecules which are not described well, either theoretically or experimentally, in the literature over the entire energy interval from $\mathrm{meV}$ to $\mathrm{keV}$. 
For atomic and molecular scattering theory in energy regimes where it is appropriate to utilize classical and semiclassical methods, it is common to display differential cross section data using reduced coordinates

$$
\tau=E \theta \quad \text { and } \quad \rho=\theta \sin \theta|f(E, \theta)|^{2},
$$

where $E, \theta$, and $|f(E, \theta)|^{2}$ are the center of mass energy, scattering angle and differential cross section respectively [34]. When a set of differential cross sections, covering a range of energies and scattering angles, is plotted $\rho$ vs $\tau$ for a given collision species the data tends to clump on a single line. Reduced coordinates provide a unique opportunity to extrapolate and incorporate missing data inside a given domain of $E$ and $\theta$ variables. In high-energy, forward scattering, the reduced coordinates arise from the leading terms of the impact parameter expansion and thus give a good approximation for the differential cross sections in this energy regime [34]. Fig. 2.13a displays differential cross sections, obtained using partial wave methods, for ${ }^{4} \mathrm{He}+{ }^{3} \mathrm{He}, \mathrm{He}+\mathrm{H}$, and $\mathrm{He}+\mathrm{O}$ over several collision energies and from scattering angles between $0^{\circ}$ to $10^{\circ}[10]$. As we described earlier, all the theoretical differential cross sections are in excellent agreement with experimental data. The trends of given collision species to clump together and lie along the same line is very clearly seen as three distinct lines in Fig. 2.13a. It was theorized that converting the reduced variables from being energy dependent to being velocity dependent through the inclusion of the reduced mass of the system, the individual collision line groupings seen in Fig. 2.13a may come together for all collisions species [10]. Transforming the variable $\tau$ from being energy dependent to being velocity dependent, $\tau \rightarrow \frac{\tau}{\mu}=\frac{E \theta}{\mu}$, and replotting the data from Fig. 2.13a, results in the grouping of the 

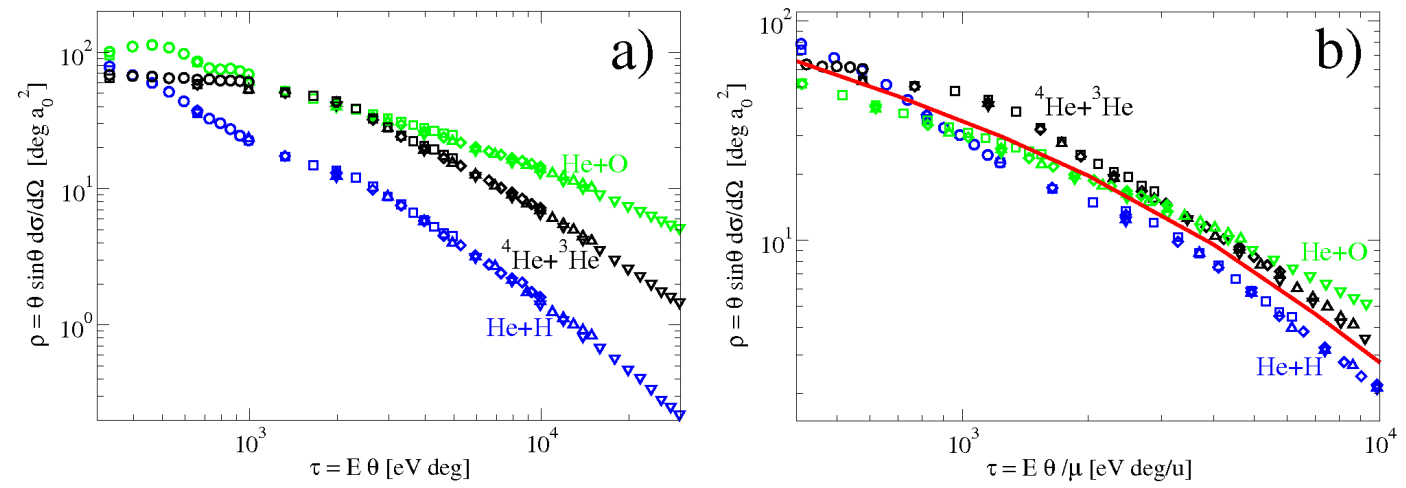

Fig. 2.13: Differential cross sections from $0^{\circ}$ to $10^{\circ}$ for $100 \mathrm{eV}$ (circles), $500 \mathrm{eV}$ (squares), $1 \mathrm{keV}$ (diamonds), $1.5 \mathrm{keV}$ (up triangles), and $3 \mathrm{keV}$ (down triangles) center of mass collision energies shown in (a) reduced energy coordinates and (b) reduced velocity coordinates for all non-identical collision species with a cubic fitting function shown as a red line. All differential cross sections utilized partial wave methods. 


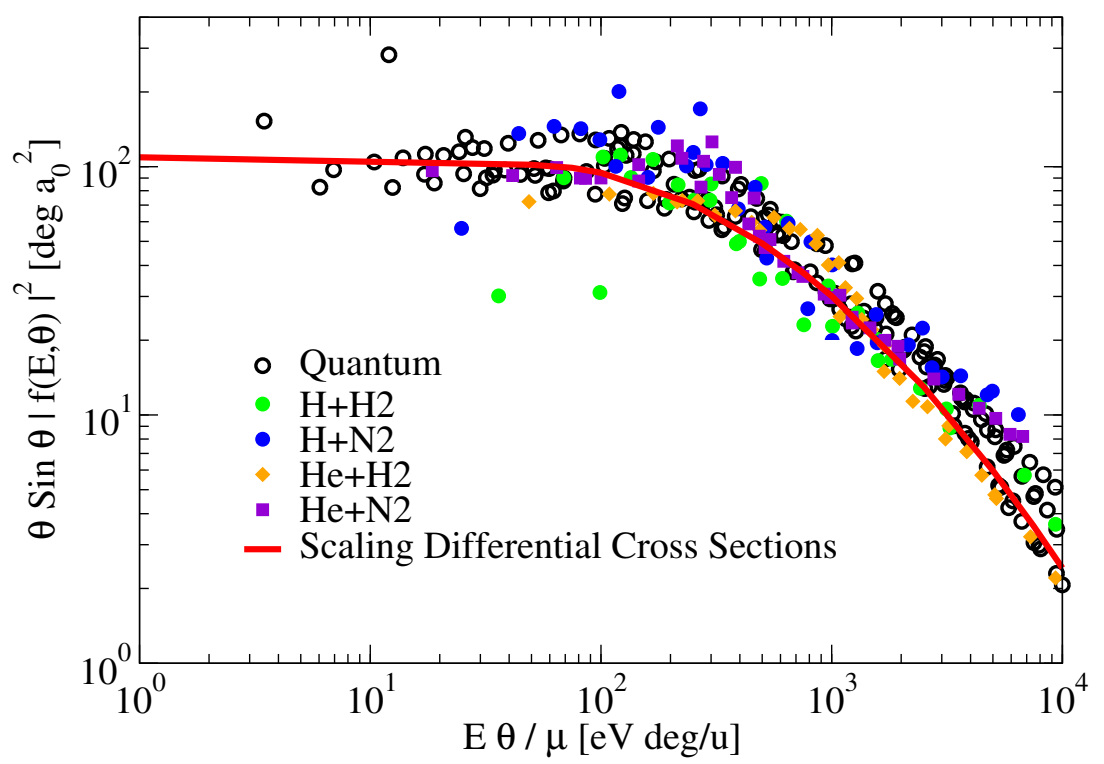

Fig. 2.14: Scaling differential cross section shown along with quantum mechanical partial wave differential cross sections for collisions of $\mathrm{H}+\mathrm{H}, \mathrm{He}+\mathrm{H}, \mathrm{He}+\mathrm{He}$, and $\mathrm{He}+\mathrm{O}$ shown as black circles, and experimental $\mathrm{H}+\mathrm{H}_{2}, \mathrm{H}+\mathrm{N}_{2}, \mathrm{He}+\mathrm{H}_{2}$, and $\mathrm{He}+\mathrm{N}_{2}$ differential cross sections. The scaling differential cross section fit is shown with a solid red line. All data are displayed in reduced velocity coordinates.

differential cross sections for all three quantum collisions as seen in Fig. 2.13b. A cubic function was fit to the data from all three quantum collisions in Fig. 2.13b for the energy-angular interval shown from 400 to $10^{4} \frac{\mathrm{eV} \mathrm{deg}}{\mathrm{u}}$.

To further investigate the extent and utility of these scaling differential cross sections, a basis of experimental atom-molecule differential cross section data was plotted together with the quantum partial wave differential cross sections to determine if the scaling procedure could be applied to more complicated atom-molecule collisions. Ex- 
perimental data for the collisions of $\mathrm{H}+\mathrm{H}_{2}, \mathrm{H}+\mathrm{N}_{2}, \mathrm{He}+\mathrm{H}_{2}$, and $\mathrm{He}+\mathrm{N}_{2}[35,29]$ were plotted in reduced velocity coordinates with the quantum partial wave differential cross sections and scaled by a uniform factor $\lambda$ resulting in a grouping of data from both theoretical atom-atom and experimental atom molecule collisions as seen in Fig. 2.14. The data in Fig. 2.14 were fit to a simple quadratic for large values of $\tau$, and a linear function for small values of $\tau$. Both fitting functions are applied to data in log-log scale. The scaling differential cross section may then be written as

$$
\begin{gathered}
|f(E, \theta)|^{2}=\frac{\lambda}{\theta \sin \theta} \exp \left[C_{1}\left(\log \frac{E \theta}{\mu}\right)^{2}+C_{2} \log \frac{E \theta}{\mu}+C_{3}\right], \quad \frac{E \theta}{\mu} \geq \tau_{0}, \\
|f(E, \theta)|^{2}=\frac{\lambda}{\theta \sin \theta} C_{4} \exp \left[C_{5}+C_{6} \log \frac{E \theta}{\mu}\right]+C_{7}, \quad \frac{E \theta}{\mu}<\tau_{0},
\end{gathered}
$$

where the fitting parameters $C_{1}, C_{2}, C_{3}, C_{4}, C_{5}, C_{6}$, and $C_{7}$ were found to be -0.13, 1.00, $2.70,10.0,2.04,-0.03$, and 32.3 respectively, and the cutoff parameter is $\tau_{0}=50.12$. The scaling constant is taken as $\lambda=1$ from atom-atom collisions and $\lambda=1.4$ for atom-molecule collisions. The units needed for Eqs. 2.48 and 2.49 are $\mathrm{E}$ [eV], $\theta$ [deg], and $\mu$ [amu], where the energy, scattering angle, and resulting differential cross section, $|f(E, \theta)|^{2}$ $\left[\mathrm{a}_{0}\right]$, are all in the center of mass frame.

To demonstrate the potential power of the scaling procedure, differential cross sections for collisions between oxygen atoms and the molecules $\mathrm{H}_{2} \mathrm{O}, \mathrm{CH}_{4}$, and $\mathrm{CO}_{2}$ have been calculated using this new scaling method and compared to experimental differential cross sections for laboratory frame energies of $100 \mathrm{eV}, 500 \mathrm{eV}$, and $1.5 \mathrm{keV}$ respectively [26]. Predicted and experimental cross sections are in an excellent agreement, taking into account that $\mathrm{O}+\mathrm{H}_{2} \mathrm{O}, \mathrm{O}+\mathrm{CH}_{4}$, and $\mathrm{O}+\mathrm{CO}_{2}$ differential cross sections are not included in the basic set of cross sections used to construct the scaling fit in Fig. 2.14. 


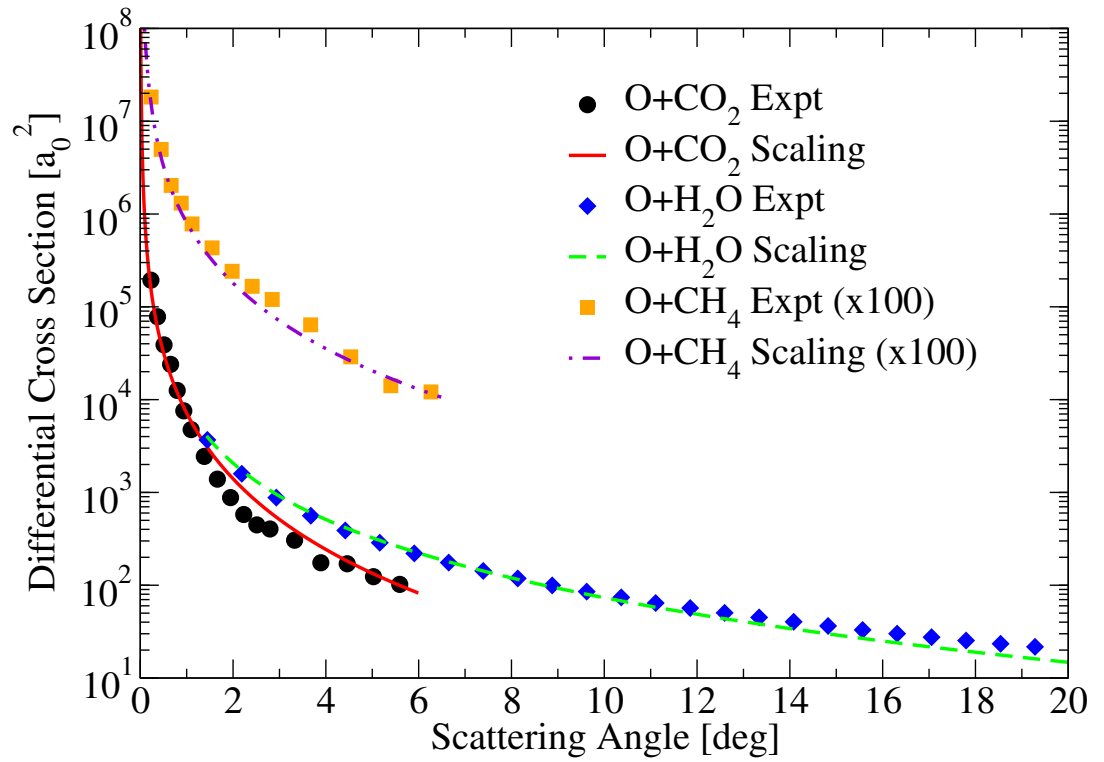

Fig. 2.15: Experimental differential cross sections for collisions of $100 \mathrm{eV} \mathrm{O}+\mathrm{H}_{2} \mathrm{O}, 500$ $\mathrm{eV} \mathrm{O}+\mathrm{CH}_{4}$, and $1.5 \mathrm{keV} \mathrm{O}+\mathrm{CO}_{2}$ shown as diamonds, squares, and circles. Scaling differential cross sections are shown as solid and dashed lines. All collision energies, scattering angles, and differential cross sections are shown in the laboratory frame. 
These specific collisions are important for several astrophysical environments as hot oxygen may be produced through dissociative recombination of $\mathrm{O}_{2}^{+}$[36] or as a minor species in the SW [37], through CX collisions with neutral gases. The target species are all commonly found in different astrophysical environments with $\mathrm{CO}_{2}$ being the major species in the lower Mars atmosphere [38], $\mathrm{H}_{2} \mathrm{O}$ making up $\sim 0.25 \%$ of the Earth's atmosphere [39] as well as being the largest constituent of cometary atmospheres [40], and $\mathrm{CH}_{4}$ being a major component in the atmosphere of Saturn's moon Titan [41]. Fig. 2.15 shows the experimental and scaling differential cross sections with excellent agreement for laboratory frame energies ranging from $100 \mathrm{eV}$ to $1.5 \mathrm{keV}$ and laboratory frame scattering angles up to $20^{\circ}$.

It is often the case that atom-molecule collisions may occur through inelastic channels, stimulating rotational and vibrational excitations in the molecular species and even their impact dissociation at high collision energies. In this work, inelastic atommolecule collisions all utilize the scaling differential cross section, which was constructed using elastic quantum mechanical cross sections as well as experimental atom-molecule cross sections, which naturally include both elastic and inelastic channels.

Total cross sections for unknown atom-atom and atom-molecule collisions were obtained through numeric integration of the scaling differential cross sections given by Eqs. 2.48 and 2.49. The numerical integration bounds used for all energies were set as $\theta_{\min }=0.01 \mathrm{deg}$ and $\theta_{\max }=170 \mathrm{deg}$ as both Eqs. 2.48 and 2.49 go to infinity as $\theta$ goes to $0 \mathrm{deg}$ and $180 \mathrm{deg}$. The choice of integration bounds was validated by comparing integrated scaling total cross sections with accurate computed quantum cross sections 
for collisions of $\mathrm{H}+\mathrm{H}, \mathrm{He}+\mathrm{H}, \mathrm{He}+\mathrm{He}$, and $\mathrm{He}+\mathrm{O}$. An average error of scaled cross section predictions is about $23 \%$ over the center of mass energies ranging from $1 \mathrm{eV}$ to $10 \mathrm{keV}$.

With the development of these new collisional cross section databases, accurate descriptions of energy-angular dependent collisions may be obtained using the quantum mechanical results for $\mathrm{H}+\mathrm{H}, \mathrm{He}+\mathrm{H}$, $\mathrm{He}+\mathrm{He}$, and $\mathrm{He}+\mathrm{O}$, while the scaled cross sections may be utilized for more complicated atom-molecule collisions. In the following sections these collisional cross section databases are used for accurate simulations in different astrophysical environments.

\subsubsection{Charge Exchange Cross Sections}

CX cross sections, in addition to elastic cross sections, are required for transport of ions and atoms in astrophysical environments. In particular, CX cross sections are used to determine neutralization properties of SW ions, allowing for accurate nascent distributions of ENAs. Plasma fusion research over the past several decades has resulted in vast amounts of data on CX collisions in the literature. The computation of CX cross sections has been well studied [42] but are out of the scope of this work. The CX cross sections used in this work were thus accumulated from the literature, utilizing both theoretical and experimental data to build a database for use in Monte Carlo simulations.

The main components of the SW are hydrogen ions, $\sim 96-98 \%$, and ions of helium, $\sim 2-4 \%$, with only trace amounts of heavier ions [43,37]. These SW ions are extremely

energetic with an average velocity of $437 \mathrm{~km} / \mathrm{s}$ [9] giving the ions an energy of $\sim 1$ 


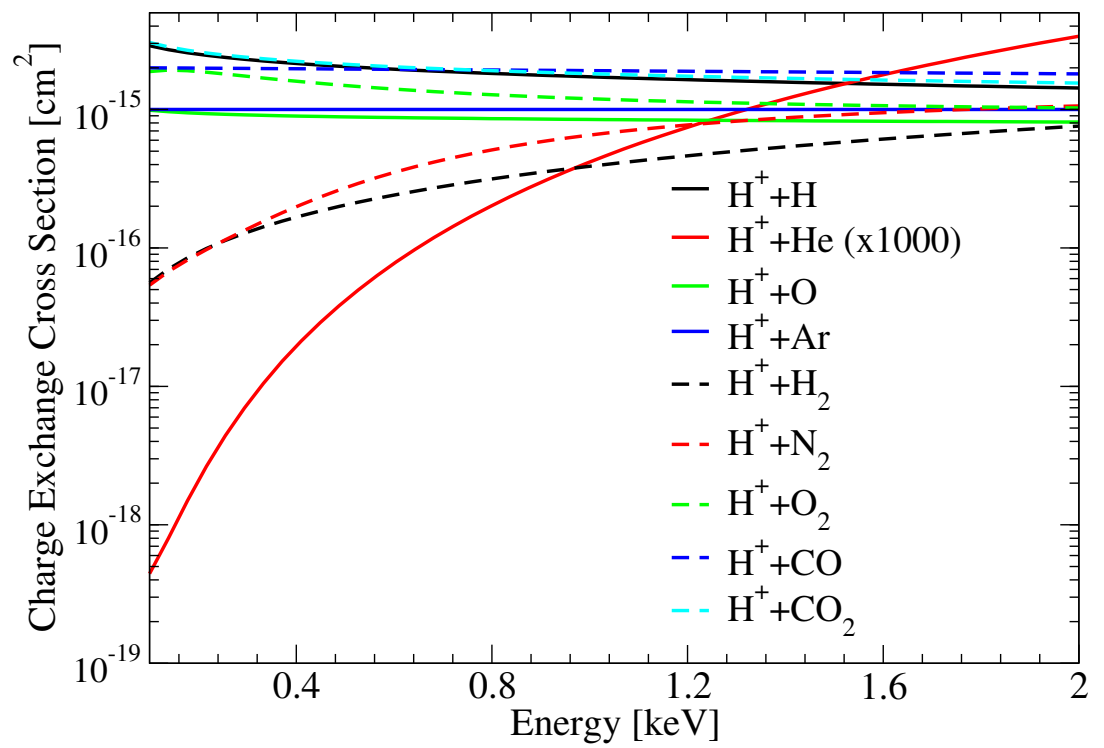

Fig. 2.16: Charge exchange cross sections for fast protons capturing an electron from atmospheric gases consisting of both atoms and molecules. A nascent energetic neutral hydrogen atom results from these collisions. The collision of $\mathrm{H}^{+}+\mathrm{He}$ is shown multiplied by a constant value of $10^{3}$ to show all data on a reasonable scale. 


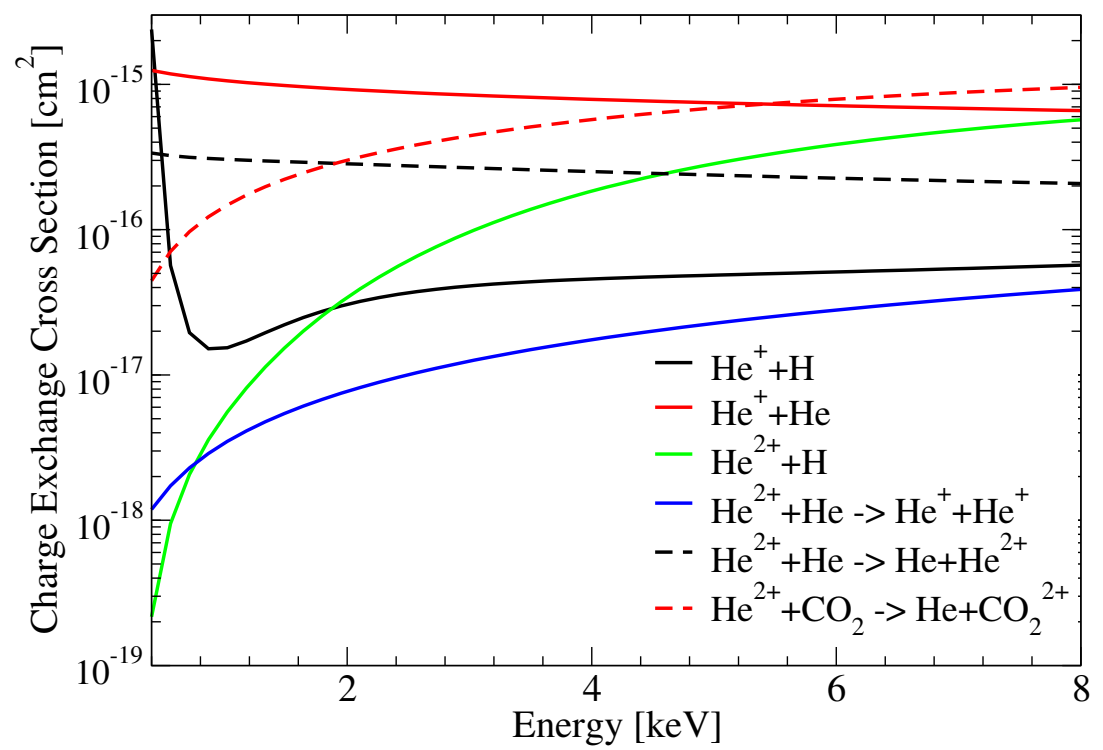

Fig. 2.17: Charge exchange cross sections for fast helium ions, consisting of both $\mathrm{He}^{2+}$ and $\mathrm{He}^{+}$, capturing one and two electrons from collisions with atmospheric gases consisting of both atoms and molecules. Collisions in which two electrons are transferred to $\mathrm{He}^{2+}$ during the collision are explicitly shown with the ion-atom system before and after the collision. 
$\mathrm{keV} / \mathrm{amu}$. It is then necessary to have knowledge of how these fast SW ions may CX with neutral gases creating nascent ENAs.

CX collisions between fast protons and neutral gases are extensively studied in the literature due to the ease of generating fast beams of protons in the lab for experiments and the vast abundance of ionized hydrogen in several astrophysical environments [44]. The two major astrophysical environments studied in this work include the atmosphere of Mars, Section 2.3, and the interstellar atmosphere, Section 2.4. The interstellar atmosphere is the simpler of the two, consisting predominately of neutral and ionized hydrogen $[45,46,3]$. In contrast, the atmosphere of Mars contains several neutral atomic and molecular species which may act as electron sources for neutralization of SW ions. The Mars atmosphere models utilized for this work consist of $\mathrm{H}, \mathrm{He}, \mathrm{O}, \mathrm{Ar}, \mathrm{H}_{2}, \mathrm{~N}_{2}$, $\mathrm{CO}$, and $\mathrm{CO}_{2}$ as these are the most prevalent atomic and molecular species [38]. Fig. 2.16 displays $\mathrm{CX}$ cross sections for hot protons colliding with neutral $\mathrm{H}, \mathrm{He}, \mathrm{O}, \mathrm{Ar}, \mathrm{H}_{2}$, $\mathrm{N}_{2}, \mathrm{O}_{2}, \mathrm{CO}$, and $\mathrm{CO}_{2}$ over the energy interval of $\mathrm{SW}$ protons [47-49]. The CX cross section for $\mathrm{H}^{+}+\mathrm{He}$ has been scaled by a factor of 1000 in Fig. 2.16 due to its extremely small value relative to the other CX cross sections.

Helium ions are also considered in this work and thus require knowledge of CX cross sections with neutral gases. The situation is slightly more complicated for helium ions as they may undergo one or two electron CX collisions depending on the target gases. With an initial beam of fast $\alpha$ particles, the process of neutralization may then be either a one step process

$$
H e^{2+}+X \rightarrow H e+X^{2+}
$$


or a two step process

$$
\begin{gathered}
H e^{2+}+X \rightarrow H e^{+}+X^{+} \\
H e^{+}+X \rightarrow H e+X^{+}
\end{gathered}
$$

with $X$ representing neutral atoms or molecules. Fig. 2.17 displays CX cross sections for hot helium ions colliding with neutral atoms and molecules, common in the astrophysical atmospheres of interest, for one and two electron transfer processes [48,50]. CX collisions between helium ions and neutral atoms or molecules not shown in Fig. 2.17 were approximated using similar cross sections in Fig. 2.17 as a complete database for helium ions does not exist in the literature for all neutral species of interest in this work.

While elastic neutral collisions utilized accurate energy-angular cross sections for Monte Carlo transport simulations in this work, CX collisions occur much less frequently and thus are treated in a simplified fashion. When a CX collision occurs the cross sections are extremely forward peaked [51-53] so that the nascent ENA maintains the same energy and velocity of its parent ion. The low frequency of CX collisions along with the extremely forward peaked differential CX cross sections leads to all CX collisions being treated as occurring with a scattering angle of $0^{\circ}$ and with no energy loss in this work. The CX cross sections described above are utilized in the Monte Carlo simulations described in Sections 2.3 and 2.4. 
Table 2.1: $\mathrm{H}+\mathrm{H}$ total cross sections analytic parameters

\begin{tabular}{ccc} 
Energy Interval $[\mathrm{eV}]$ & $\sigma_{0}\left[a_{0}^{2}\right]$ & $\alpha$ \\
\hline $0.01-0.07$ & 61.275 & 0.134 \\
$0.07-0.25$ & 85.378 & 0.102 \\
$0.25-5$ & 78.972 & 0.111 \\
$5-10$ & 68.640 & 0.135 \\
$10-50$ & 59.743 & 0.168 \\
$50-300$ & 55.041 & 0.194 \\
$300-420$ & 58.450 & 0.150 \\
$420-870$ & 51.792 & 0.289 \\
$870-1000$ & 50.963 & 0.407 \\
$1000-3000$ & 51.425 & 0.514 \\
$3000-5680$ & 67.559 & 0.751 \\
$5680-10000$ & 65.714 & 0.732 \\
\hline
\end{tabular}

Table 2.2: ${ }^{4} \mathrm{He}+\mathrm{H}$ total cross sections analytic parameters

\begin{tabular}{ccc}
\hline Energy Interval $[\mathrm{eV}]$ & $\sigma_{0}\left[a_{0}^{2}\right]$ & $\alpha$ \\
\hline $0.01-0.06$ & 61.896 & 0.087 \\
$0.06-0.36$ & 70.309 & 0.075 \\
$0.36-2.5$ & 55.364 & 0.105 \\
$2.5-19$ & 44.212 & 0.144 \\
$19-70$ & 38.394 & 0.179 \\
$70-470$ & 33.228 & 0.234 \\
$470-1000$ & 30.922 & 0.329 \\
$1000-3100$ & 30.978 & 0.447 \\
$3100-8400$ & 34.323 & 0.539 \\
$8400-10000$ & 38.446 & 0.592 \\
\hline
\end{tabular}


Table 2.3: ${ }^{4} \mathrm{He}+{ }^{4} \mathrm{He}$ total cross sections analytic parameters

\begin{tabular}{ccc} 
Energy Interval $[\mathrm{eV}]$ & $\sigma_{0}\left[a_{0}^{2}\right]$ & $\alpha$ \\
\hline $0.01-0.07$ & 136.095 & 0.057 \\
$0.07-0.50$ & 134.621 & 0.059 \\
$0.50-12$ & 104.992 & 0.093 \\
$12-46$ & 96.367 & 0.111 \\
$46-640$ & 85.722 & 0.148 \\
$640-1000$ & 84.640 & 0.177 \\
$1000-10000$ & 84.170 & 0.203 \\
\hline
\end{tabular}

Table 2.4: ${ }^{3} \mathrm{He}+{ }^{3} \mathrm{He}$ total cross sections analytic parameters

\begin{tabular}{ccc} 
Energy Interval $[\mathrm{eV}]$ & $\sigma_{0}\left[a_{0}^{2}\right]$ & $\alpha$ \\
\hline $0.01-0.07$ & 100.214 & 0.085 \\
$0.07-0.49$ & 134.304 & 0.056 \\
$0.49-12$ & 100.430 & 0.095 \\
$12-64$ & 89.177 & 0.121 \\
$64-640$ & 81.419 & 0.152 \\
$640-820$ & 80.388 & 0.181 \\
$820-10000$ & 79.863 & 0.210 \\
\hline
\end{tabular}


Table 2.5: ${ }^{4} \mathrm{He}+{ }^{3} \mathrm{He}$ total cross sections analytic parameters

\begin{tabular}{ccc} 
Energy Interval $[\mathrm{eV}]$ & $\sigma_{0}\left[a_{0}^{2}\right]$ & $\alpha$ \\
\hline $0.01-0.06$ & 60.900 & 0.067 \\
$0.06-0.35$ & 67.800 & 0.056 \\
$0.35-2.5$ & 57.971 & 0.075 \\
$2.5-15$ & 49.208 & 0.103 \\
$15-70$ & 45.143 & 0.123 \\
$70-500$ & 41.931 & 0.150 \\
$500-1000$ & 41.178 & 0.177 \\
$1000-10000$ & 41.175 & 0.210 \\
\hline
\end{tabular}

Table 2.6: ${ }^{4} \mathrm{He}+\mathrm{O}$ total cross sections analytic parameters

\begin{tabular}{ccc}
\hline Energy Interval $[\mathrm{eV}]$ & $\sigma_{0}\left[a_{0}^{2}\right]$ & $\alpha$ \\
\hline $0.01-0.04$ & 7.441 & 0.325 \\
$0.04-0.15$ & 33.009 & 0.178 \\
$0.15-1.5$ & 77.212 & 0.082 \\
$1.5-15$ & 78.369 & 0.080 \\
$15-40$ & 73.408 & 0.096 \\
$40-300$ & 68.502 & 0.117 \\
$300-1050$ & 67.371 & 0.131 \\
$1050-3000$ & 67.588 & 0.153 \\
$3000-10000$ & 70.206 & 0.188 \\
\hline
\end{tabular}




\subsection{Monte Carlo Transport}

With knowledge of accurate collisional cross sections, numerical simulations may be constructed which calculate several parameters describing how nascent energetic neutral atoms (ENAs) interact with astrophysical atmospheres. The process of energymomentum transfer and thermalization is inherently stochastic as a multitude of probabilistic events occur to bring the system from the initial, energetic, state to the final, thermal, state. To model such systems, Monte Carlo (MC) methods were employed using the newly calculated cross section databases described in Section 2.1 for atomic and molecular interactions, to accumulate macro statistics for the particular system of interest. Large ensembles of test particles are then used in the MC simulations to obtain probabilities for macro events such as atmospheric heating rates, secondary hot atom production, and in the case of planetary atmospheres, rates and fluxes of escape.

The general scheme of the MC simulations begins with the initialization of the test particles with initial energies $E_{0}$, positions $\vec{x}_{0}$, and directional cosines $\vec{u}_{0}$. The entire ensemble is then propagated a single step in the simulation. During this single simulation step, each test particle may either collide with an atmospheric particle, transferring energy to the target particle and changing the directional cosines, or be transported without a collision. The simulation continues, step-by-step, until every test particle in the ensemble has either thermalized with the atmospheric gases, or left the system. A simple flow diagram for the MC transport simulations is shown in Fig. 2.18.

For transport of ENAs in the interstellar medium, Section 2.4, the possibility for CX collisions to occur instead of elastic collisions is considered. In these simulations 


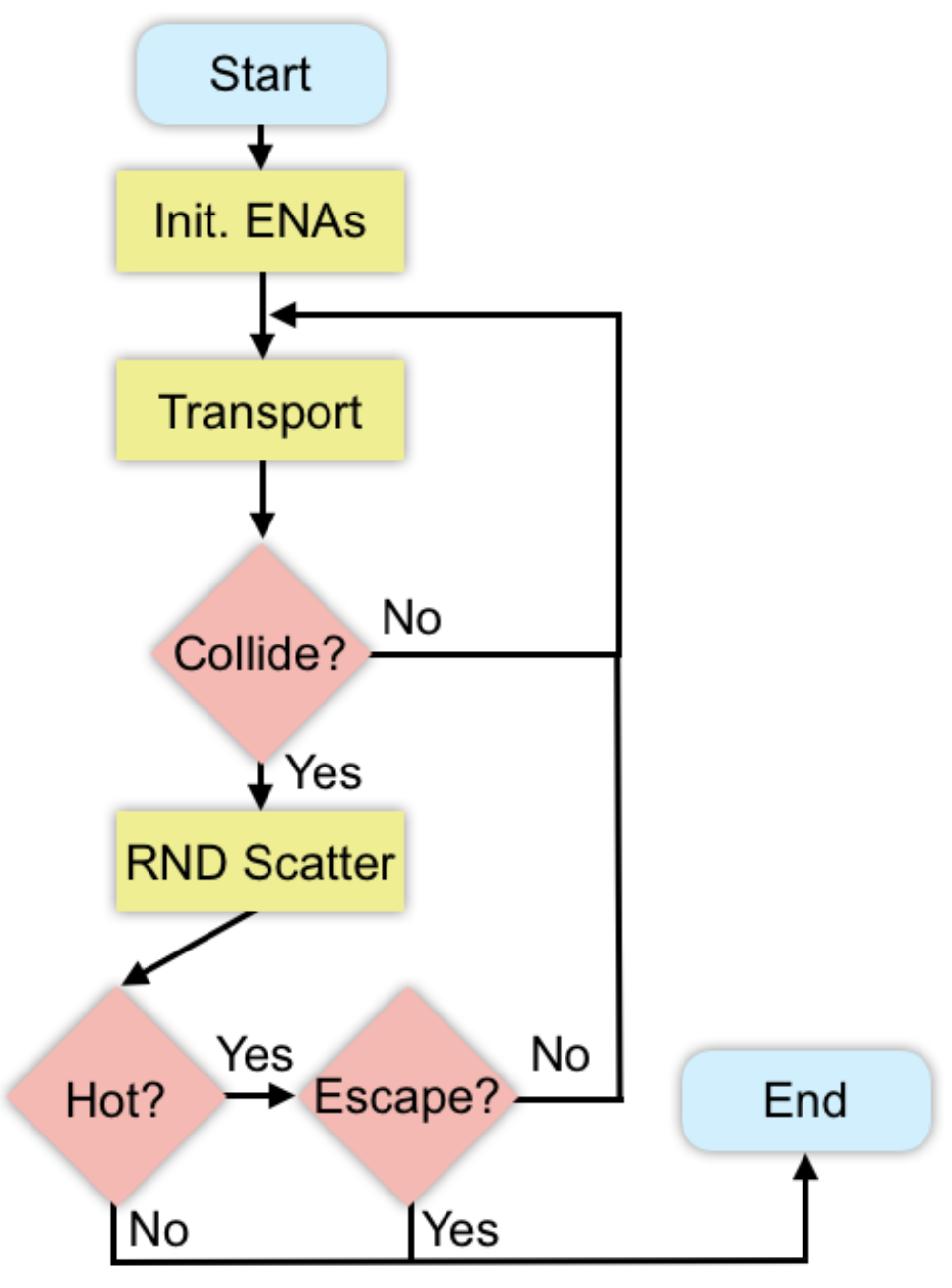

Fig. 2.18: Flow diagram for the Monte Carlo transport simulations in a planetary atmosphere. An ensemble of test particles is initiated with probabilistic initial energies, locations, and velocities. The ensemble is then transported a single step where each test particle may either collide, transferring some energy to the target particle during the collision, or not collide and be transported in a straight line. If a collision occurs during a transport step, the two exit criteria are checked; Is the test particle still more energetic than the local thermal energies? Is the test particle above the escape altitude? Once one of the escape criteria are met, the simulation ends for that test particle. 
special treatment is given to the transport of ions in the presence of a locally constant magnetic field.

For several aspects of the MC simulation, random numbers are required for determining stochastic parameters. Such stochastic parameters include random initial energies, initial positions, collision locations, scattering angles, and target species during collisions. A high quality, parallel random number generator was employed to ensure the random numbers used to determine stochastic events lack any pattern [54]. These random numbers are most easily used with cumulative probability distributions which are normalized to exist in the range $\in[0,1]$. For an arbitrary probability density distribution $y(x)$ where

$$
\int_{0}^{X} y(x) d x=1
$$

with $x \in[0, X]$ and $y(x) \geq 0 \forall x$, a cumulative probability is defined as

$$
Y(x)=\int_{0}^{x} y\left(x^{\prime}\right) d x^{\prime}
$$

where $Y(x)$ is a monotonically increasing function. To obtain a random parameter, the cumulative probability is numerically inverted so the input of a random number, $\eta$, uniformly distributed from $[0,1]$ results in the associated random parameter $x$ such that

$$
\eta=\int_{0}^{x} y\left(x^{\prime}\right) d x^{\prime}
$$

which when solved for $x$ yields $x(\eta)$. As an example, the cumulative probability for scattering angles shown in Fig. 2.11 may be used to obtain stochastic scattering angles for collisions between He+H. Fig. 2.19 displays the probability distributions for random scattering angles obtained during collisions of $\mathrm{He}+\mathrm{H}$ at laboratory energies of $500 \mathrm{eV}$, 


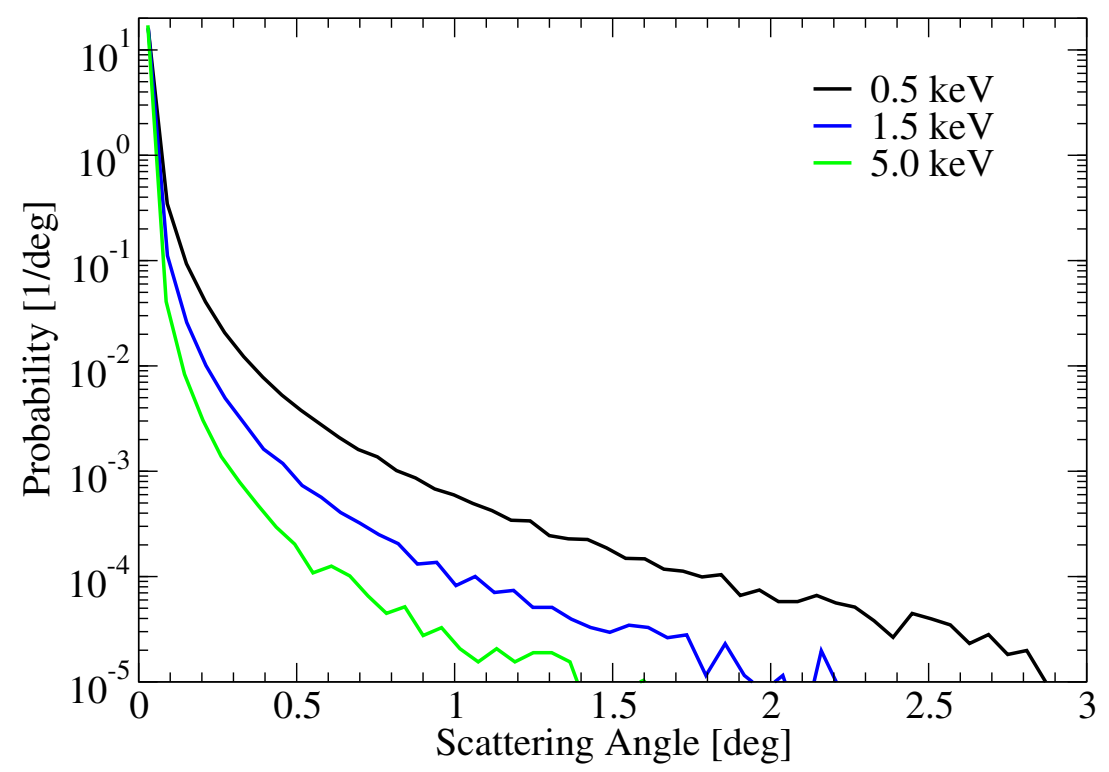

Fig. 2.19: Normalized probability distribution for random scattering angles for collisions of $\mathrm{He}+\mathrm{H}$ at $0.5,1.5$, and $5 \mathrm{keV}$ laboratory frame energies. The scattering angles shown are in the center of mass frame.

$1.5 \mathrm{keV}$, and $5 \mathrm{keV}$, acquired with samples of 10 million random angles per collision. Scattering angle probability in Fig. 2.19 can be seen moving from higher scattering angles to lower scattering angles as the collision energy increases. Although it is clear that small scattering angles dominate for even moderately energetic collisions, the low probability, high scattering angle tail to the distributions seen in Fig. 2.19 are extremely important for analysis of the reflected flux of projectile particles when considering $10^{4}$ to $10^{6}$ collisions per test particle depending on the astrophysical environment being studied. Stochastic parameters for other random variables during the simulations were acquired in a similar manner.

During MC transport simulations, the test particles are allowed to move in three- 
dimensions, resulting in realistic final distributions. After the initial parameters are set for the ensemble, all test particles are transported through the astrophysical atmosphere using a step-by-step method. At each simulation step, the total mean free path is determined for each test particle at its current location. Assuming there are a total of $N$ atmospheric species with with the test particles can collide, the mean free path can be written as

$$
\lambda(\vec{x}, E)=\frac{1}{\sum_{i}^{N} n_{i}(\vec{x}) \sigma_{i}(E)}
$$

where $n_{i}(z)$ is the density of the $i^{t h}$ atmospheric species at position $\vec{x}$ and $\sigma_{i}(E)$ is the collisional cross section between the ENA test particle and the $i^{\text {th }}$ atmospheric species. The step-size employed at a given position in the atmosphere is determined using this total mean free path. Following previously used MC methods [36], the step-size, $\Delta s$, utilized is either $20 \%$ of the total mean free path, $\lambda(\vec{x}, E)$ or some set small distance $s_{\text {min }}$, which ever value is smaller. The value $s_{\min }$ depends greatly on the environment being simulated and the resolution required for the data collected in that environment. For simulations in the atmosphere of Mars, Section $2.3, s_{\min }$ was set to $1 \mathrm{~km}$ as was previously done [36]. Simulations in the local interstellar medium atmosphere, Section 2.4, required $s_{\min }$ be set to $1 \mathrm{AU}$ which was found to be a good parameter to obtain high resolution results. This methodology ensures that the atmospheric densities, and thus the total mean free path, is near constant before and after the test particle is transported in the given environment.

With the total mean free path and step-size determined, the probability for a 
collision to occur within that simulation step, $\Delta s$, may be found as

$$
P_{\text {coll }}=1-\exp \left[\frac{-\Delta s}{\lambda(\vec{x}, E)}\right]
$$

which is a simplified expression of typical particle transport, determined in utilizing straight trajectory transport over these small step-sizes. The probability for no collision to occur, $P_{n c}$, can easily be found as

$$
P_{n c}=1-P_{\text {coll }}=\exp \left[\frac{-\Delta s}{\lambda(\vec{x}, E)}\right]
$$

It should be noted that during ion transport, Eq. 2.56 is no longer valid and further consideration is required as shown in Section 2.2.1. A uniform random number, $\eta$, is used to determine if a collision occurs in the interval $\Delta s$ such that

$$
\begin{aligned}
& \eta>P_{n c} \quad \text { Collision } \\
& \eta<P_{n c} \quad \text { No Collision. }
\end{aligned}
$$

If a collision does occur the exact location of the collision, $\Delta l$, may be found using Eq. 2.56 with $\eta=P_{\text {coll }}$ and solving for $\Delta s=\Delta l$, found to be

$$
\Delta l=-\lambda(\vec{x}, E) \log \eta
$$

where it is always the case that the distance to the collision location $\Delta l$ is less than the step-size $\Delta s$. For a given collision, the target species is determined using atmospheric mixing ratios such that

$$
\Gamma_{i}(z)=\frac{n_{i}(z) \sigma_{i}(E)}{\sum_{j}^{N} n_{j}(z) \sigma_{j}(E)}
$$

where $\sum_{i}^{N} \Gamma_{i}(z)=1$. An array was then formed using the calculated mixing ratios and a random number was selected to determine the target species. The scattering angle 
for a given collision was determined as discussed previously, with azimuthal scattering angles being randomly generated between 0 and $\pi$. With scattering angles $\theta$ and $\phi$, the directional cosine for the test particle velocity may be updated such that

$$
\begin{aligned}
& u_{x}^{\prime}=\frac{\sin \theta}{\alpha}\left[u_{x} u_{z} \cos \phi-u_{y} \sin \phi\right]+u_{x} \cos \theta, \\
& u_{y}^{\prime}=\frac{\sin \theta}{\alpha}\left[u_{y} u_{z} \cos \phi+u_{x} \sin \phi\right]+u_{y} \cos \theta, \\
& u_{z}^{\prime}=-\alpha \sin \theta \cos \phi+u_{z} \cos \theta,
\end{aligned}
$$

where $\alpha=\sqrt{1-u_{z}^{2}}$ and the normalized vectors $\vec{u}$ and $\vec{u}^{\prime}$ are the directional cosines before and after the collision [55]. In the instance of $u_{z}= \pm 1$, Eq. 2.61 reduces to

$$
\begin{aligned}
& u_{x}^{\prime}=\sin \theta \cos \phi, \\
& u_{y}^{\prime}= \pm \sin \theta \sin \phi, \\
& u_{z}^{\prime}= \pm \cos \theta,
\end{aligned}
$$

which is needed for the first collision in simulating planetary atmospheres if the projectiles are directly incident on the planet with a solar zenith angle of $0^{\circ}$. In the trivial case of no collision occurring for a given step, $\vec{u}^{\prime}=\vec{u}$.

The updated particle location, $\vec{x}^{\prime}$, is found as either the location of the previous collision, or in the case of no collision in the previous step, a distance $\Delta s$ from the previous step's position $\vec{x}$. Using the previous step's directional cosine, $\vec{u}$

$$
\vec{x}^{\prime}=\vec{x}+\Delta \vec{u}
$$

where $\Delta=\Delta l$ if a collision occurs and $\Delta=\Delta s$ if no collision occurs. It should be noted that Eq. 2.63 is only valid for neutral particle transport.

Following a collision, the energy lost by the test particle is calculated

$$
\delta \epsilon(\epsilon, \theta)=\epsilon \frac{2 m_{p} m_{t}}{\left(m_{p}+m_{t}\right)^{2}}(1-\cos \theta)
$$


using the laboratory frame energy of the test particle $\epsilon$, the center of mass frame scattering angle $\theta$, and the masses of the projectile and target particles, $m_{p}$ and $m_{t}$ [56]. In this work, the energy of the projectile particles is so much higher than that of the thermal atmospheric energy that we treat all target particles as being stationary in the atmosphere. Using the stationary atmosphere approximation along with conservation of energy, the energy loss determined from Eq. 2.64 is also the energy transferred to the target particle. If the energy transferred to the target particle is larger than the thermal energy of the atmosphere, the target species is considered a secondary hot atom or molecule. MC simulations investigating the interaction of fast particles with the atmosphere of Mars, Section 2.3, store information of nascent secondary hot atoms and molecules including their energy, altitude, and directional cosine if a given collision transferrs enough energy to the atmospheric atom or molecule to consider it hot.

The MC formalism described above is used extensively in this work for investigations into energy-momentum transfer, reflection, and secondary hot atomic and molecular fluxes in different astrophysical environments.

\subsubsection{Ion Transport}

The transport scheme for the astrophysical environments studied in this work follows SW ions as they become nascent ENAs through CX collisions with neutral gases. The ENAs are then transported as ballistic particles, undergoing elastic collisions until they either thermalize or leave the astrophysical environment. During energetic neutral transport in the interstellar atmosphere, Section 2.4, the thermal ion density is comparable to the thermal neutral density. These high ion densities result in comparable probabilities for 


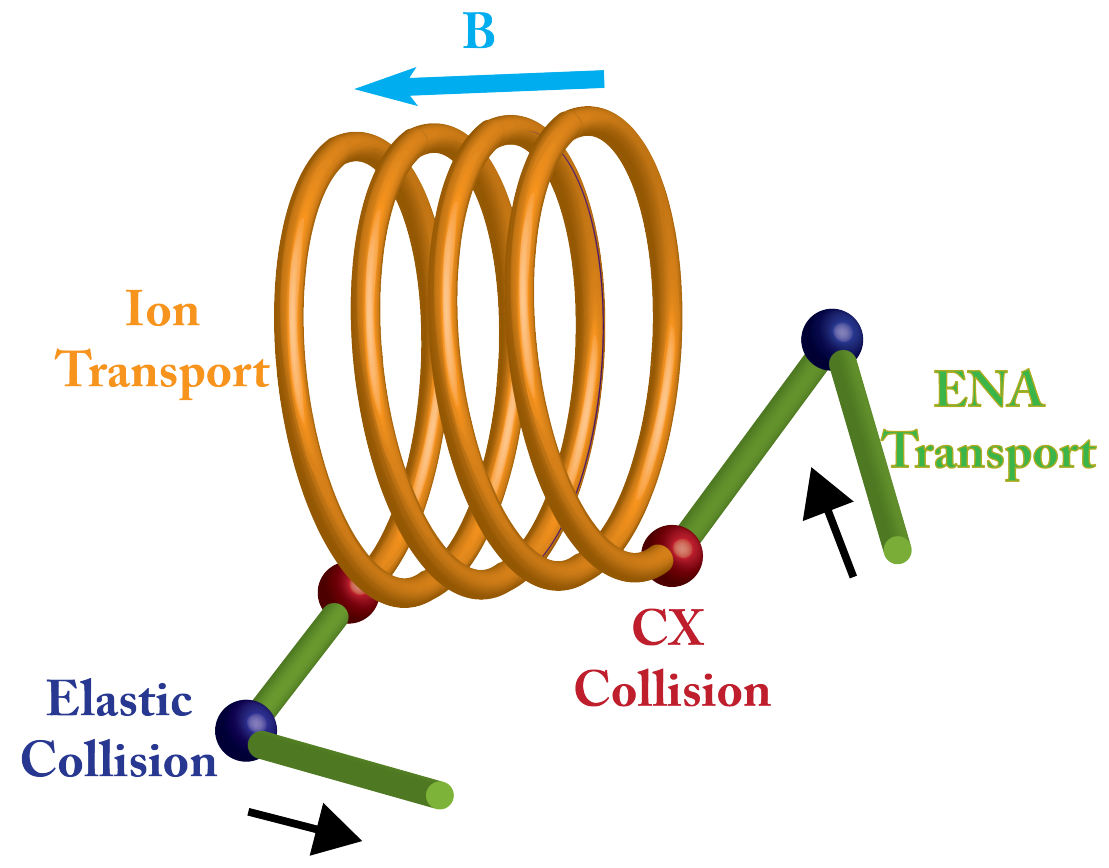

Fig. 2.20: Transport diagram for both neutral and ion transport. Energetic neutral particles may undergo charge exchange collisions with cold ions which results in helical transport about the local magnetic field $B$. Hot ions may again become energetic neutral atoms through undergoing charge exchange collisions with cold neutral gases. 
CX collisions to occur as compared with elastic collisions. Simulations in these high ion density environments then require considerations for nascent hot ion transport in addition to ballistic neutral atom transport.

A general transport diagram for neutral atom and ion transport is shown in Fig. 2.20. Energetic neutral atoms may collide either elastically or through CX collisions within the system. In the event of CX collisions the nascent ions are transported along helical trajectories according to the local magnetic field until another CX collision occurs, again creating a nascent ENA.

Transport of an ion in a constant magnetic field is most easily done by breaking the ion velocity into components which are parallel and perpendicular to the magnetic field. For an nascent ion with velocity $\vec{v}$ moving in the presence of a constant magnetic field $\vec{B}$, the parallel and perpendicular velocity components are expressed as

$$
\begin{aligned}
& \vec{v}_{\|}=\vec{B} \frac{\vec{v} \cdot \vec{B}}{|\vec{B}|^{2}} \\
& \vec{v}_{\perp}=\vec{v}-\vec{v}_{\|}
\end{aligned}
$$

with $\vec{v}_{\|}$and $\vec{v}_{\perp}$ being the parallel and perpendicular velocity components. The equation describing the motion of the ion can now be broken up into components which are parallel and perpendicular to the magnetic field. The parallel component simply moves in a straight trajectory as the Lorentz force for this component is zero

$$
\vec{r}_{\|}(t)=\vec{v}_{\|} t
$$

where $t$ is the time spent as an ion. To determine the equations of motion for the perpendicular component, an orthogonal basis is developed

$$
\hat{n}_{1}=\frac{\vec{v}_{\perp} \times \vec{B}}{\left|\vec{v}_{\perp} \times \vec{B}\right|} \quad \text { and } \quad \hat{n}_{2}=\frac{\hat{n}_{1} \times \vec{B}}{\left|\hat{n}_{1} \times \vec{B}\right|}
$$


such that both unit vectors $\hat{n}_{1}$ and $\hat{n}_{2}$ are themselves perpendicular to the magnetic field. The gyration radius, $R_{0}$, and frequency, $\omega_{0}$, are well known and defined as

$$
R_{0}=\frac{m\left|\vec{v}_{\perp}\right|}{|Z q||\vec{B}|} \quad \text { and } \quad \omega_{0}=\frac{Z q|\vec{B}|}{m}
$$

where $m$ is the ion mass, $Z$ is integer degree of ionization, and $q$ is the charge of an electron. Utilizing Eqs. 2.72 and 2.68, the perpendicular equation of motion can be written as

$$
\vec{r}_{\perp}(t)=R_{0}\left(\cos \left(\omega_{0} t\right)-1\right) \hat{n}_{1}+R_{0} \sin \left(\omega_{0} t\right) \hat{n}_{2}
$$

where the helical center has been shifted so that $\vec{r}_{\perp}(t=0)=0 \hat{n}_{1}+0 \hat{n}_{2}$. If the nascent hot ion was created at $\vec{r}_{0}$, then the entire equation of motion for the ion transport is written as

$$
\vec{r}(t)=\vec{r}_{0}+\vec{r}_{\|}(t)+\vec{r}_{\perp}(t) .
$$

Eq. 2.70 is used for transport of ions in the presence of a static magnetic fields as it provides a complete, time-dependent description.

Additional treatment for ion transport is required if the local environment is partially ionized, as is the case in the interstellar gas. In these partially ionized environments, a drag force is felt by the energetic ion due to the surrounding electron density. A simple diagram displaying this ion drag force is shown in Fig. 2.21. The ion drag force due to a hot ion being placed within a plasma was calculated as an exponential loss of energy with increasing time [57]

$$
E(t)=E_{0} \exp \left(\frac{-2 t}{\tau_{0}}\right)
$$




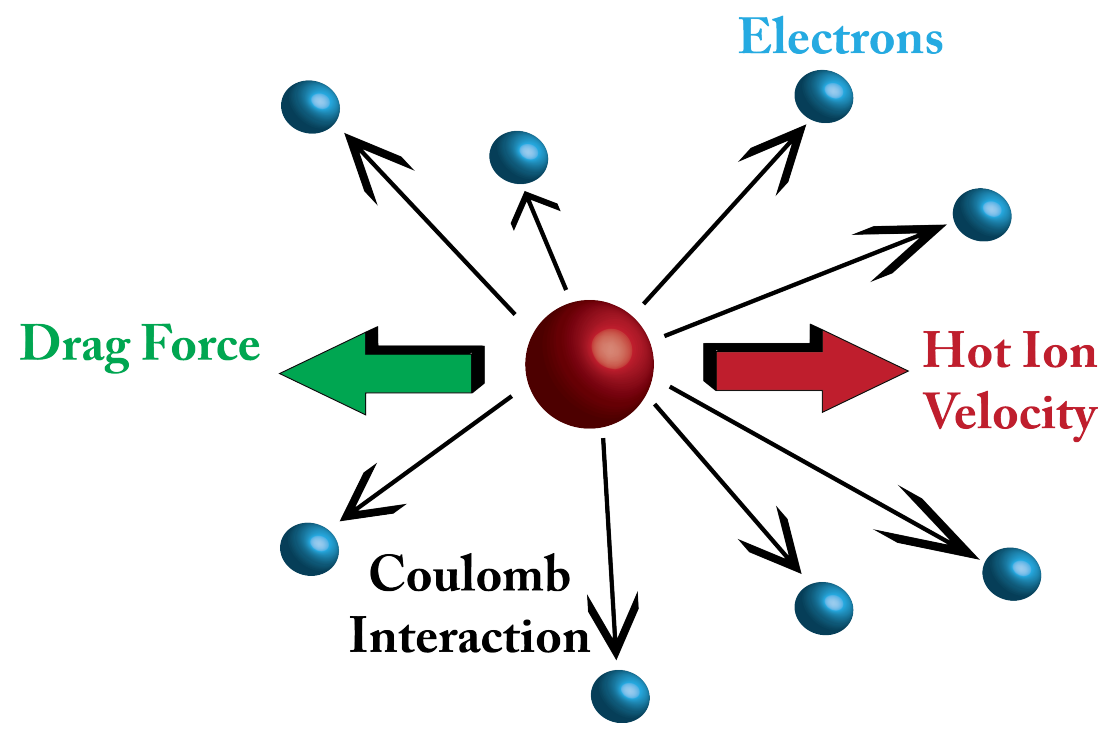

Fig. 2.21: Diagram illustrating the effective ion drag force felt by a hot ion being transported through a dilute, partially ionized gas.

where $E_{0}$ is the initial energy of the hot ion, $t$ is the time spent by the hot ion within the plasma, and the factor $\tau_{0}$ is defined as

$$
\tau_{0}=\frac{2 m_{i} m_{e} v_{i}^{3}}{16 \sqrt{\pi} k^{2} Z^{2} q^{4} n_{e} \log \frac{2}{\theta_{D}}} .
$$

Included within Eq. 2.72 are the masses of the hot ion and plasma electrons, $m_{i}$ and $m_{e}$, the ion velocity $v_{i}$, Coulomb's constant $k=\frac{1}{4 \pi \epsilon_{0}}$, the charge of the hot ion $Z$, the electron charge $q$, the thermal plasma electron density $n_{e}$, and the Debye length scattering angle $\theta_{D}$ [57]. The Debye length scattering angle is defined as

$$
\theta_{D}=\sqrt{\frac{\epsilon_{0} K_{b} T}{n_{e} q^{2}}}
$$

where $\epsilon_{0}$ is the permeability of free space, $K_{b}$ is the Boltzman constant, and $T$ is the thermal temperature of the plasma being traversed.

To illustrate the magnitude of the energy loss due to ion drag, energy loss per 


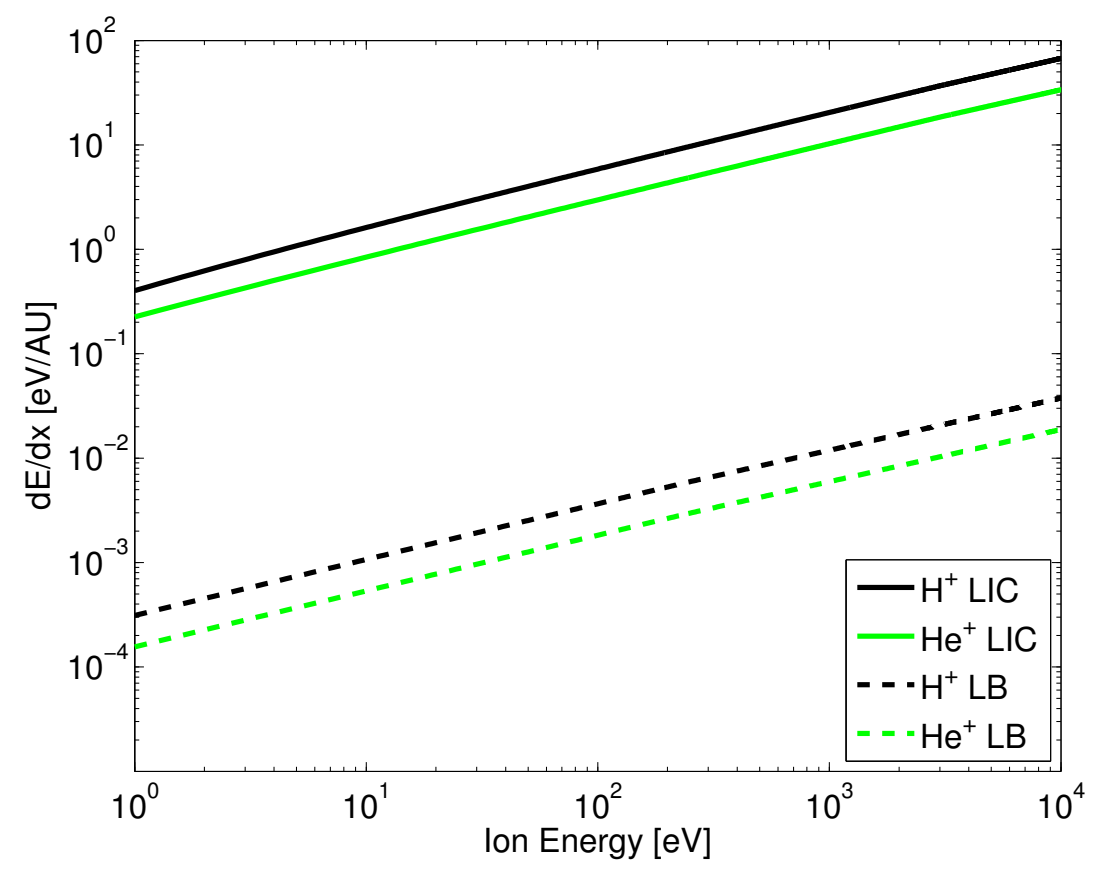

Fig. 2.22: Ion drag energy loss per transport distance for both hydrogen and helium ions traversing the model local interstellar cloud (LIC) and local bubble (LB).

transport distance as a function of ion energy is shown in Fig. 2.22 for hydrogen and singly charged helium ions in both the local interstellar cloud and the local bubble. Briefly, the local interstellar cloud consists of warm, $T=6000 \mathrm{~K}$, high neutral density, $n(H)=0.19 \mathrm{~cm}^{-3}$, with partial ionization, $n\left(H^{+}\right)=0.018 \mathrm{~cm}^{-3}$. In contrast, the local bubble contains hot, $T=10^{6} \mathrm{~K}$, completely ionized plasma with density $n\left(H^{+}\right)=0.005$ $\mathrm{cm}^{-3}[45,46,3]$. Extensive details of these two astrophysical environments are discussed in Section 2.4. Fig. 2.22 demonstrates how the energy loss per distance transported changes drastically for different astrophysical environments with varying plasma densities. 
When the hot ion is transported step-by-step through an environment with a static magnetic field and substantial plasma densities, the formalisms for both helical transport and ion-drag energy loss are combined. For long periods of transport in these conditions the gyromagnetic radius slowly decreases as the energy decreases due to the ion drag force. Small step sizes during transport and recalculation of ion transport properties for hot ions with constantly decreasing energies ensures proper numerical results during ion transport. These methods are utilized extensively for transport in the interstellar atmosphere Section 2.4.

\subsubsection{Frame Transformations}

Elastic collisions, in which energy is conserved between the projectile-target system and no change to internal energy states occurs, are the dominant collision types within the energy region of SW ions and the nascent ENAs they create [58]. An accurate treatment of collisional energy relaxation requires a transformation of the collision parameters from being in the center of mass frame to the frame related to the thermalized bath gas. The center of mass frame parameters are stochastic values because of the thermal motion of the bath gas. The averaging over thermal motion can be done using the Boltzmann kinetic equation $[59,60]$ or generating random thermal collision parameters in the $\mathrm{MC}$ simulation of the relaxation process [61]. A significant simplification can be applied to the frame transformation of fast particles when the motion of the bath gas can be neglected and the bath gas frame is considered as the laboratory frame.

It is common to express a two body system which interacts through a potential

$V(\vec{r})$ in the center of mass frame where the motion of the center of mass $\vec{R}$ of the 

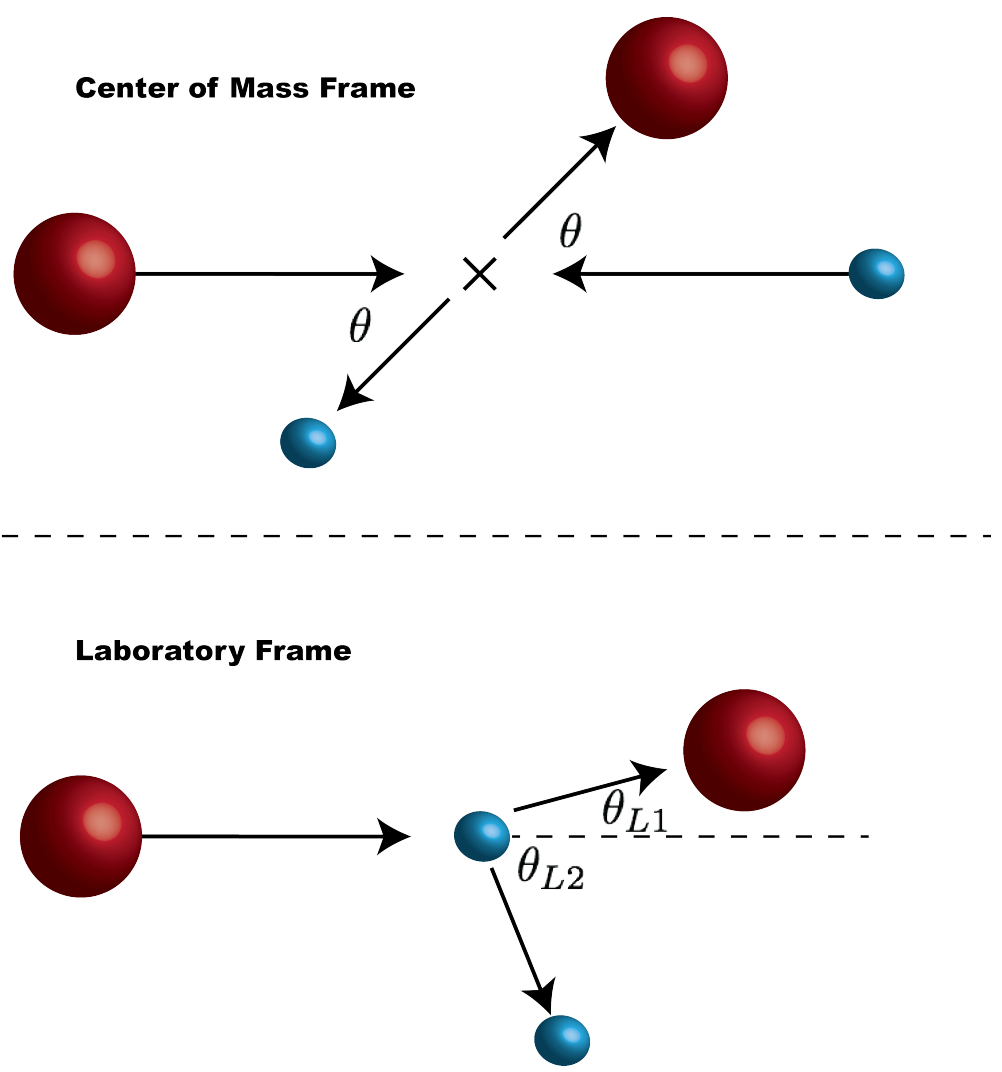

Fig. 2.23: Diagrams displaying the same collision in both the center of mass and laboratory frames. 
system is decoupled from the relative motion of the two bodies with the interparticle radius-vector $\vec{r}$. In general, given two particles interacting via a potential $V(\vec{r})$, the Hamiltonian for the system can be written as

$$
\hat{H}=\frac{p_{1}^{2}}{2 m_{1}}+\frac{p_{2}^{2}}{2 m_{2}}+V(\vec{r})
$$

for particles 1 and 2 with momenta $p_{1}$ and $p_{2}$ and masses $m_{1}$ and $m_{2}$. Eq. 2.74 describes the system in the inertial laboratory frame where the positions and motions of both particles are described relative to a stationary origin in the laboratory. The equivalent Hamiltonian can be written in the center of mass frame which describes the motion of the center of mass $\vec{R}$ with total mass $M=m_{1}+m_{2}$ and the relative motion of the two particles $\vec{r}$ with reduced mass $\mu=\frac{m_{1} m_{2}}{m_{1}+m_{2}}$ as

$$
\hat{H}=\frac{P^{2}}{2 M}+\frac{p^{2}}{2 \mu}+V(\vec{r})
$$

where $\vec{P}=M \dot{\vec{R}}$ and $\vec{p}=\mu \dot{\vec{r}}$. It is convenient to further define an inertial frame where $\dot{\vec{R}}=0$ and the resulting Hamiltonian of Eq. 2.75 becomes one dimensional

$$
\hat{H}=\frac{p^{2}}{2 \mu}+V(\vec{r})
$$

Conversions between the two reference frames are well known [56] and detailed below.

The total cross section for a given collision is the same in both the laboratory and center of mass frames since the actual scattering of a particle is not frame dependent. The differential cross section, on the other hand, differs when moving from the center of mass to the laboratory frame with a direct relationship between the two found as

$$
{\frac{d \sigma\left(\theta_{L}\right)}{d \Omega}}_{L a b}=\frac{d \sigma(\theta)}{d \Omega} C M_{C M} \frac{\left(1+2 \frac{m_{p}}{m_{t}} \cos \theta+\left(\frac{m_{p}}{m_{t}}\right)^{2}\right)^{3 / 2}}{1+\frac{m_{p}}{m_{t}} \cos \theta}
$$


where $m_{p}$ and $m_{t}$ are the projectile and target particle masses, $\theta_{L}$ is the scattering angle in the laboratory frame and $\theta$ is the scattering angle in the center of mass frame [56]. The laboratory frame scattering angles for the projectile, $\theta_{L 1}$, and the target, $\theta_{L 2}$, also have a direct relationship to the center of mass frame scattering angle as

$$
\theta_{L 1}=\tan ^{-1}\left(\frac{\sin \theta}{\cos \theta+\frac{m_{p}}{m_{t}}}\right) \text { and } \theta_{L 2}=\frac{1}{2}(\pi-\theta) .
$$

Conservation of energy also gives a relationship between collision energies in the center of mass and laboratory frames as

$$
E_{C M}=E_{L a b} \frac{\mu}{m_{p}}
$$

which results from taking into account the motion of the center of mass in the laboratory frame. Fig. 2.23 shows a diagrams for the same collision in both the center of mass and laboratory frames. The scattering angles in Fig. 2.23 were calculated using Eq. 2.78

with a mass ratio of $\frac{m_{p}}{m_{t}}=4$ and a center of mass scattering angle of $\theta=45^{\circ}$, resulting in laboratory scattering angles of $\theta_{L 1}=15^{\circ}$ and $\theta_{L 2}=68^{\circ}$.

Eqs. 2.77 through 2.79 are all used extensively in this work when simulating particle transport in different astrophysical environments where all collision processes occur in the center of mass frame and the resulting parameters are converted to the laboratory frame of the bath gases, to describe transport of the projectile particle in this bath gas.

\subsection{Precipitation of Energetic Neutral Atoms in the Atmosphere of Mars}

The evolution of planetary atmospheres is governed, in the simplest of terms, by energy input, transfer, and output. In planetary bodies without intrinsic magnetic fields, large 
amounts of energy may be supplied by solar or stellar wind ions into the atmosphere. Precipitating ions can capture electrons in collisions with atmospheric gases and become nascent energetic neutral atoms (ENAs) which penetrate deeply into the atmosphere before transferring their energy to the thermal gases present. It was estimated that precipitating ENAs deliver $10^{9} \mathrm{ev} \mathrm{cm}^{-2} \mathrm{~s}^{-1}$ to the atmosphere of Mars and is comparable to the energy input from extreme ultraviolet photons at solar minimum conditions [8]. Loss of neutral planetary atmospheres occur through both thermal (Jeans) escape and non-thermal energy transfer processes, leading to atomic and molecular escape. Significant numbers of atmospheric non-thermal processes are induced by precipitating solar and stellar wind ions. While thermal escape on Mars is efficient only for atomic and molecular hydrogen, the non-thermal energy transfer and escape may be the dominate source for evolution of heavier atmospheric constituents $[62,5]$.

The atmosphere of Mars has been the focus of investigations of planetary atmospheres for a long time, in particular, analysis of its current and past compositions helps shed light on the loss of liquid water which is thought to have once existed on the surface of the planet $[38,63-65]$. Previously calculated thermal and non-thermal escape rates of hydrogen, as well as sputtering and ion pickup, have led to estimates of an entire ocean of water with a global mean depth of $30 \mathrm{~m}$ being lost on Mars in the past 3.8 billion years [38]. Kinetics and energy relaxation involved in collisions between fast and thermal atoms are fundamentally important for the escape process and thus also atmospheric evolution $[66,21,60]$. Previous works have investigated the effects of SW protons precipitating into the atmosphere of Mars using both isotropic HS (HS) and 
angular dependent forward peaked cross sections [67], as well as with accurate quantum mechanical cross sections $[5,66,68,69]$, but accurate energy-angular dependent cross sections have never been fully used to study non-thermal, atom-atom and atom-molecule, energy transfer and induced escape fluxes in a planetary atmosphere. Precipitating ENAs are created through CX collisions between SW ions and atmospheric gases in the Martian atmosphere and in this work, we consider these ENAs as a source from non-thermal atomic and molecular escape and compare the ENA induced escape to previously reported escape fluxes.

The precipitation of ENAs into planetary atmospheres can be an efficient source of atmospheric heating as well as a production mechanism for secondary hot atoms and molecules. Secondary hot atoms and molecules created from ENAs essentially have nonthermal distributions and also contribute significantly to total planetary escape fluxes. Nascent ENAs created through CX collisions between SW ions and atmospheric gases maintain the vast majority of the SW ions velocity and thus have significantly large energies, ranging from hundreds of $\mathrm{eV} / \mathrm{amu}$ to several $\mathrm{keV} / \mathrm{amu}[9]$. As the nascent ENAs precipitate through the planetary atmosphere, their energy is transferred, via elastic and inelastic collisions, to the atmospheric gases with major atomic and molecular constituents being $\mathrm{H}, \mathrm{He}, \mathrm{O}, \mathrm{Ar}, \mathrm{H}_{2}, \mathrm{~N}_{2}, \mathrm{CO}$, and $\mathrm{CO}_{2}$ [38]. Extremely forward peaked differential cross sections for keV collisions lead to several thousand collisions and deep penetration into the planetary atmosphere before thermalizing [10]. Modeling of altitude profiles on energy deposition require realistic descriptions of energy transfer and thus accurate differential and total cross sections for binary collisions. 
Anisotropic quantum mechanical differential cross sections, unlike isotropic HS approximations, are extremely forward peaked for center of mass (CM) collision energies above $1 \mathrm{eV}$. We have calculated, with high accuracy, a majority of atom-atom collision cross sections. At the same time, ab initio calculations of atom-molecule cross sections at $\mathrm{keV}$ energies, such as atomic collisions with $\mathrm{CO}_{2}$ molecules, are not realistic and semi-empirical methods should be applied. Unknown cross sections of atom-molecule collisions between ENAs and some species of the Mars atmosphere were treated using the scaling cross sections from Section 2.1.4 to provide reasonable estimations of forward peaked differential cross sections as well as integrated total cross sections. These scaling cross sections are very useful in the atmosphere of Mars where $\mathrm{CO}, \mathrm{CO}_{2}, \mathrm{H}_{2}$, and $\mathrm{N}_{2}$ are large constituents and accurate quantum mechanical, ab initio computations at $\mathrm{keV} / \mathrm{amu}$ collision energies look as very formidable problems. All collisions between ENAs and these atmospheric molecules utilize the scaling cross sections, while all known atom-atom collisions $(\mathrm{H}+\mathrm{H}, \mathrm{He}+\mathrm{H}, \mathrm{He}+\mathrm{He}, \mathrm{He}+\mathrm{O})$ use computed ab initio quantum mechanical cross sections in this work.

Through use of quantum mechanical and scaling cross sections, accurate timedependent calculation of ENA transport, momentum transfer energy loss, secondary hot atomic and molecular production and escape was carried out using three dimensional Monte Carlo (MC) simulations, described in detail in Section 2.2, with large ensembles of test particles. Direct connections between the mechanisms of energy deposition and the intensities of induced escape fluxes for neutral atoms and molecules has been established using realistic cross sections, simulating quantum mechanical binary collisions, combined 
with classical MC transport Energy distributions for both thermalizing and escaping ENAs were found for ensembles of mono-energetic precipitating ENAs as well as realistic SW ion energy distributions [9]. Energy-deposition and escape flux comparisons between realistic anisotropic cross sections and isotropic HS models were made to further analyze differences in thermalization parameters between the two cross section models.

Accurate MC transport simulations depend on realistic neutral atmosphere profiles to determine altitude dependent heating rates, secondary hot atomic and molecular production, and reflection coefficients for ENAs. Three different realistic neutral atmosphere profiles were utilized in the MC transport simulations to observe how sensitive the resulting ensemble parameters were to neutral atmosphere densities. Atmosphere profiles, calculated from solving photochemical continuity equations with available in situ data, for minimum, maximum, and mean solar activity from 80-300 km were used exclusively in these simulations [38]. The atmosphere profiles were extrapolated up to an altitude of $800 \mathrm{~km}$ using an exponential fitting method which is commonly utilized in atmospheric modeling [70]. Altitude dependent, neutral atomic and molecular density profiles for minimum, mean, and maximum solar activity are shown with extrapolated high altitude fits in Figs. 2.24, 2.25, and 2.26. Drastic differences in the upper atmosphere profiles for minimum and maximum solar activity can be seen in Figs. 2.24 and 2.26 due to the complex photochemistry involved in several atomic and molecular processes and the large change in ionizing photon fluxes between the two solar activity extremes. The density profiles shown in Figs. 2.24, 2.25, and 2.26 are used and referenced extensively throughout this section. 


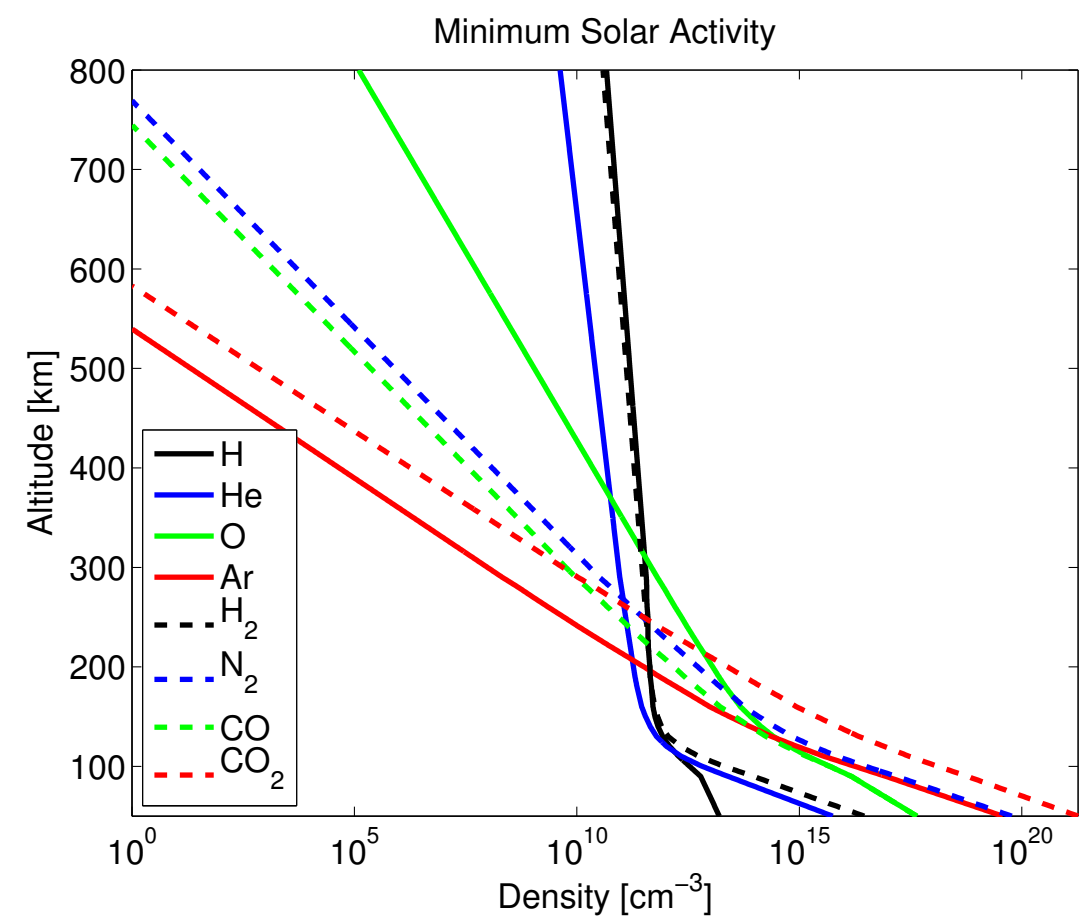

Fig. 2.24: Mars neutral density profiles for major atomic and molecular species at minimum solar activity. 


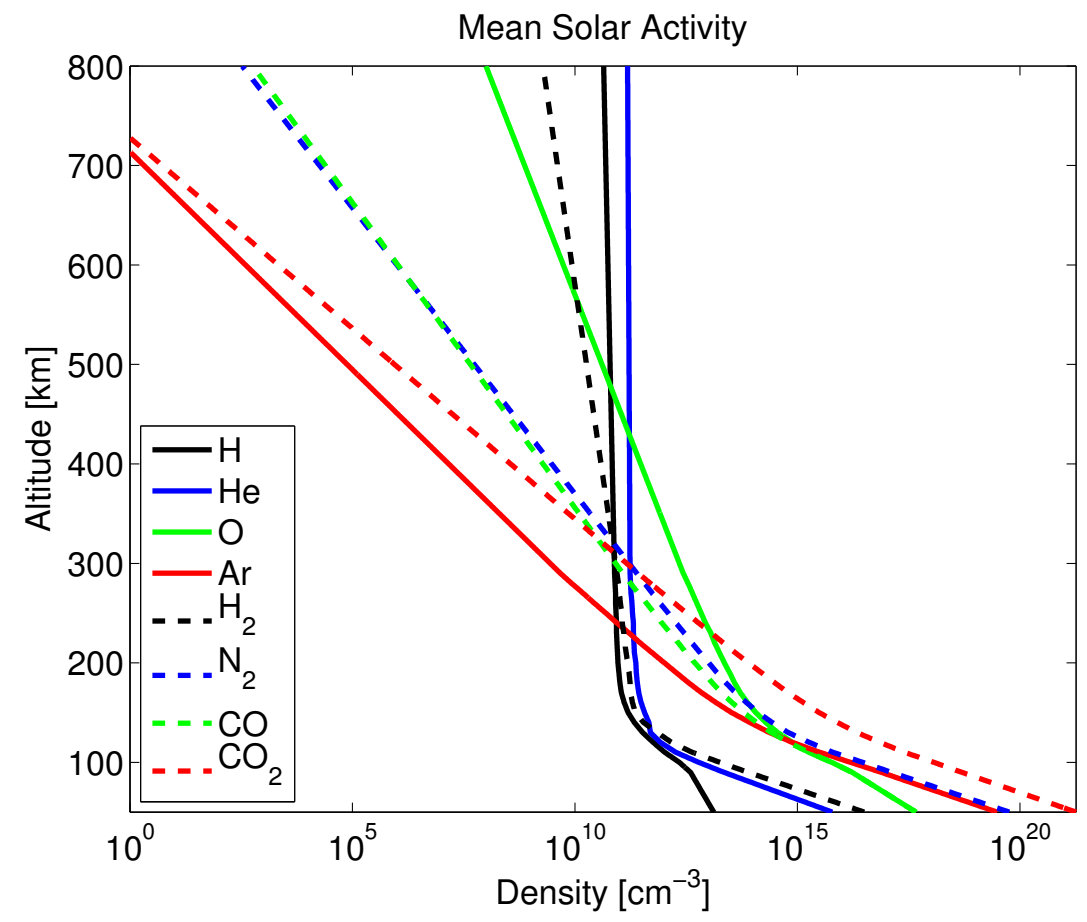

Fig. 2.25: Mars neutral density profiles for major atomic and molecular species at mean solar activity. 


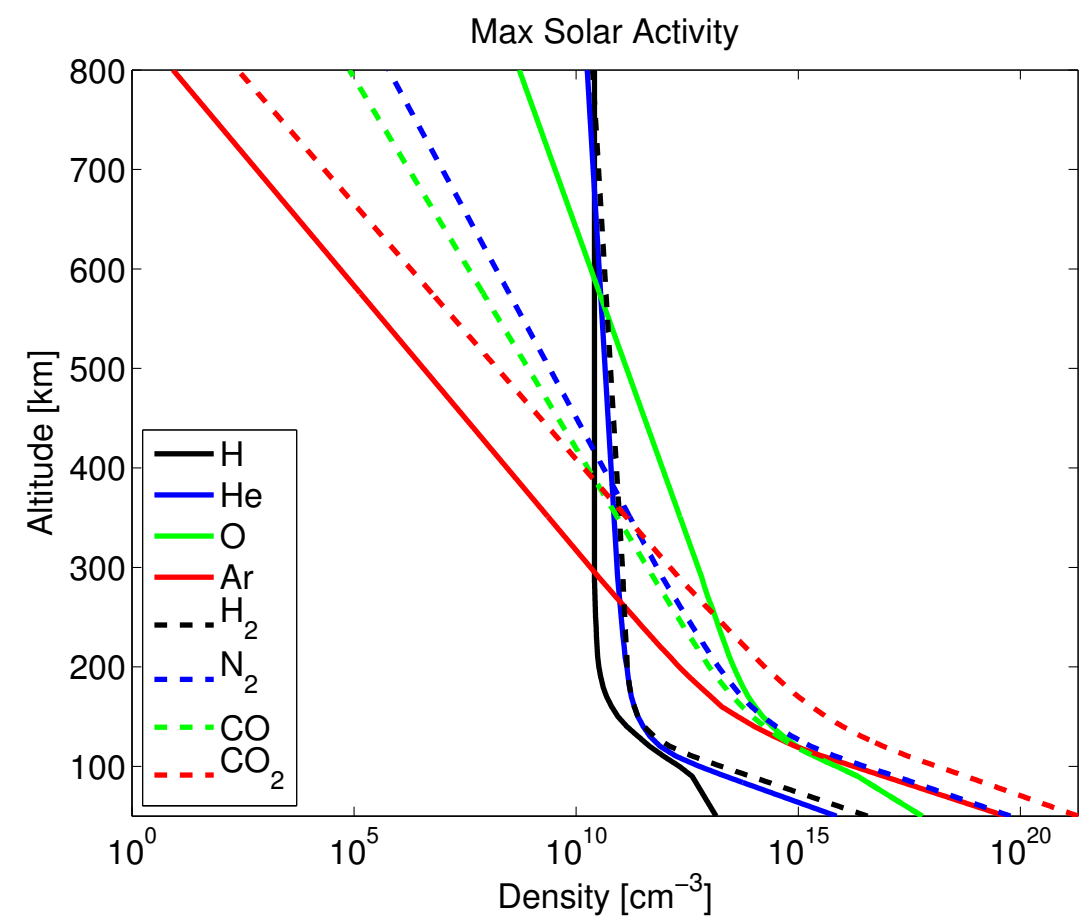

Fig. 2.26: Mars neutral density profiles for major atomic and molecular species at maximum solar activity. 
While differential cross sections are important for determining probabilistic scattering angles for a given collision, total cross sections are also required to determine if a collision occurs within a given transport step, Eqs. 2.55 and 2.56. Total cross sections were calculated using ab initio quantum mechanical methods for collisions of $\mathrm{H}+\mathrm{H}$, $\mathrm{He}+\mathrm{H}, \mathrm{He}+\mathrm{He}$, and $\mathrm{He}+\mathrm{O}[10]$ while all other collisions utilized numeric integration of the scaling amplitudes to obtain total cross sections. Fig. 2.27 shows the total cross sections used for MC simulations in the Martian atmosphere. Despite containing both quantum mechanical and scaling total cross sections, the curves in Fig. 2.27 are all relatively similar and are the same order of magnitude for all collision species over the energy interval from $1 \mathrm{eV}$ to $10 \mathrm{keV}$. Additionally, Fig. 2.28 displays energy dependent average scattering angles calculated for precipitating hydrogen and helium ENAs colliding with atmospheric atomic and molecular species. These forward peaked, heavily anisotropic cross sections result in both deep penetration of ENAs into the planetary atmosphere as well as several thousand collisions before thermalizing with the atmospheric gases. This effectively results in the energy deposition process involving significantly more layers of the atmospheric gas than with isotropic cross sections. The average scattering angles in Fig. 2.28 further demonstrate the forward peaked nature of these high energy collisions. Furthermore, the effectiveness of the scaling differential cross sections is also demonstrated in Fig. 2.28 as collisions which utilized this new method have average scattering angles which are similar to those obtained using quantum mechanical cross sections. It should also be noted that although these $\mathrm{keV}$ cross sections are extremely forward peaked, the low probable, large scattering angles are also important for transport and 

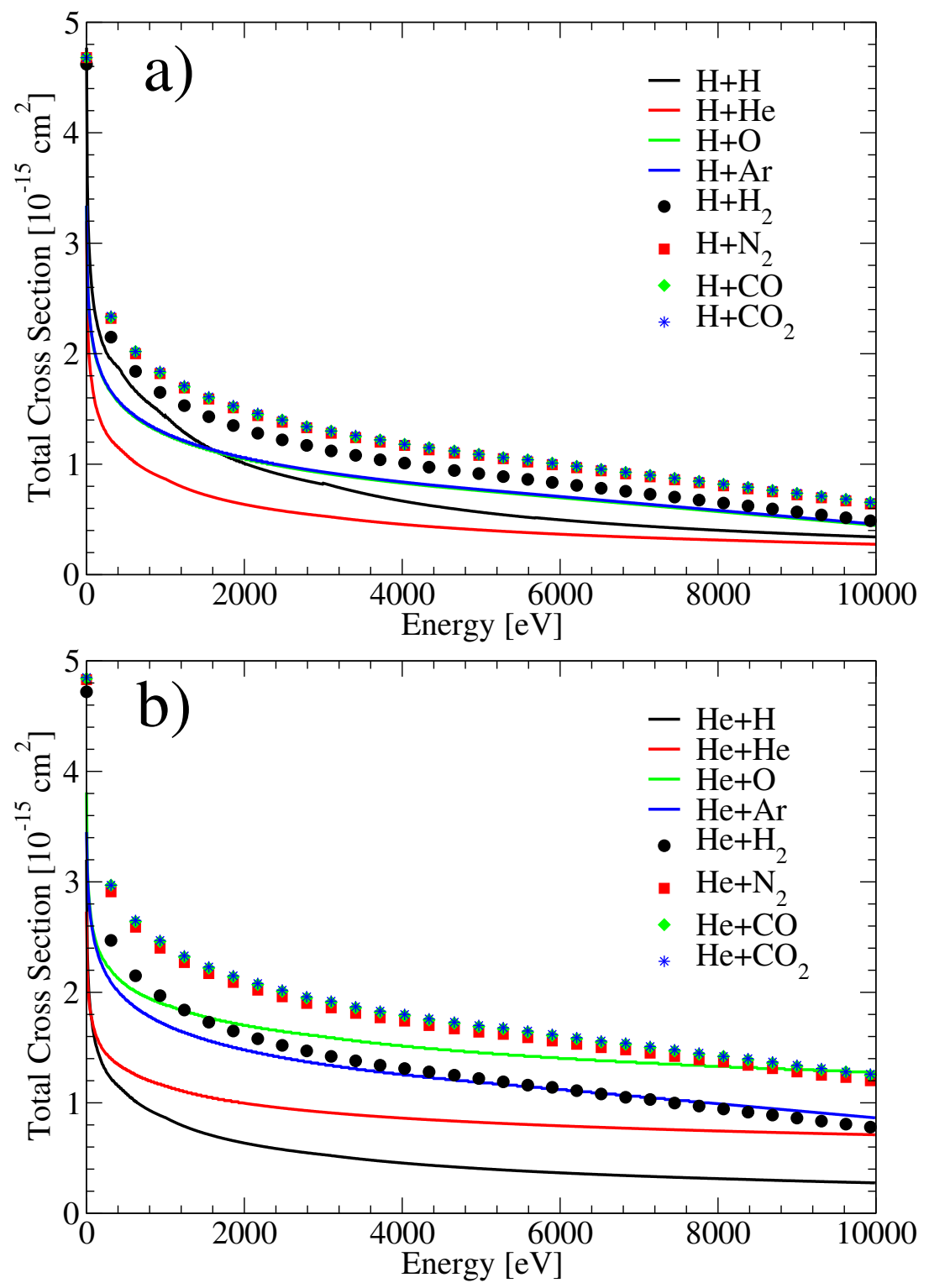

Fig. 2.27: Total cross sections obtained from numeric integration of the scaling differential cross sections as well as quantum mechanical partial wave analysis. a) displays total cross sections for hydrogen ENAs with the atmospheric constituents of Mars while b) displays the same data for helium ENAs. Collision energies are shown in the CM frame. 
thermalization as they are the main mechanism for production of fast secondary hot atoms and molecules within the atmosphere.

The main mechanism for the production of nascent ENAs in different astrophysical atmospheres is CX collisions between fast ions and thermal gases. The source of the energetic ions depends on the specific astrophysical environment. For example, SW ions may be a significant source of energetic ions for many planetary bodies while planets with intrinsic magnetic fields, such as Jupiter or Earth, also have magnetospheric ions which contribute significantly to ENA production. For the atmosphere of Mars, all ENAs are produced from SW ions as they CX with the neutral Mars atmosphere. Realistic models of the SW velocity distributions are then extremely important for simulating nascent ENA precipitation in the atmosphere of Mars. A statistical analysis of SW velocity data taken from 1989 through 2012 shows that the average SW energy ranges from $0.7 \mathrm{keV} / \mathrm{amu}$ to $1.5 \mathrm{keV} / \mathrm{amu}$ with the most energetic $\mathrm{SW}$ reaching energies of $4.2 \mathrm{keV} / \mathrm{amu}$ [9]. Theoretical mono-energetic ensembles of SW ions as well as the realistic average energy distributions of ions in the SW plasma were used to analyze how parameters of energy-deposition and precipitating fluxes change with initial energy distributions of SW ions and nascent ENAs.

In addition to SW velocity distributions and neutral atmospheric density profiles, accurate CX cross sections are required for determining altitude dependent production rates of nascent ENAs. The CX cross sections detailed in Section 2.1.5 were used for SW ions of hydrogen and helium interacting with the neutral gases of the Mars atmosphere. Charge exchange collisions occurring at $\mathrm{keV}$ energies, such as that of the $\mathrm{SW}$ ions, 

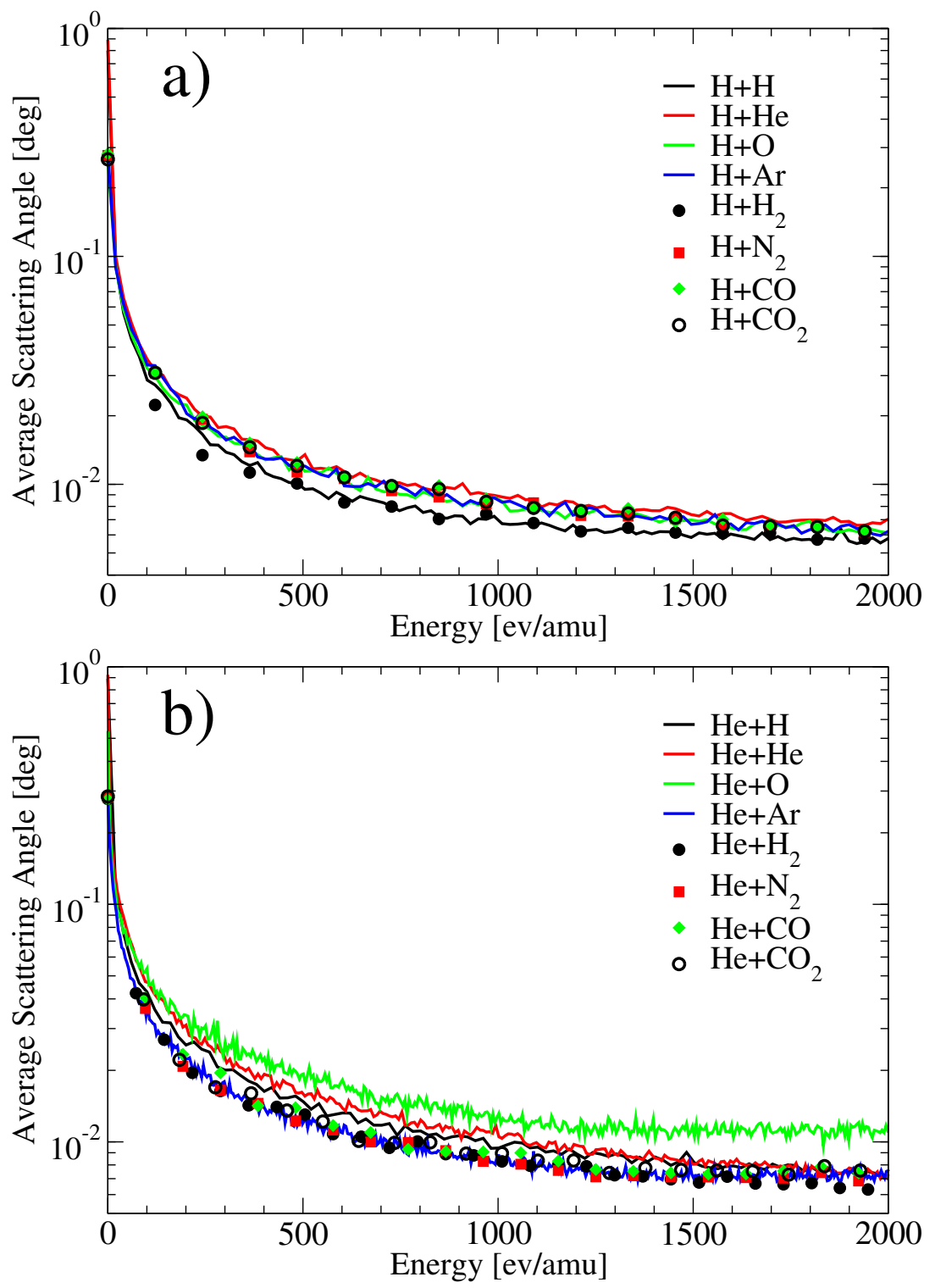

Fig. 2.28: Average CM scattering angles as a function of CM collision energy/amu for a) H ENAs and b) He ENAs with atmospheric constituents of Mars. 
are extremely forward peaked so that nascent ENAs maintain nearly the same velocity magnitude and direction of it's parent ion [51-53]. The probability of charge stripping processes is very low at $\mathrm{keV} / \mathrm{amu}$ collision energies and nascent ENAs, induced in CX collisions, propagate through the atmosphere as high speed neutral particles.

Although the SW ions are quickly converted to nascent ENAs, for consistency of the theoretical description, we also consider the energy loss of precipitating SW ions. For typical SW ion velocities, the energy losses related to the ionization and excitation of atmospheric atoms and molecules are very small and major energy losses occur in elastic collisions of precipitating ions with the ambient gas. The effect of SW ion elastic collisions was investigated to determine average energy losses prior to creation of ENAs from CX collisions. Energy-dependent elastic cross sections for $\mathrm{H}^{+}+\mathrm{H}$ were used for all atmospheric species in this investigation as actual elastic atom-ion cross sections were not readily available in the literature for all neutral species of interest. Energy loss in these ion collisions was determined using angular-energy dependent scaling differential cross sections to determine random scattering angles and thus random energy losses. It was found that incident SW ions undergo an average of 3 elastic ion-atom collisions and lose an average of $0.1 \mathrm{eV}$ from their initial energy before becoming nascent ENAs through CX collisions with atmospheric neutrals. These results were obtained using 100,000 particle ensembles which were initialized at $800 \mathrm{~km}$ using the model SW energy distribution [9] and were directly incident on the planet surface with a solar zenith angle of $0^{\circ}$. These energy losses were neglected since the typical energies of the precipitating $\mathrm{SW}$ ions is around a few $\mathrm{keV} / \mathrm{amu}$ making energy losses of $\sim 0.1 \mathrm{eV}$ insignificant. 
Nascent energetic neutral production fluxes, $f(z)$, were calculated using a simplified 1D atmospheric transparency integral

$$
f(z)=N_{s w} U_{s w} \frac{R_{0}^{2}}{R_{\text {mars }}^{2}} \exp \left(-\sum_{i} \int_{z}^{\infty} \sigma_{i}^{c x} n_{i}\left(z^{\prime}\right) d z^{\prime}\right) \sum_{i} \sigma_{i}^{c x} n_{i}(z)
$$

where $z$ is the altitude above Mars, $N_{s w}$ is the density of SW ions as observed in situ, scaled for $1 \mathrm{AU}$, with an average value of $5 \mathrm{~cm}^{-3}[71], U_{s w}$ is the velocity of the $\mathrm{SW}$ ions, $R_{0}$ is $1 \mathrm{AU}, R_{\text {mars }}$ is $1.5 \mathrm{AU}, \sigma_{i}^{c x}$ is the CX cross section for the $\mathrm{i}^{\text {th }}$ neutral species, and $n_{i}$ is the neutral density of the $\mathrm{i}^{\text {th }}$ neutral species at altitude $z$. To simplify calculations, a single, average SW velocity was used to determine ENA production altitudes for nascent hydrogen and helium. The average SW velocity used was $437 \mathrm{~km} / \mathrm{s}$ which corresponds to an energy of $1 \mathrm{keV} / \mathrm{amu}$. Cumulative distribution functions for ENA production altitudes, for all three atmosphere models, are shown in Fig. 2.29 for nascent hydrogen and helium ENAs. These cumulative distribution functions give a probabilistic measure for how deep SW ions penetrate into the atmosphere before becoming nascent ENAs. Fig. 2.29 shows how very few nascent ENAs are created above $300 \mathrm{~km}$ and below $150 \mathrm{~km}$ there are no longer any SW ions present as they have all become nascent ENAs. The minimum solar activity atmosphere model has the lowest number density for atmospheric species at a given altitude and thus has ENA production occurring lower in the atmosphere than the mean or maximum solar activity atmosphere models. Also, for all three atmosphere models, nascent helium ENA production occurred deeper in the atmosphere than nascent hydrogen ENA production which may be attributed to smaller average CX cross sections for helium ions. With altitude dependent production rates known, simulations of energy transfer in the atmosphere of Mars may be performed 


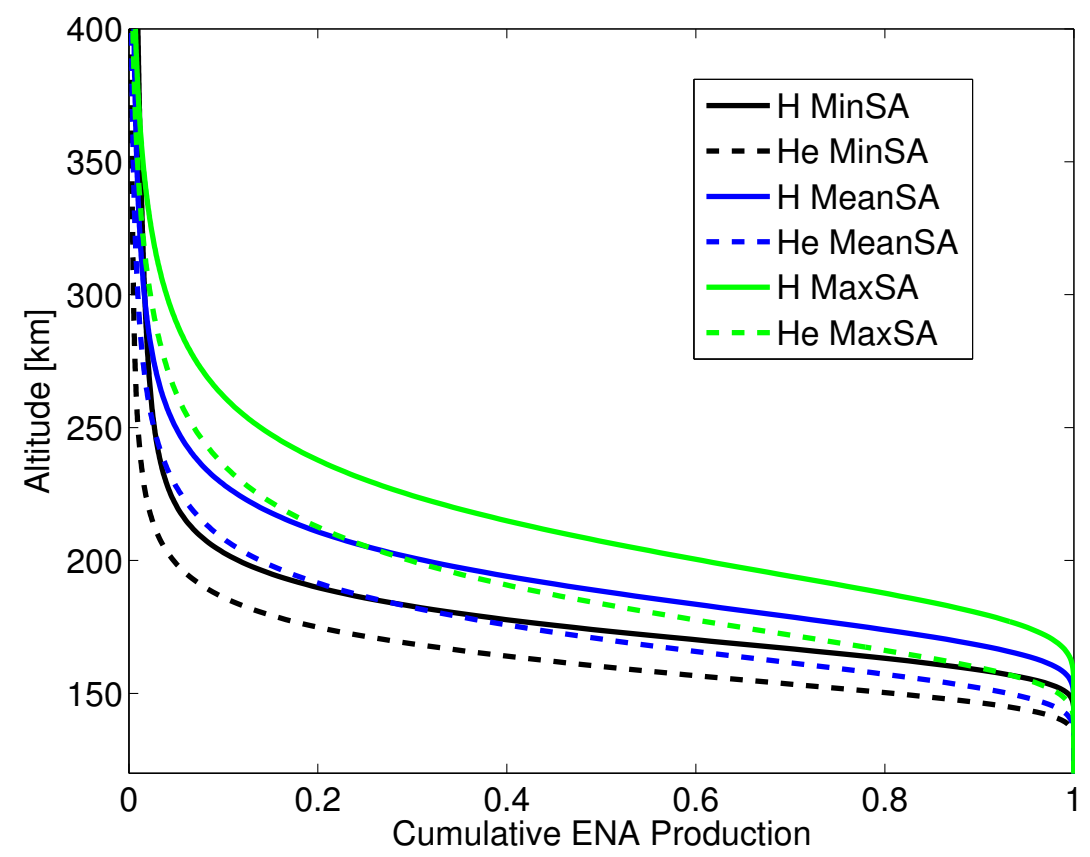

Fig. 2.29: Altitude dependent cumulative distribution function for nascent ENA production. Both hydrogen and helium ENAs are shown with solid and dashed lines for the three different Mars atmosphere models corresponding to minimum, mean, and maximum SA. Average SW energies of $1 \mathrm{keV}$ and $4 \mathrm{keV}$ for hydrogen and helium were used for calculation of production altitudes. 
using MC transport methods described in Section 2.2.

Several key parameters were extracted from the MC simulations to best display physical details of the thermalization and possible multi-collisions backscattering of incident ENAs as well as the production of upward and escape fluxes of secondary hot atoms and molecules in the atmosphere. Results are presented for realistic initial energy-altitude distributions using the different atmospheric density models fro minimum, mean, and maximum solar activity. In addition, results for mono-energetic ensembles of hydrogen atoms with an initial energy of $1 \mathrm{keV}$, and helium atoms with initial energy of $4 \mathrm{keV}$, are also included for comparison. The energies of the mono-energetic ensembles were chosen as they represent the most common energies associated with the model SW energy distribution. These mono-energetic ensembles of nascent ENAs were all initialized at an altitude of $200 \mathrm{~km}$, which is about the average alitutde of nascent ENA formation, and were transported using the mean solar activity neutral atmosphere model. Ensembles for hydrogen and helium ENAs whose collisions are described by the HS cross sections, were also considereed to compare with the anisotropic, quantum mechanical cross sections. HS cross sections have been obtained from Van der Waals radii used for the physical radii of all atoms and molecules [72]. These HS ensembles were initialized using the realistic energy-altitude ENA distributions and were transported throught the mean solar activity neutral model atmosphere. All ensembles contained 50,000 test particles which was double the number of test particles required for saturated statistics of all processes of interest.

Results were obtained from the simulations using discrete, 2D probability den- 
sities $f\left(x_{i}, y_{j}\right)$, for two parameters of interest, $x_{i}$ and $y_{j}$, where each test particle was placed in the appropriate discrete bin at every transport step. The probability densities are normalized such that

$$
\sum_{i, j} f\left(x_{i}, y_{j}\right) \Delta x \Delta y=1
$$

where $\Delta x$ and $\Delta y$ are the bin sizes for the $x$ and $y$ parameters. Average values for a given parameter were obtained using a weighted average

$$
<y_{j}>=\frac{\sum_{i} x_{i} f\left(x_{i}, y_{j}\right)}{\sum_{i} f\left(x_{i}, y_{j}\right)}
$$

where $\left\langle y_{j}\right\rangle$ is the weighted average value.

During the MC transport simulations, an ENA test particle is classified as being thermalized if all of the original energy of a few $\mathrm{keV} / \mathrm{amu}$ is transfered to other atmospheric particles during the simulation, thus making the test particle thermal with its environment. A formal cutoff energy of $0.1 \mathrm{eV}$ was used to determine if the test particles had thermalized. This energy was chosen as it is slightly above the mean thermal energy of hydrogen, $0.02 \mathrm{eV}$, and helium, $0.08 \mathrm{eV}$, at a typical temperature of $200 \mathrm{~K}$ found in the atmosphere of Mars [38].

Energy distributions of precipitating ENAs are extremely useful as they provide insight into the time-dependent thermalization process of the ensembles. Timedependent energy distributions of precipitating particles allow the evaluation of rates involved with ENA energy relaxation and atmospheric heating for different parameters of precipitating fluxes and upper atmosphere conditions of Mars. Fig. 2.30 displays energy distributions for the HS, mono-energetic, and realistic SW energy ensembles for 
both hydrogen and helium ENAs, all of which utilize the mean solar activity neutral atmosphere model. The HS ensembles shown in Fig. 2.30 lose the vast majority of their energy very quickly yet take more time to completely thermalize than the realistic SW or mono-energetic ensembles, both of which utilize quantum mechanical cross sections. The mono-energetic and realistic SW ensembles shown in Fig. 2.30 display how the helium ENAs lose their energy much slower than the hydrogen ENAs. In both of the realistic SW energy distributions ensembles, an interesting feature can be seen at times $0.29,0.37$, and 0.52 seconds which resembles the mixing of two fluxes with different speeds as the lower energetic portion of the ensembles thermalizes first, leading to a low energy peak in the distributions, followed by a higher energy peak which slowly merges with the first peak. This feature is not seen in either the HS of mono-energetic ensembles due to the fact that this is a feature of both small angle, anisotropic quantum mechanical cross sections used in these ensembles as well as initial energy distributions with a large spread in energies.

Fig. 2.31 displays the average energy of the test particle ensembles as a function of altitude in the Mars atmosphere for hydrogen and helium ENAs utilizing Eq. 2.82. An unexpected feature can be seen in Fig. 2.31 in that there is a steady decrease in average energy with decreasing altitude starting from $160 \mathrm{~km}$ as the ENAs penetrate into the atmosphere, yet there is an altitude for both hydrogen and helium ENAs at which the average energy begins to increase. Additional mono-energetic ensembles for hydrogen ENAs with energies of 0.5 and $2 \mathrm{keV}$ are shown in Fig. 2.31a to better understand the average energy increases for the realistic SW ensembles. These increases do not exist for 

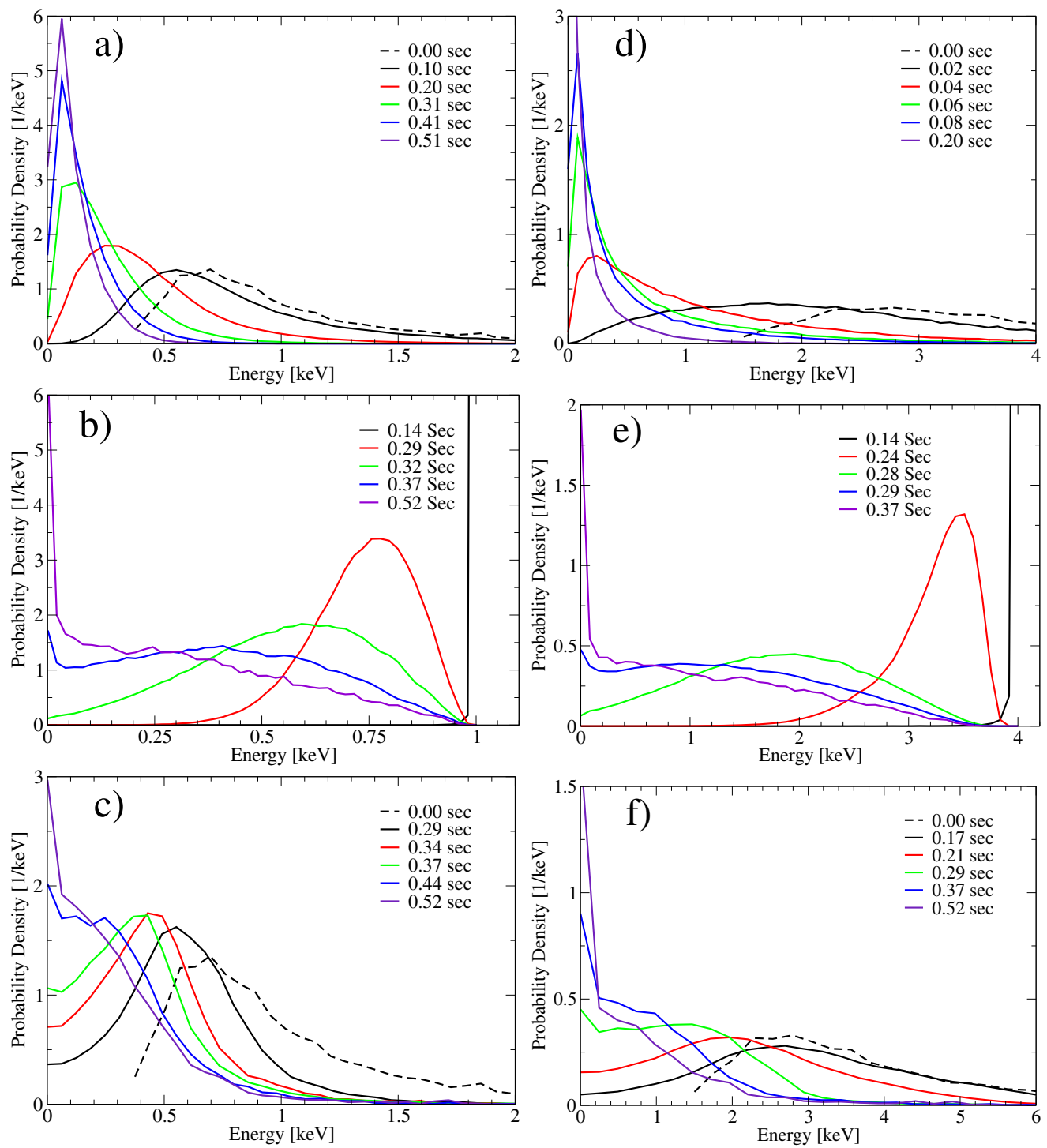

Fig. 2.30: Energy distributions for precipitating hydrogen ENAs are shown for HS cross sections a), mono-energetic $1 \mathrm{keV} \mathrm{b}$ ), and realistic SW energy c) ensembles, all utilizing the mean SA model atmosphere. Results are shown in a similar manner for precipitating helium ENAs in $\mathrm{d}-\mathrm{f}$ ) with the monoenergetic ensemble having an energy of $4 \mathrm{keV}$. 
mono-energetic ensembles which decrease monotonically with decreasing altitude and penetrate deeper into the atmosphere as the initial energy is increased. The altitude, at which the average energy begins to increase, is located at $100 \mathrm{~km}$ and $110 \mathrm{~km}$ for hydrogen and helium respectively and is attributed to the average penetration depths of the ENAs with realistic SW energies. This increase in average energy, along with a significant decrease in numbers of precipitating particles, can then be attributed to the fact that these low altitudes are only accessible to the most energetic portion of the initial ENA energy distribution and thus the total average energy of the ensemble jumps from including all particles to including only the most energetic ones. Fig. 2.31 also displays the differences in average energy due to different atmospheric neutral density models. These differences are small for altitudes less than $160 \mathrm{~km}$ yet begin to play a larger role for higher altitudes. For example, average energies differ by $50 \mathrm{eV}$ and 200 $\mathrm{eV}$ between minimum and maximum solar activity atmosphere models at an altitude of $200 \mathrm{~km}$ for hydrogen and helium ENAs respectively. In comparison to the realistic quantum mechanical cross sections, ensembles utilizing isotropic HS cross sections have large energy losses observed at higher altitudes, greater than 200 km, as seen in Fig. 2.31, resulting in a total penetration depth of $140 \mathrm{~km}$ and $130 \mathrm{~km}$ for hydrogen and helium ENAs, both of which are much lower than their quantum mechanical counterparts.

The altitude at which test particles thermalize with the atmosphere was also obtained from the MC simulations for all ensembles. Fig. 2.32 displays probability densities for the thermaliztion altitude of all ensembles. Very little difference in thermalization altitude can be seen in comparing the three different atmosphere models with all models 

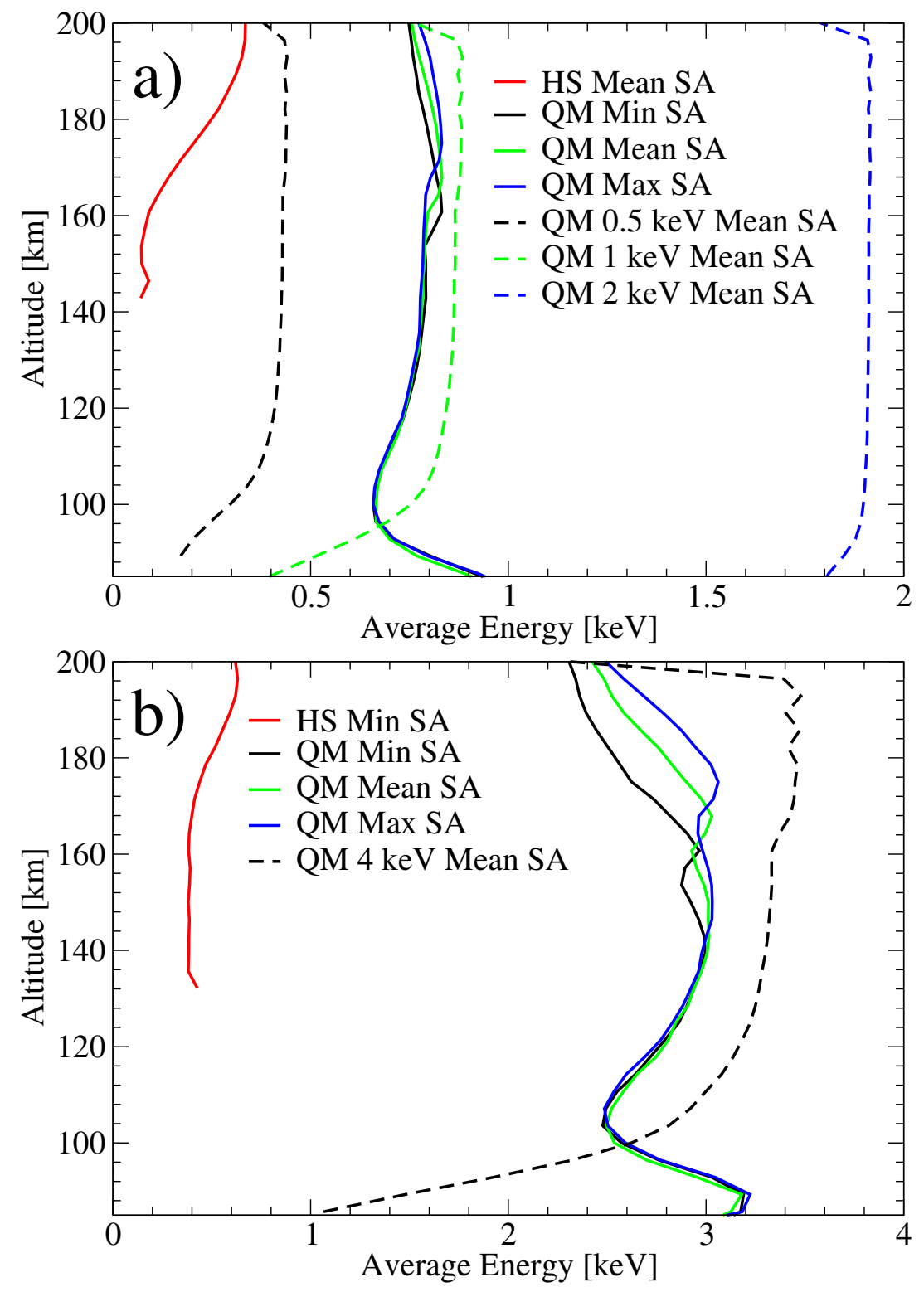

Fig. 2.31: Average weighted energy for both hydrogen a) and helium b) ENAs as a function of altitude for all three atmosphere models. Additionally, ensembles utilizing HS cross sections and mono-energetic ensembles are also shown for comparison. 
having the highest thermalization probability at $95 \mathrm{~km}$ and $98 \mathrm{~km}$ for hydrogen and helium respectively. Drastic differences between the HS ensembles and the quantum mechanical ensembles can be seen in Fig. 2.32 with the highest thermalization probability occurring at $160 \mathrm{~km}$ for both hydrogen and helium HS ensembles. The probability distribution for the helium ensemble using HS cross sections is wider than its hydrogen counterpart, being due to higher collision rates for the helium ENAs with larger HS radii [72]. The mono-energetic distributions have slightly deeper thermalization depths and narrower distributions than the SW energy distribution ensembles, demonstrating the collective effects of having an initial energy distribution as compared with a single initial energy.

In addition to thermalization altitudes, thermalization times were also obtained from the MC simulations for all ensembles. Thermalization time probability densities for all ensembles are shown in Fig. 2.33. Slightly larger average scattering angles for a given ENA velocity, shown in Fig. 2.28, leads to faster thermalization of helium ENAs as compared to hydrogen ENAs, an effect which is clear from Fig. 2.33. The monoenergetic probability distributions are narrower than both of their respective realistic SW energy distribution ensembles, yet the tails of all ensemble distributions decay in a very similar manner starting at 0.5 seconds. The thermalization time probability density for HS cross sections in Fig. 2.33 is vastly different from the quantum mechanical ensembles, with average thermalization times an order of magnitude larger than their quantum mechanical counterparts. These large thermlization times are attributed to the test particles in the HS ensembles occupying only high altitude layers of the atmo- 

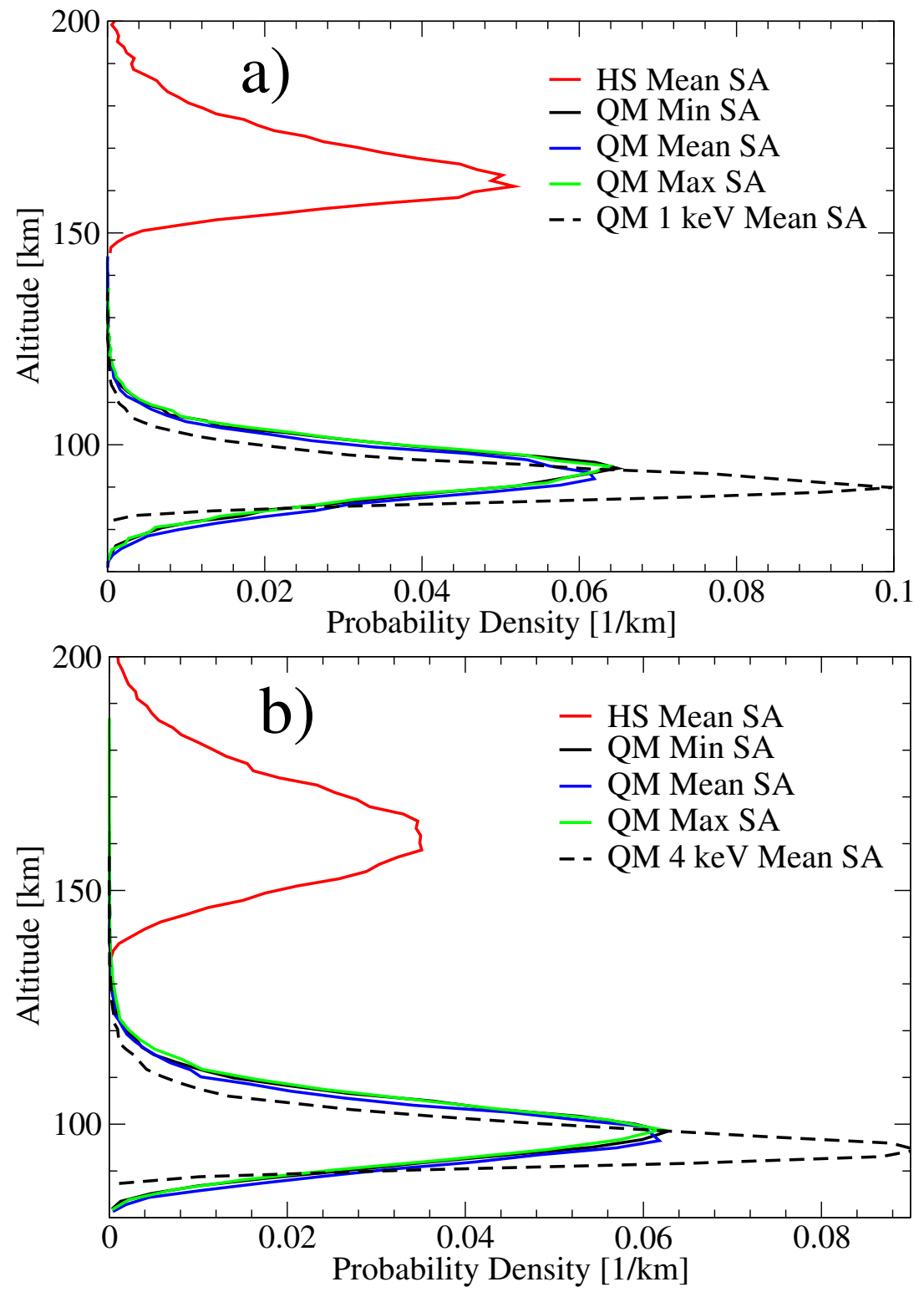

Fig. 2.32: Thermalization altitude probability densities for both hydrogen a) and helium b) ENAs using all three atmosphere models. Additionally, ensembles utilizing HS cross sections and mono-energetic ensembles are also shown for comparison. 
sphere, above $150 \mathrm{~km}$, as seen in Figs. 2.31 and 2.32. The high altitude atmosphere layers have significantly smaller neutral densities as compared with lower atmosphere layers where the ensembles of test particles utilizing quantum mechanical cross sections thermalize. For example, the neutral density increases by 3-4 orders of magnitude from $150 \mathrm{~km}$ to $100 \mathrm{~km}$ [38]. The average mean free path for HS ensembles within the layer of atmosphere between 150-200 km, where the majority of the ENAs using HS cross sections thermalize, ranges from several to tens of kms. These large mean free paths lead to long transport times between collisions, in particular, ENAs with energies less than $20 \mathrm{eV}$ are transported from 0.1 seconds or more on average. The combination of these long transport times between collisions and the hundreds of collisions required before thermalization results in the long thermalization times shown in Fig. 2.33 for ensembles utilizing HS cross sections.

During the transport and thermalization of the ENA test particles, several thousand collisions occur with the atmospheric gases, the vast majority of which happen with very small scattering angles, thus transferring to the ambient gas a very small amount of energy as determined by Eq. 2.64. With so many collisions occurring during the thermalization process, several low probability, large-angle scattering events transpire during the lifetime of the test particle which provide enough energy to the target particle for it to be considered hot itself. The energy transfer threshold to be considered a secondary hot atom/molecule was set to $0.1 \mathrm{eV}$ which is significantly higher than atmospheric thermal energies.

Production yields of secondary hot atoms and molecules per precipitating particle 

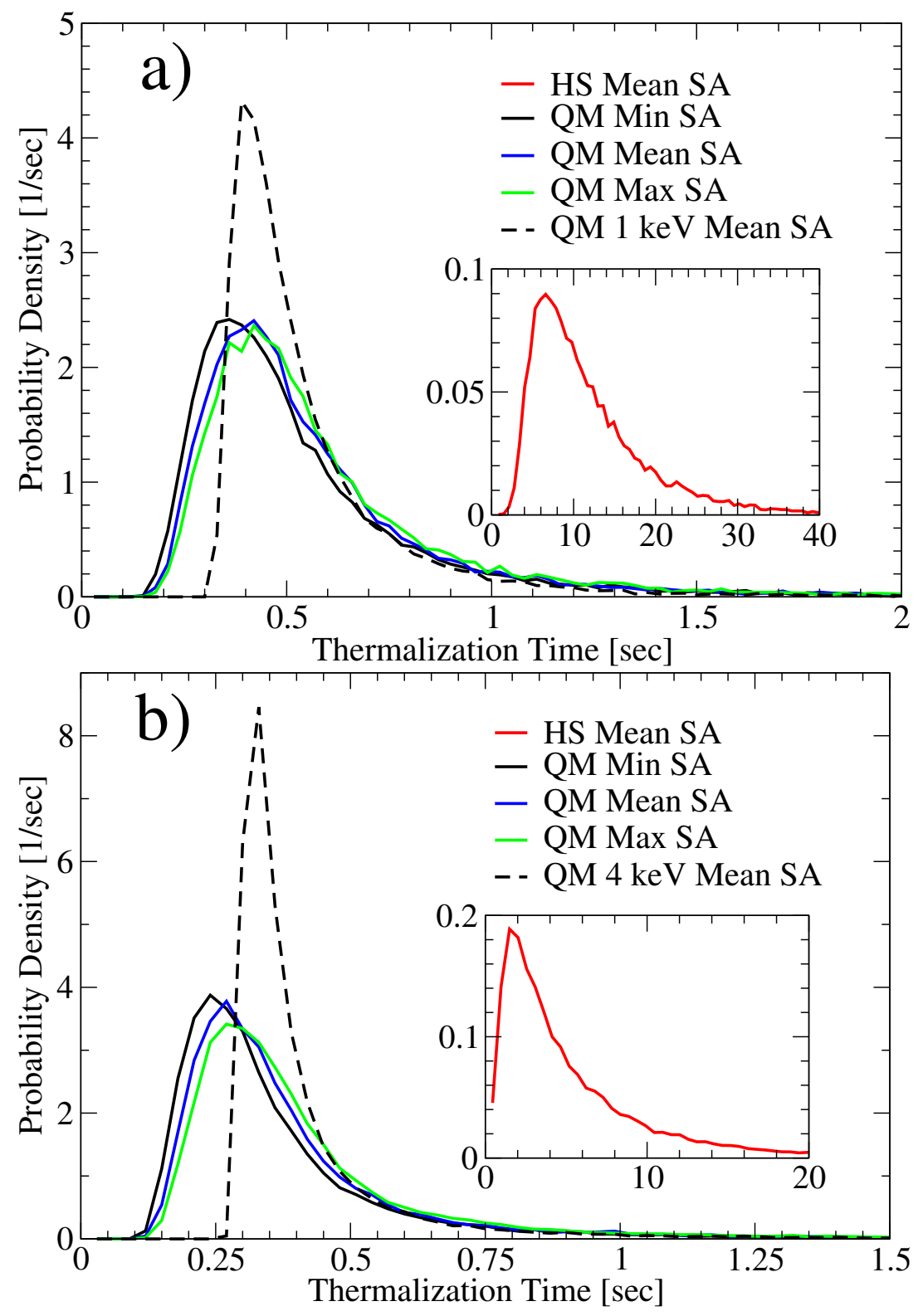

Fig. 2.33: Thermalization time probability densities for both hydrogen a) and helium b) ENAs using all three atmosphere models as well as ensembles utilizing HS cross sections and mono-energetic ensembles. Results from ensembles utilizing hard sphere cross sections are shown in the inset plots as the thermalization times for these ensembles were much larger than ensembles utilizing quantum cross sections. 
were calculated as a function of altitude as

$$
Q(z)=\frac{N_{S H}(z)}{N \Delta z}
$$

where $N$ is the total number of incident ENAs, $N_{S H}(z)$ is the total number of nascent secondary hot atoms/molecules created in the atmosphere layer at altitude $z$ with layer thickness $\Delta z$. Fig. 2.34 displays the secondary hot atom/molecule production yields for the major atmospheric species as a function of altitude. Additionally, to illustrate the dependence of production yields on solar conditions, Fig. 2.34 shows production yields induced by both hydrogen and helium ENAs for all three neutral atmosphere models. For all atmospheric atoms and molecules, the profile shape for nascent secondary hot atom/molecule production yields look very similar with maximum production occurring deep int he atmosphere around $80 \mathrm{~km}$. For both hydrogen and helium ENAs incident on all atmospheric density models, the production yields for nascent secondary hot $\mathrm{H}$, $\mathrm{H}_{2}$, and He are significantly lower than for other atmospheric species due to their small relative densities.

In addition to altitude dependent production yields, normalized energy distributions for nascent secondary hot atoms and molecules were also calculated. The energy distribution for nascent secondary hot atoms and molecules mostly reflect the energytransfer processes of keV collisions. Because of this, the secondary hot energy distributions were found to be ear identical for the different atmosphere models and altitudes, and thus are presented in Fig. 2.35 as independent of altitude and for the mean solar activity atmosphere model only. The nascent energy distribution for secondary hot atoms and molecules induced by helium ENAs is peaked at a slightly higher energy, 0.7 

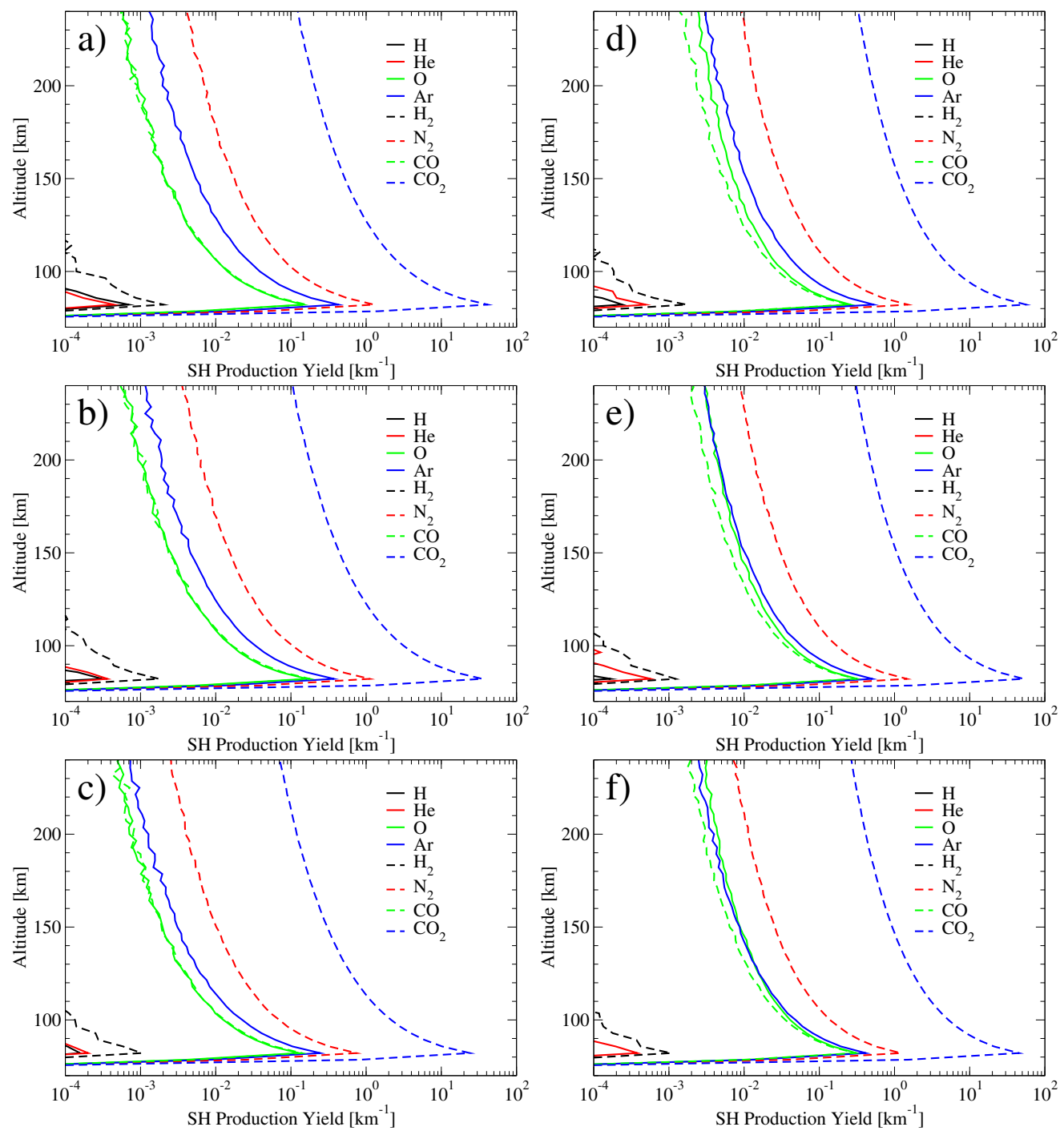

Fig. 2.34: $\mathrm{SH}$ atomic and molecular production rates due to precipitating hydrogen ENAs using the three atmosphere models for minimum a), mean b), and maximum SA c). The same data is shown for precipitating helium ENAs in figures $d-f)$. 
$\mathrm{eV}$, as compared with the hydrogen ENAs, which is peaked at $0.5 \mathrm{eV}$. These differences in the energy peaks are due in part by the larger average scattering angles of helium ENAs, Fig. 2.28, as well as the mass ratio in Eq. 2.64 favoring heavier projectiles for more energy transferred during a given collision. To determine the fraction of nascent secondary hot atoms and molecules which have an energy above their respective escape energy, the distribution in Fig. 2.35 were integrated starting from the escape energy of the atom or molecule. Table 2.7 shows the escape energies of the secondary hot atoms and molecules at $700 \mathrm{~km}$ as well as the percentage of nascent secondary hot atoms and molecules, created by incident hydrogen and helium ENAs, which have energies above their respective escape energy. Secondary hot $\mathrm{Ar}$ and $\mathrm{CO}_{2}$ do not have any realistic probability to escape due to their high masses and thus high escape energies, as shown in Table 2.7.

Using the nascent secondary hot atomic and molecular production yields along with the nascent energy distributions, secondary hot atomic and molecular escape fluxes were estimated. Nascent secondary hot atomic and molecular velocity directions were observed to be uniform across all simulations, so we assume initial velocity distributions are isotropic. These nascent isotropic distributions were used along with a simplified collision transparency formalism to estimate secondary hot atomic and molecular escape fluxes induced by precipitating hydrogen and helium ENAs using the mean solar activity atmosphere model. In this simplified formalism, the ratio of escaping secondary hot 

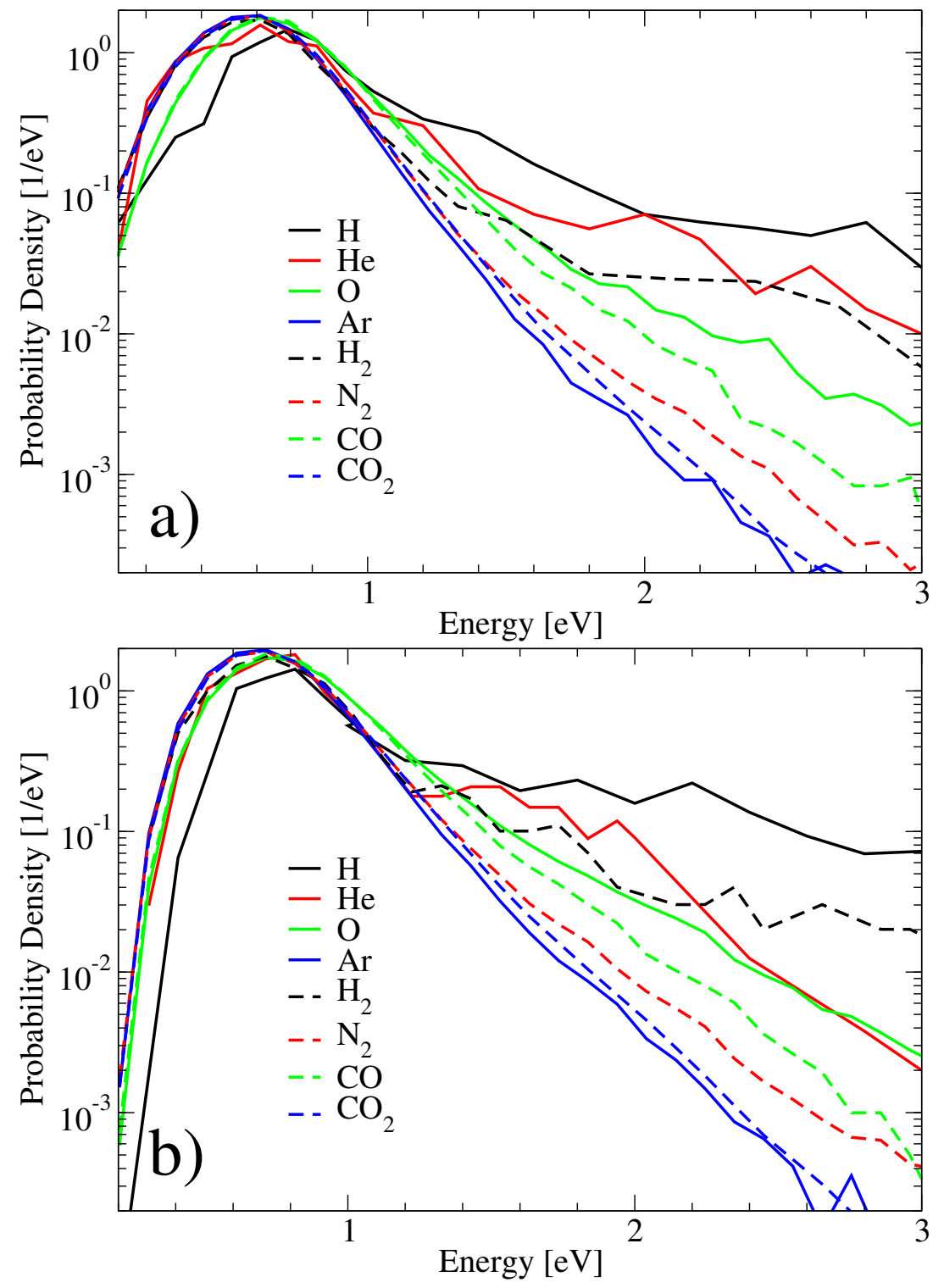

Fig. 2.35: Nascent $\mathrm{SH}$ atomic and molecular energy distributions, normalized to unity, induced by collisions with hydrogen a) and helium b) ENAs using the mean SA atmosphere model. 
Table 2.7: Percentage of nascent secondary hot atoms and molecules capable of escaping. Atomic and molecular escape energies $E_{\text {esc }}$ calculated at an altitude of $700 \mathrm{~km}$.

\begin{tabular}{|c|ccc|}
\hline & $\mathbf{E}_{e s c}(\mathrm{eV})$ & H ENA $(\%)$ & He ENA $(\%)$ \\
\hline $\mathrm{H}$ & 0.11 & 100 & 100 \\
$\mathrm{He}$ & 0.44 & 80.18 & 93.75 \\
$\mathrm{O}$ & 1.8 & 1.94 & 3.34 \\
$\mathrm{Ar}$ & 4.4 & 0 & 0 \\
$\mathrm{H}_{2}$ & 0.22 & 93.55 & 100 \\
$\mathrm{~N}_{2}$ & 3.0 & 0.01 & 0.02 \\
$\mathrm{CO}$ & 3.0 & 0.06 & 0.06 \\
$\mathrm{CO}_{2}$ & 4.8 & 0 & 0 \\
\hline
\end{tabular}

atomic or molecular fluxes, $\Phi_{e s c}$, to incident ENA fluxes, $\Phi_{i n c}$, may be written

$$
\frac{\Phi_{\text {esc }}}{\Phi_{\text {inc }}}=\frac{(1+\alpha)}{2} \int_{z_{\min }}^{z_{\max }} d z Q(z) \int_{\epsilon_{\text {esc }}}^{\infty} d \epsilon \rho(\epsilon) \int_{\sqrt{\frac{\epsilon_{\text {esc }}}{\epsilon}}}^{1} d u T(u, z, \epsilon)
$$

where secondary hot atomic and molecular productions, $Q(z)$, are integrated over the altitude height, nascent energy distributions, $\rho(\epsilon)$, are integrated from the respective escape energy to infinity, and a conical component of the isotropic velocity distribution is integrated over a newly defined variable $u \equiv \cos \theta$, with $\theta$ being the standard polar angle, such that all velocity directions with upward components greater than escape velocity are considered. The integral over the upward escape velocity cone also includes a collision transparency factor, $T(u, z, \epsilon)$, which gives the escape probability for a particle 
with velocity in the $u$ direction at altitude $z$ with energy $\epsilon$ and is defined as

$$
T(u, z, \epsilon)=\exp \left(-\frac{1}{u} \int_{z}^{z_{\max }} \sum_{i} n_{i}\left(z^{\prime}\right) \sigma_{i}^{D}(\epsilon) d z^{\prime}\right)
$$

where $n_{i}(z)$ is the density of the $i^{t h}$ atmospheric species at altitude $z$ and $\sigma_{i}^{D}(\epsilon)$ is the momentum-transfer cross sections between the secondary hot atom or molecule and the $i^{\text {th }}$ atmospheric species. The momentum-transfer (diffusion) cross section is defined using the differential cross section $|f(\epsilon, \theta)|^{2}$ as

$$
\sigma^{D}(\epsilon)=\int(1-\cos \theta)|f(\epsilon, \theta)|^{2} d \Omega
$$

and is commonly used to describe processes involving energy transport [73,74,21]. The diffusion cross sections were used in Eq. 2.85 instead of total cross sections as they filter ultra small scattering angles which effectively transfer no energy to the target atoms [21]. The factor $\alpha$ in Eq. 2.84 describves the fraction of nascent secondary hot particles which have downward velocities and yet are reflected upward from the more dense gas layers due to large angle collisions. These upwared reflections may contributed to the secondary hot atomic and molecular escape fluxes. While these reflected secondary hot particles may have different energy distributions than those shown in Fig. 2.35, they are included since large reflection coefficients were observed for the incident $\mathrm{keV} / \mathrm{amu}$ ENAs. The reflection coefficients found range from 0.19 to 0.47 depending on the projectile ENA and the atmopheric condition. For this work, the constant $\alpha=0.2$ was used as it is the lower rnage of the reflection coefficients found and is a conservative estimate of the actual reflection value.

The MC methods of Section 2.2 were used to determine escape probabilities for secondary hot helium created from precipitating hydrogen ENAs in the mean solar 
activity model atmosphere as a way to compare with the transparency formalism of Eq. 2.84. Using 50,000 test particles with MC methods, the escape ratio was found to be $0.0170 \%$ as compared to $0.0102 \%$ calculated using Eq. 2.84 with $\alpha=0.2$. The transparency escape ratio differs by a percent error of $40 \%$ from the MC result making the simple transparency formalism described above a viable alternative to the timeexpansive full $\mathrm{MC}$ calculation. With the comparison made between $\mathrm{MC}$ and collision transparency methods in good agreement, escape fluxes for all secondary hot atomic and molecular species are shown with utilization of the collision transparency method.

Realistic secondary hot atomic and molecular escape fluxes were estimated using an average total incident ENA flux of $\Phi_{\text {inc }}^{\text {tot }}=9.41 \times 10^{7} \mathrm{~cm}^{-2} \mathrm{~s}^{-1}$, calculated using an average SW speed of $437 \mathrm{~km} / \mathrm{s}$ [9] and an average SW ion density of $5 \mathrm{~cm}^{-3}$ at $1 \mathrm{AU}$ [71]. An average SW helium component of $2 \%$ [43] was used to determine the individual fluxes of precipitating hydrogen, $\Phi_{i n c}^{H}=9.22 \times 10^{7} \mathrm{~cm}^{-2} \mathrm{~s}^{-1}$, and helium, $\Phi_{i n c}^{\mathrm{He}}=1.88 \times$ $10^{6} \mathrm{~cm}^{-2} \mathrm{~s}^{-1}$. Table 2.8 displays the escape probabilities calculated with Eq. 2.84 as well as estimated escape fluxes using the incident SW ion fluxes above. $\mathrm{H}$ non-thermal escape fluxes are included in Table 2.8, although for the Mars atmosphere escape of $\mathrm{H}$ atoms are governed by the Jeans thermal escape. In comparison, escape fluxes of neutral helium, due to collisions with hot oxygen created in dissociative recombination of $\mathrm{O}_{2}^{+}$, were calculated to be $1.6 \times 10^{6} \mathrm{~cm}^{-2} \mathrm{~s}^{-1}$ at minimum solar activity [21]. Oxygen escape fluxes, again due to dissociative recombination, were calculated to range from $10^{7}$ to $10^{8} \mathrm{~cm}^{-2} \mathrm{~s}^{-1}$ at minimum solar activity using several different collisional models $[36,2]$. The escape fluxes estimated from the mechansim of SW ion precipitation are 
Table 2.8: Secondary hot atomic and molecular escape probabilities and escape fluxes. Secondary hot escape probabilities were calculated using Eq. 2.84 while estimated escape fluxes were obtained using incident ENA fluxes of $\Phi_{i n c}^{H}=$ $9.22 \times 10^{7} \mathrm{~cm}^{-2} \mathrm{~s}^{-1}$ and $\Phi_{i n c}^{H e}=1.88 \times 10^{6} \mathrm{~cm}^{-2} \mathrm{~s}^{-1}$. Total escape probabilities and fluxes are shown in the last row for all secondary hot atomic and molecular species.

\begin{tabular}{|c|c|c|c|c|}
\hline \multirow{2}{*}{} & \multicolumn{2}{|c|}{ H ENA } & \multicolumn{2}{c|}{ He ENA } \\
\cline { 2 - 5 } & $\frac{\Phi_{e s c}}{\Phi_{i n c}}$ & $\Phi_{e s c}\left(\frac{1}{c^{2} s}\right)$ & $\frac{\Phi_{e s c}}{\Phi_{i n c}}$ & $\Phi_{e s c}\left(\frac{1}{c m^{2} s}\right)$ \\
\hline $\mathrm{H}$ & $4.54 \mathrm{e}-4$ & $4.18 \mathrm{e}+4$ & $9.42 \mathrm{e}-5$ & $1.79 \mathrm{e}+2$ \\
$\mathrm{He}$ & $1.02 \mathrm{e}-4$ & $9.43 \mathrm{e}+3$ & $4.83 \mathrm{e}-4$ & $9.17 \mathrm{e}+2$ \\
$\mathrm{O}$ & $5.57 \mathrm{e}-4$ & $5.12 \mathrm{e}+4$ & $3.62 \mathrm{e}-3$ & $6.87 \mathrm{e}+3$ \\
$\mathrm{Ar}$ & 0 & 0 & 0 & 0 \\
$\mathrm{H}_{2}$ & $8.13 \mathrm{e}-4$ & $7.48 \mathrm{e}+4$ & $1.52 \mathrm{e}-3$ & $2.89 \mathrm{e}+3$ \\
$\mathrm{~N}_{2}$ & $1.04 \mathrm{e}-5$ & $9.53 \mathrm{e}+2$ & $3.62 \mathrm{e}-5$ & $6.87 \mathrm{e}+1$ \\
$\mathrm{CO}$ & $7.40 \mathrm{e}-6$ & $6.81 \mathrm{e}+2$ & $2.23 \mathrm{e}-5$ & $4.23 \mathrm{e}+1$ \\
$\mathrm{CO}$ & 0 & 0 & 0 & 0 \\
\hline $\mathrm{ALL}$ & $1.94 \mathrm{e}-3$ & $1.79 \mathrm{e}+5$ & $5.77 \mathrm{e}-3$ & $1.10 \mathrm{e}+4$ \\
\hline
\end{tabular}

roughly three orders of magnitude smaller than the fluxes estimated due to hot oxygen generated in dissociative recombination of $\mathrm{O}_{2}^{+}$, yet still may be important in estimating long term atmospheric mass losses, especially taking into account intensive fluxes of SW plasma during earlier periods in the Sun's history.

In addition to thermalizing and inducing secondary hot atomic and molecular escape fluxes, the ENA test particles may also escape the atmosphere if they reach a 
height of $700 \mathrm{~km}$ with an upward velocity and an energy above their respective escape energy. Although all ensembles studied in this work are initialized directly incident to the surface of the planet with solar zenith angles equal to $0^{\circ}$, even for this case a significant percnetage of all ensembles is reflected back upwards and escapes the planet.

Table 2.9 displays details of initial conditions for the different ensembles of precipitating particles used in our simulations as well as the reflection probability, average energy of reflected ENAs and average number of collisions before being reflected for each ensemble. In addition to Table 2.9, Fig. 2.36 displays the energy distributions for the reflected ENAs. For easy comparison, the initial incident energy distribution is shown, normalized to unity, for both hydrogen and helium ENAs in Fig. 2.36 while the reflected energy distributions for all ensembles are normalized to their respective reflection probability, $P_{R}$, in Table 2.9. Fig. 2.36 demonstrates how the reflected energy distributions are all shifted to lower energies due to the energy loss required from several large angle collisions to reflect the incident particles upward. The energy distributions from Fig. 2.36 also show how energetic the back reflected particles are, with the majority of escaping hydrogen having energies of $700 \mathrm{eV}$ and reflected helium having energies of $2.3 \mathrm{keV}$. Our results for reflected hydrogen ENAs are similar to those previously reported where it was observed that the reflection probability was 0.58 [67]. Additionally, the reflected hydrogen ENAs were reported to have an average energy of $470 \mathrm{eV}$ which is more than half of their average initial energy of $800 \mathrm{eV}$ [67]. This signifies that if a particle does get reflected, it occurs quickly after becoming an ENA, before it gets very deep into the atmosphere where the transparency becomes low. This effect an be 
Table 2.9: Energetic neutral atom reflection statistics and ensemble parameters for collisional cross sections (CS) utilized, either HS (HS) or quantum mechanical (QM), initial energy, $\mathrm{E}_{0}$, initial altitude, $\mathrm{z}_{0}$, and the solar activity (SA) Mars atmosphere model used during transport. PD is shown for parameters which utilized probability distributions for initial conditions. Ensemble averages for ENA reflection probability, $\mathrm{P}_{R}$, reflected energy, $\mathrm{E}_{R}$, and number of collisions before being reflected from the atmosphere, $\mathrm{N}_{R}$, are also

shown.
\begin{tabular}{|c|c|c|c|c|c|c|c|}
\hline & CS & $\mathbf{E}_{0}$ & $\mathbf{z}_{0}$ & $\mathbf{S A}$ & $\mathbf{P}_{R}$ & $\mathbf{E}_{R}(\mathbf{e V})$ & $\mathbf{N}_{R}$ \\
\hline & HS & PD & PD & Mean & 0.43 & 264 & 53 \\
& QM & $1 \mathrm{keV}$ & $200 \mathrm{~km}$ & Mean & 0.51 & 773 & 7549 \\
& QM & PD & PD & Min & 0.47 & 767 & 10989 \\
& QM & PD & PD & Mean & 0.41 & 765 & 11091 \\
& QM & PD & PD & Max & 0.23 & 766 & 11003 \\
\hline \multirow{5}{*}{ He } & HS & PD & PD & Mean & 0.14 & 245 & 66 \\
& QM & 4 keV & $200 \mathrm{~km}$ & Mean & 0.23 & 2323 & 4757 \\
& PD & PD & Min & 0.24 & 2388 & 4906 \\
& PD & PD & Mean & 0.22 & 2398 & 4835 \\
& QM & PD & PD & Max & 0.19 & 2384 & 4793 \\
\hline
\end{tabular}


seen clearly from the mono-energetic ensembles in Fig. 2.36 which show peaks in the escaping energy probability around their initial energies of 1 and $4 \mathrm{keV}$. The HS ensembles are also informative as they display reflected energy distributions with significantly lower energies as compared to their quantum mechanical counterparts. The atmosphere model used for the different ensembles also displays different escape probabilities, with the minimum solar activity model having the highest escape probabilities and the maximum solar activity models having the lowest. Differences in the energy distributions of reflected ENAs may be very useful for future missions to Mars as a mechanism for determining parameters of the upper atmosphere.

Precipitation of ENAs, produced in the interaction between SW ions and atmospheric gas, has been investigated for the Mars atmosphere at different solar conditions. For an accurate description of the energy relaxation process, the parameters for accurate descriptions of energy-momentum transfer in atomic and molecular collisions have been developed using both quantum mechanical methods and empirical models. Properties of ENAs, originating in the interaction between the SW ions and atmospheric gas, were calculated for the upper atmosphere of Mars using neutral atmosphere models for minimum, mean, and maximum solar activity. MC simulations were constructed to transport nascent ENAs through the Martian atmosphere to determine properties of energy transfer, thermalization, production of secondary hot atoms and molecules, and reflection characteristics. Time-dependent energy distributions were obtained in addition to thermalization altitudes and atmospheric heating profiles. Production rates and energy distributions for secondary hot atoms and molecules were extracted and 

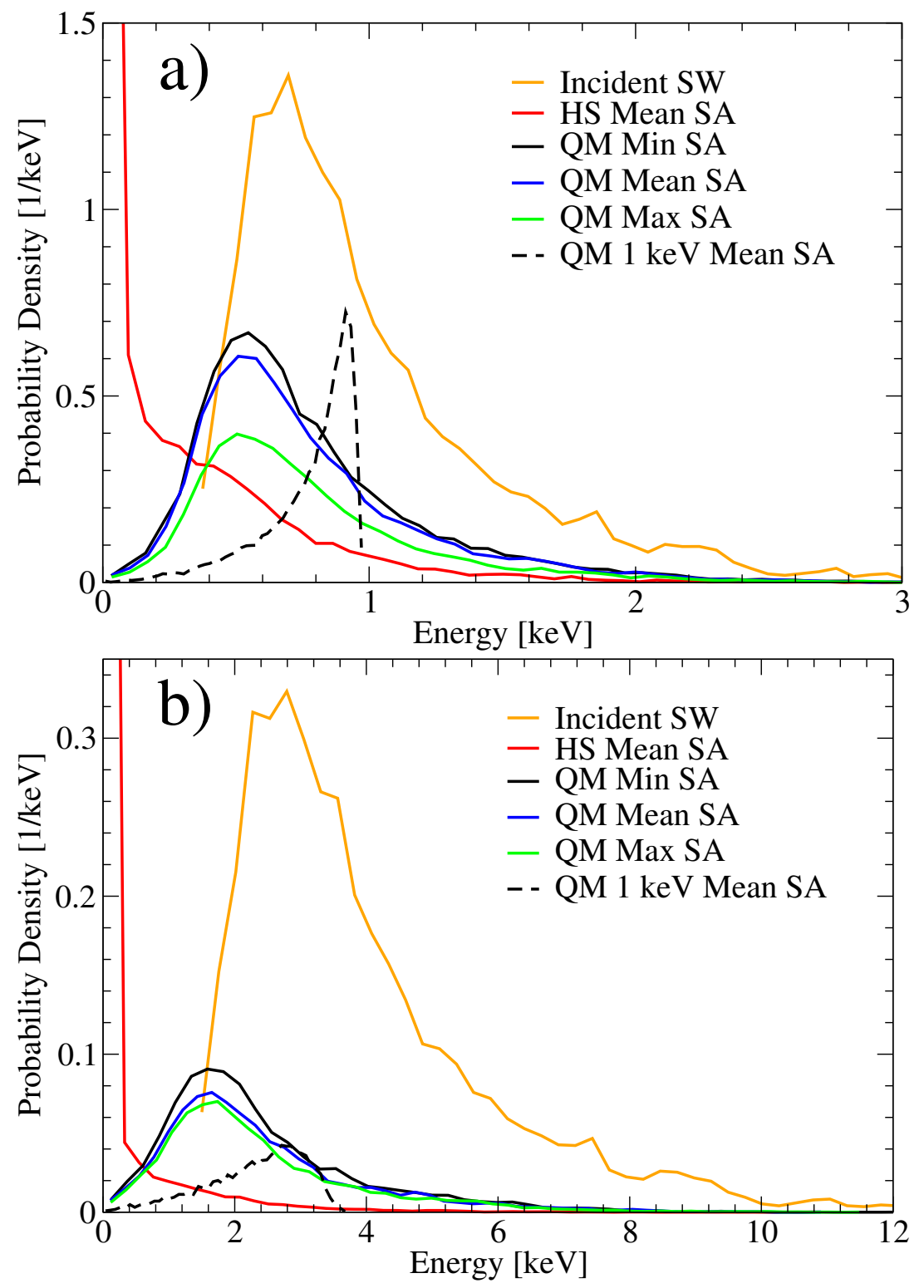

Fig. 2.36: Energy distributions for hydrogen a) and helium b) ENAs reflected by the atmosphere of Mars. The incident energy distributions are shown, normalized to unity, as well as the reflected energy distribution for all ensembles studied in this work. 
utilized to determine induced atomic and molecular escape fluxes from Mars. The information obtained from our MC simulations demonstrates the need for accurate, energy-angular dependent cross sections in modeling the energy relaxation, sputtering, and escape processes in planetary atmospheres as results obtained varied significantly between ensembles utilizing accurate cross sections and those which utilized isotropic, HS cross sections.

\subsection{Transport of Energetic Neutral Atoms in the Local Interstellar Medium}

In October of 2008, the Interstellar Boundary Explorer (IBEX) satellite was launched with the mission of investigating the interaction between solar wind (SW) ions and the interstellar medium at the edge of our solar system through imaging of energetic neutral atoms (ENAs) [75]. Since its launch, IBEX has provided several all-sky maps of ENA fluxes over the energy interval from $0.2-4.3 \mathrm{keV}$ [76,77]. Fig. 2.37 shows all sky maps for five high energy ENA energy bands from three complete sky mappings in the reference frame of IBEX using Mollweide projections [77]. The three mappings in Fig. 2.37 were collected 6 months apart on the opposite side of the Earth's orbit around the Sun. Additionally, Fig. 2.38 shows all sky maps taken from the low energy ENA instrument on IBEX. Interesting ribbons of intense ENAs can be seen in all mappings shown in Figs. 2.37 and 2.38. The cause of these intense ribbons is not well known, but a connection to the local interstellar magnetic field lines is most likely, directing flows of both solar and extrasolar ions until charge exchange (CX) occurs, producing nascent 
ENAs. Our aim is to investigate the possibility of a diffuse background of ENAs which originated as extra solar ions.

Investigation into a potential diffuse background of extra solar ENAs requires detailed knowledge of the local environment surrounding the Sun. The Sun moves around the center of the galaxy at an average speed of $230 \mathrm{~km} / \mathrm{s}$, making a complete orbit of a galactic year in 225-250 million Earth years [78]. During this galactic year, the local environment of the Sun may change drastically. Currently, our solar system lies within a warm cloud which extends several light years (LYs) in all directions and consists of partially ionized gas called the local interstellar cloud (LIC) [46]. The LIC has an average temperature of $6,000 \mathrm{~K}$ with extremely low neutral and ion densities of $0.2093 \mathrm{~cm}^{-3}$ and $0.0656 \mathrm{~cm}^{-3}$ respectively $[45,46,3]$. With these low neutral and ion densities, ENAs, created through CX collisions between stellar wind ions and neutral gases in the LIC, may be transported extremely far distances, on the order of LYs, before thermalizing with the LIC. The LIC is embedded in an even larger structure called the local bubble (LB) which is an extremely hot, $10^{6} \mathrm{~K}$ cavity which extends through the disc of the galaxy [79]. The LB is completely ionized with densities of 0.005 $\mathrm{cm}^{-3}$ and $0.0001 \mathrm{~cm}^{-3}$ for hydrogen and helium ions respectively [45,46,3]. Fig. 2.39 displays a neutral atom density map taken in the galactic plane [79]. Within the scale of Fig. 2.39 the LIC barely visible, extending a mere 2 parsecs (pcs), $1 \mathrm{pc}=3.26 \mathrm{LYs}$, from the origin.

Two different ENA sources are examined in this work as contributing to a local ENA background. The first of these ENA sources is stellar wind from nearby stars 


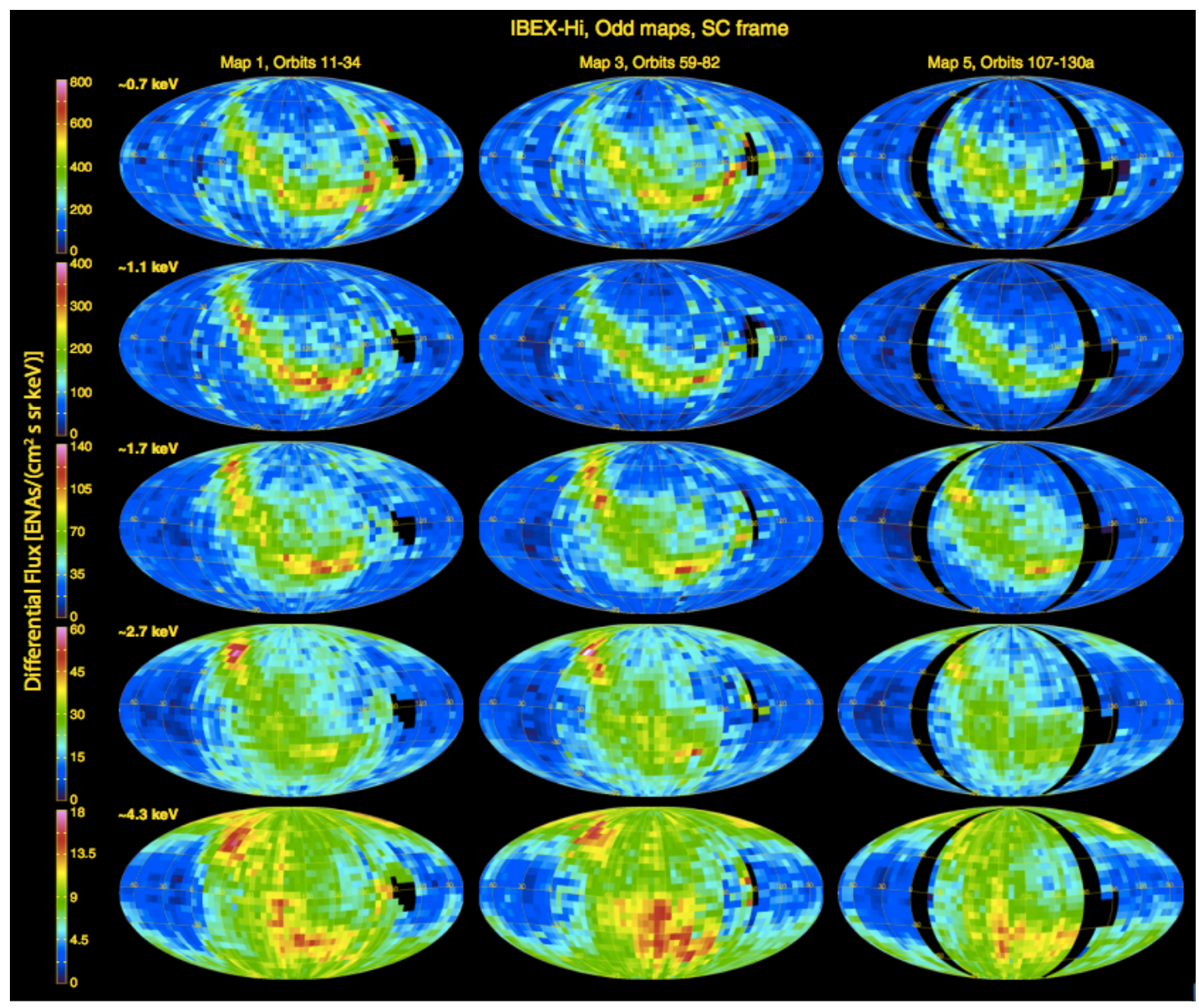

Fig. 2.37: IBEX all sky maps taken with the high energy instrument for energetic neutral atoms with energies of $0.7,1.1,1.7,2.7$, and $4.3 \mathrm{keV}$ in the space craft frame of reference [77]. Three complete all sky maps are shown for each energy, each taken 6 months apart. 


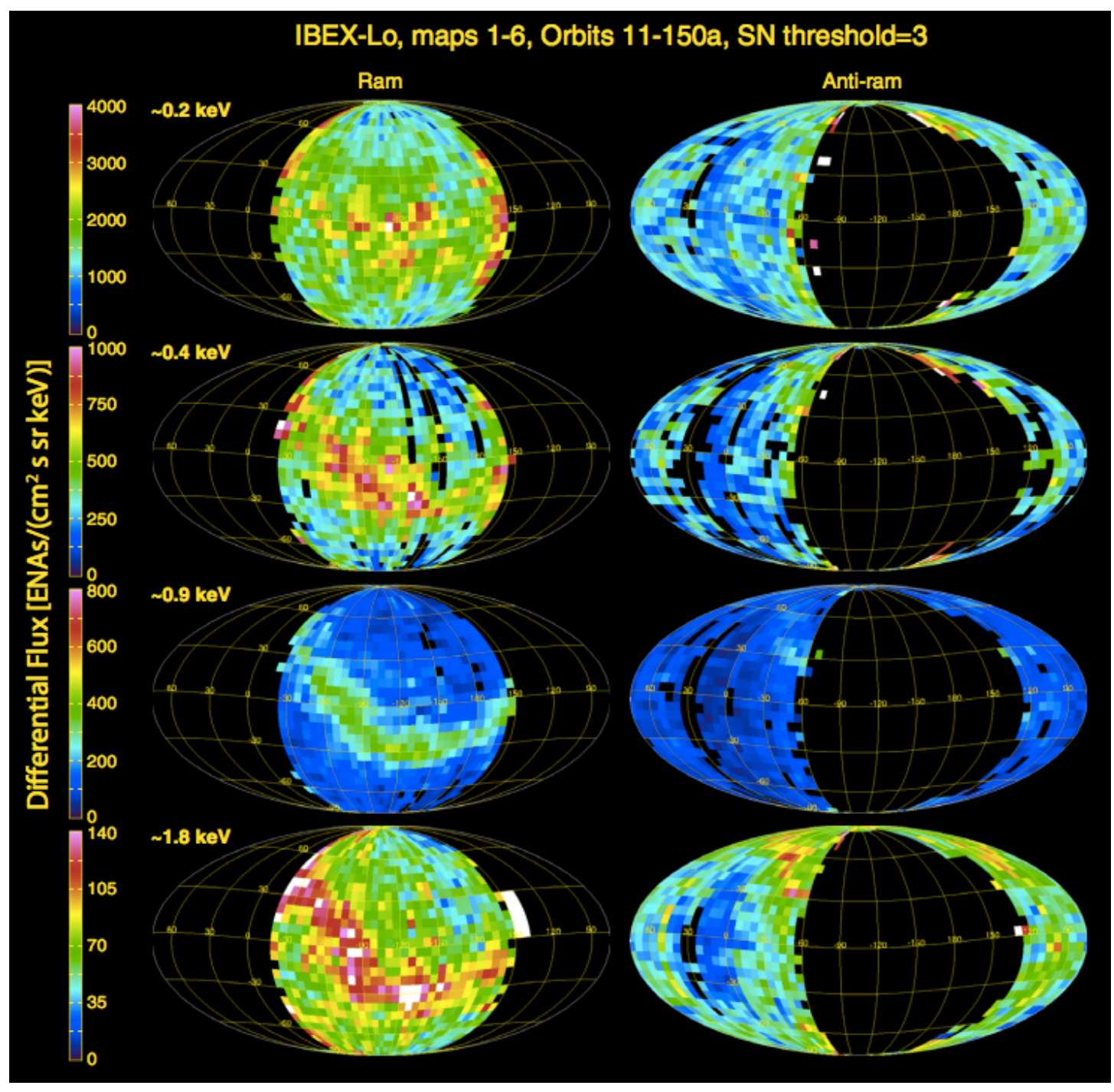

Fig. 2.38: IBEX all sky maps taken with the low energy instrument for energetic neutral atoms with energies of $0.2,0.4,0.9$, and $1.8 \mathrm{keV}$ in the space craft frame of reference [77]. The maps are separated to display the ram directions, direction of the Sun moving through the local interstellar cloud, and ani-ram directions. 


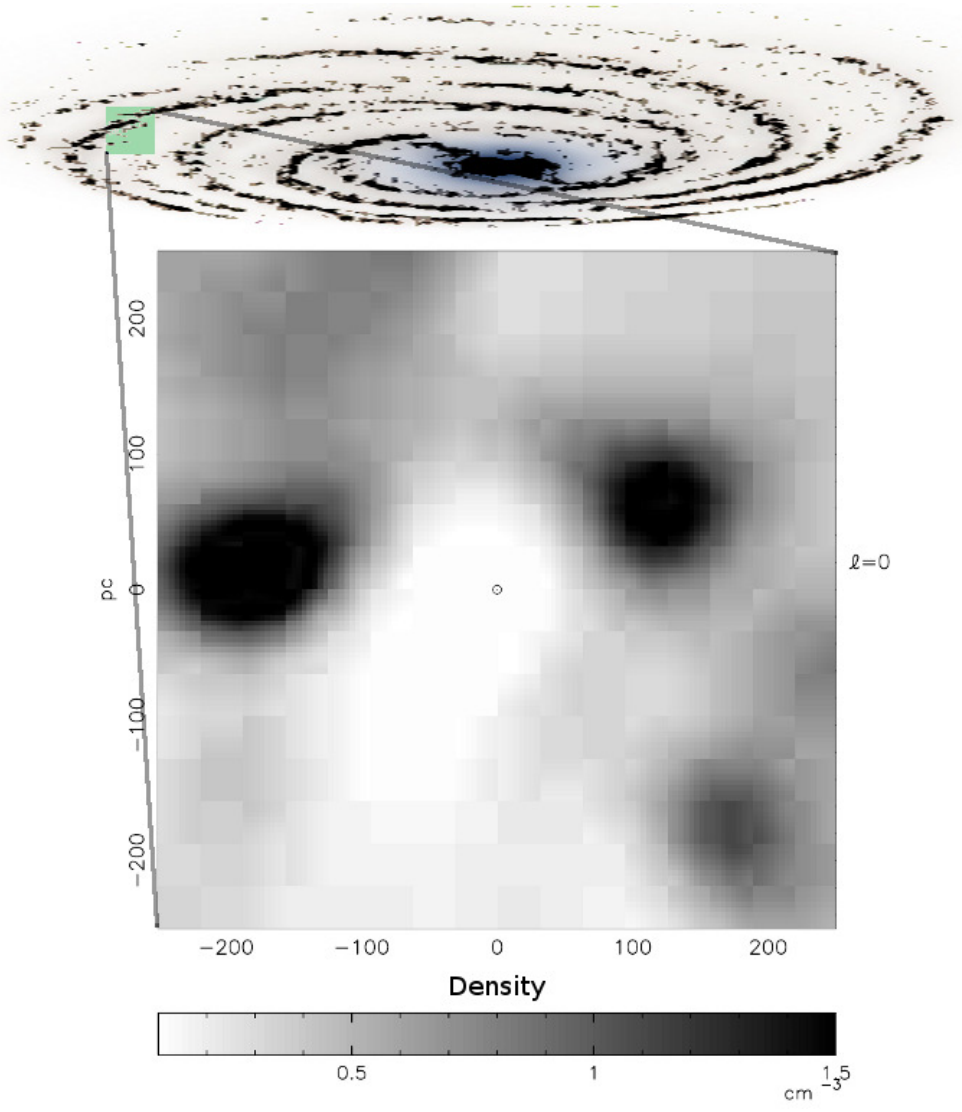

Fig. 2.39: Neutral atomic density mapping taken in the galactic plane up to 250 parsecs in all directions [79]. The large, low density region surrounding the origin is the local bubble. 
which may undergo CX collisions with interstellar neutral gases becoming nascent ENAs [80,81]. The second source is the LB-LIC interface as the LB contains very energetic, 110 $\mathrm{eV} / \mathrm{amu}$, ions which may penetrate into the LIC and undergo CX collisions, producing ENAs [82]. Utilizing the Monte Carlo (MC) transport, detailed in Sec. 2.2, the two ENA sources were investigated independently to determine their individual contribution to the local ENA background. No ions with solar origin are considered in this work as we are only interested in the effect of extra-solar (cosmic) sources on the local ENA background.

Determination of the local ENA background requires accurate cross sections for realistic transport simulations using MC methods. Elastic cross sections and CX cross sections, described in Sec. 2.1, were used to determine production of ENAs through CX collisions with neutral gases, ionization of ENAs through CX collisions with locally thermal ions, and energy-momentum transfer of ENAs through elastic collisions with locally thermal neutral gases. Figs. 2.40 and 2.41 show the mean free paths, Eq. 2.55, for hydrogen and helium ENAs in both the LIC and the LB environments. The total mean free path, combining all collision channels, is also shown in Figs. 2.40 and 2.41 to help demonstrate the contribution from the different collision channels. While being transported in the LIC, hydrogen ENAs mostly collide elastically with thermal hydrogen and occasionally undergo $\mathrm{CX}$ collisions with thermal $\mathrm{H}^{+}$, becoming hot ions. Helium ENAs, on the other hand, have very long mean free paths for CX collisions when their energies are below $6 \mathrm{keV}$ and so have a small probability of ever becoming hot ions once their initial neutralization has occurred. While the parameter of mean free path 
is important, it does not alone describe energy relaxation and transport of energetic particles. Complete descriptions of energy relaxation and transport require knowledge of projectile energy losses for single collisions, utilized with large ensembles of test particles.

Sec. 2.2.1 details the special treatment that is required for transporting ions through an environment which contains both ions, and magnetic fields. The ion densities listed above, $[45,46,3]$, in addition with a global, static interstellar magnetic field with a magnitude of $B_{0}=2.7 \mathrm{nT}$ and with a galactic coordinate direction of $\vec{B}(l, b)=\left(38^{\circ}, 23^{\circ}\right)$ [83] were utilized for both directional transport and ion drag energy loss.

Transport of hydrogen and helium ENAs produced by a single, Sun like star was simulated to obtain thermalization parameters as well as to investigate the effect of the static interstellar magnetic field during ion transport. Fig. 2.42 gives spatial density and energy probability distributions for hydrogen and helium ENAs as well as probability distributions for the test particles in energy-time. The ENA density probability distributions in Fig. 2.42 shows anisotropy for hydrogen ENAs as they will undergo CX collisions and have to be transported as ions, directed along the magnetic field. This effect is not seen for helium ENAs as the probability for CX collisions is very low during transport. The high probability for CX collisions by hydrogen ENAs also drastically shortens their thermalization length and times. The thermalzation lengths of the helium ENAs exceed that of the hydrogen ENAs by x100 while the thermalization times were roughly 10x longer for helium ENAs as compared with hydrogen ENAs. The drastic differences in the thermalization and transport properties of hydrogen ENAs as compared 

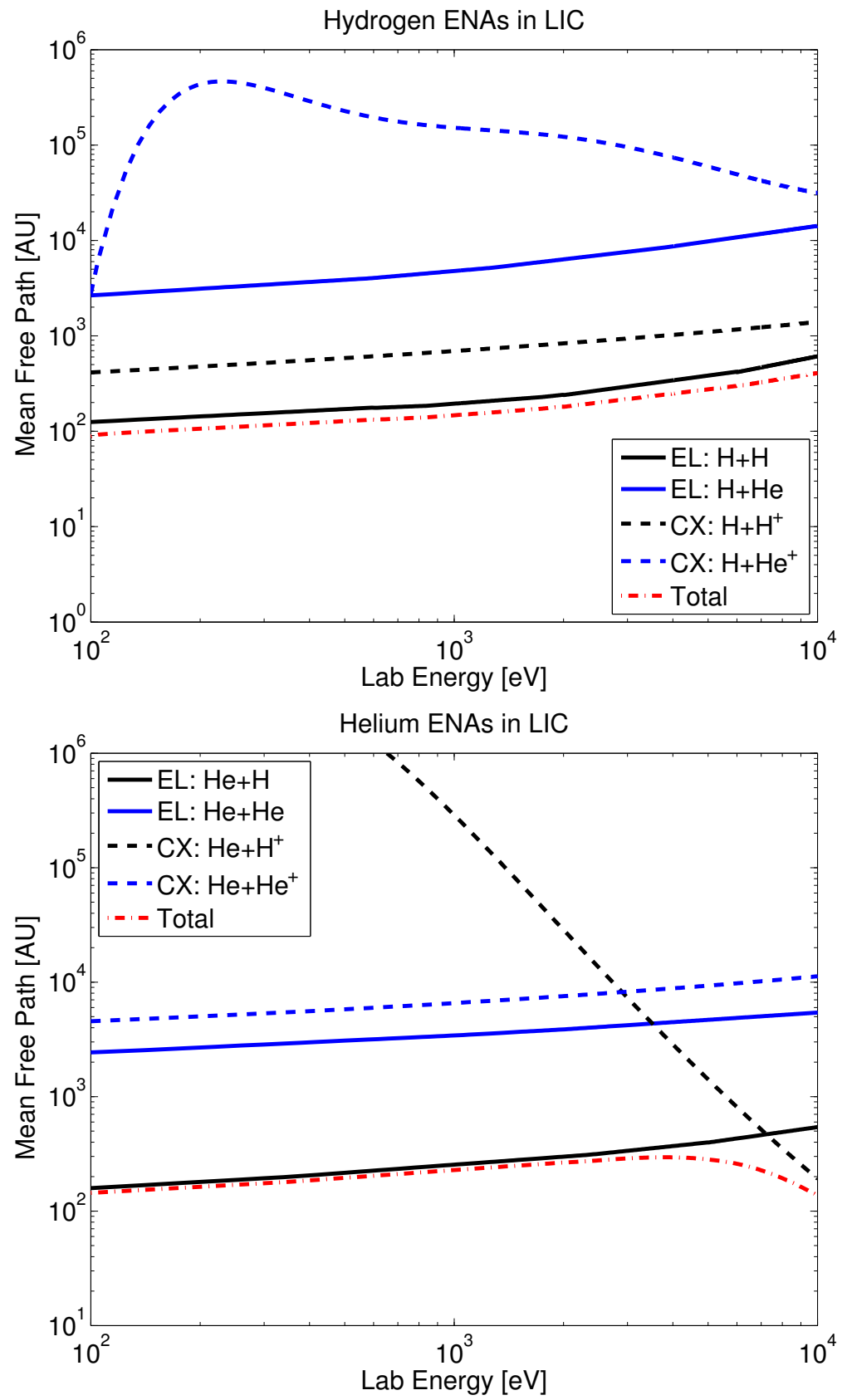

Fig. 2.40: Mean free path for hydrogen and helium ENAs begin transported through the LIC. Both elastic, atom-atom, and CX, atom-ion, collisions are shown utilizing densities of $\mathrm{H}=0.194 \mathrm{~cm}^{-3}, \mathrm{He}=0.015 \mathrm{~cm}^{-3}, \mathrm{H}^{+}=0.056 \mathrm{~cm}^{-3}$, and $\mathrm{He}^{+}=0.0096 \mathrm{~cm}^{-3}[45,46,3]$. Total cross sections, including all elastic and CX collisions channels, are shown as red dotted curves. 

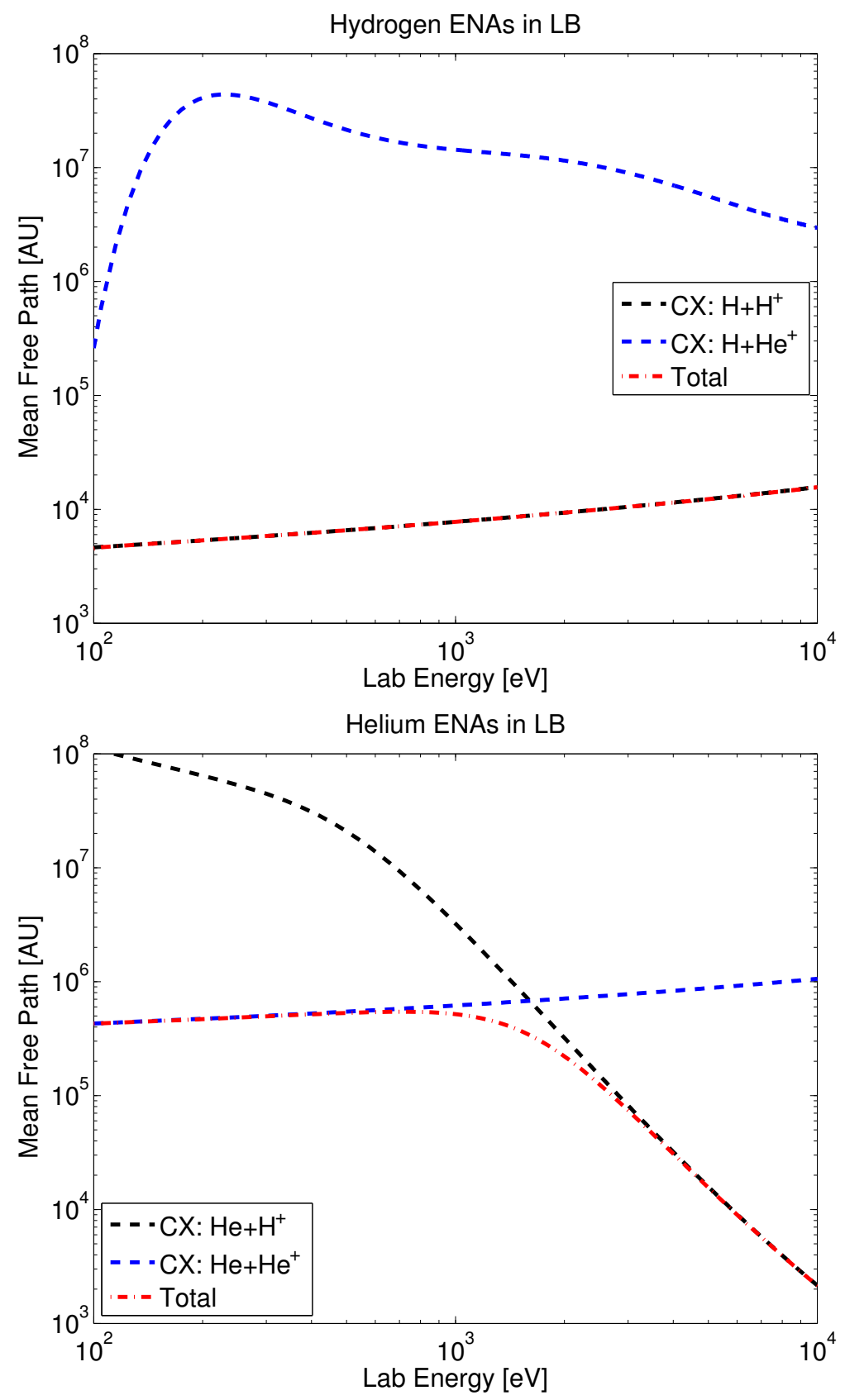

Fig. 2.41: Mean free path for hydrogen and helium ENAs begin transported through the LB. Atom-ion CX collisions are shown utilizing densities of $\mathrm{H}^{+}=0.005$ $\mathrm{cm}^{-3}$, and $\mathrm{He}^{+}=0.0001 \mathrm{~cm}^{-3}[45,46,3]$. Total cross sections, including all elastic and CX collisions channels, are shown as red dotted curves. 

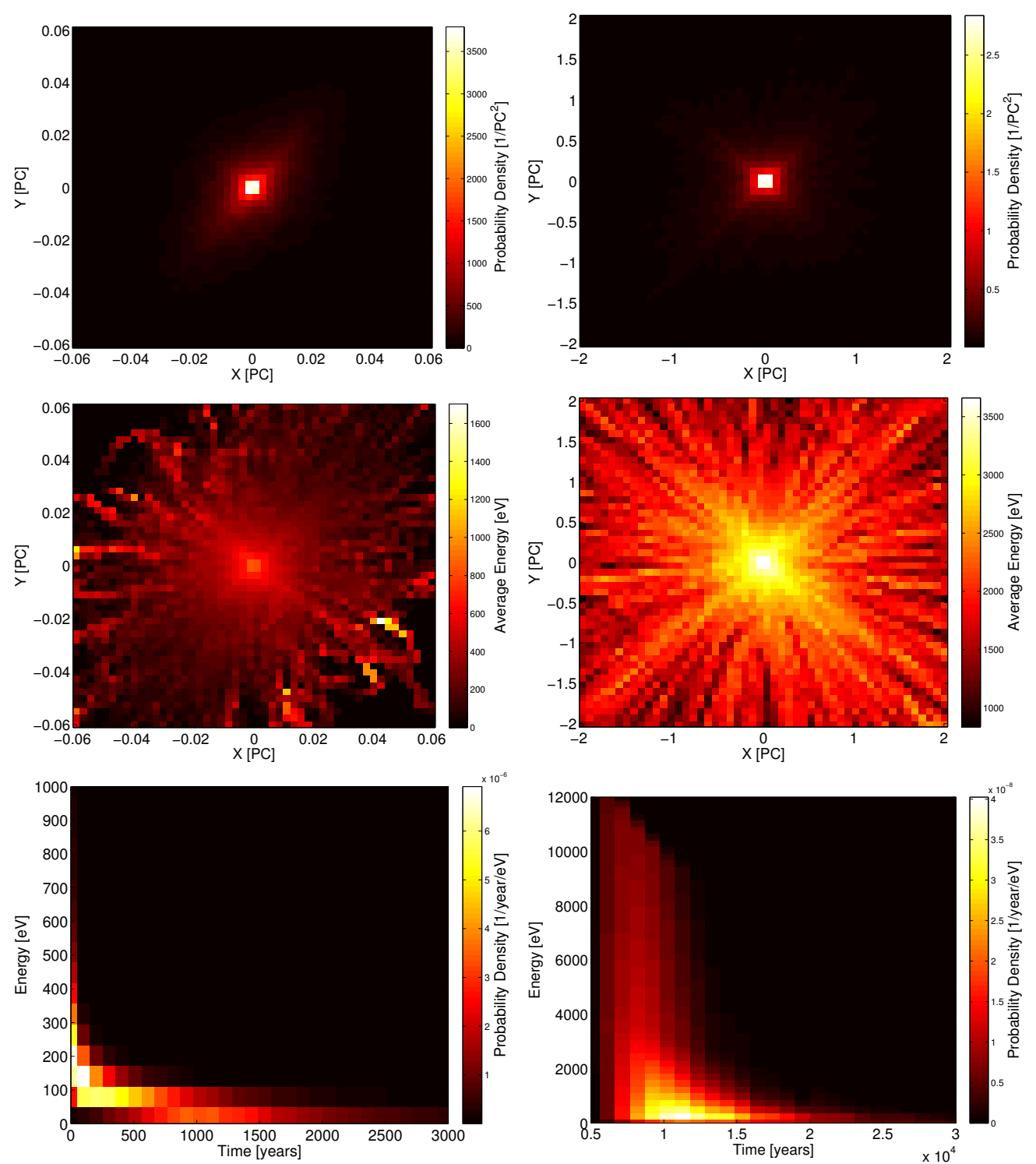

Fig. 2.42: Transport of hydrogen (left column) and helium (right column) ENAs from a single, Sun like star. The top figures shows the 2D spatial probability density for location of the ENAs while the middle figures show the 2D spatial average energy of the ENAs. The bottom figure gives a 2D probability for ENAs in energy-time coordinates to give an idea of the thermalization process. 


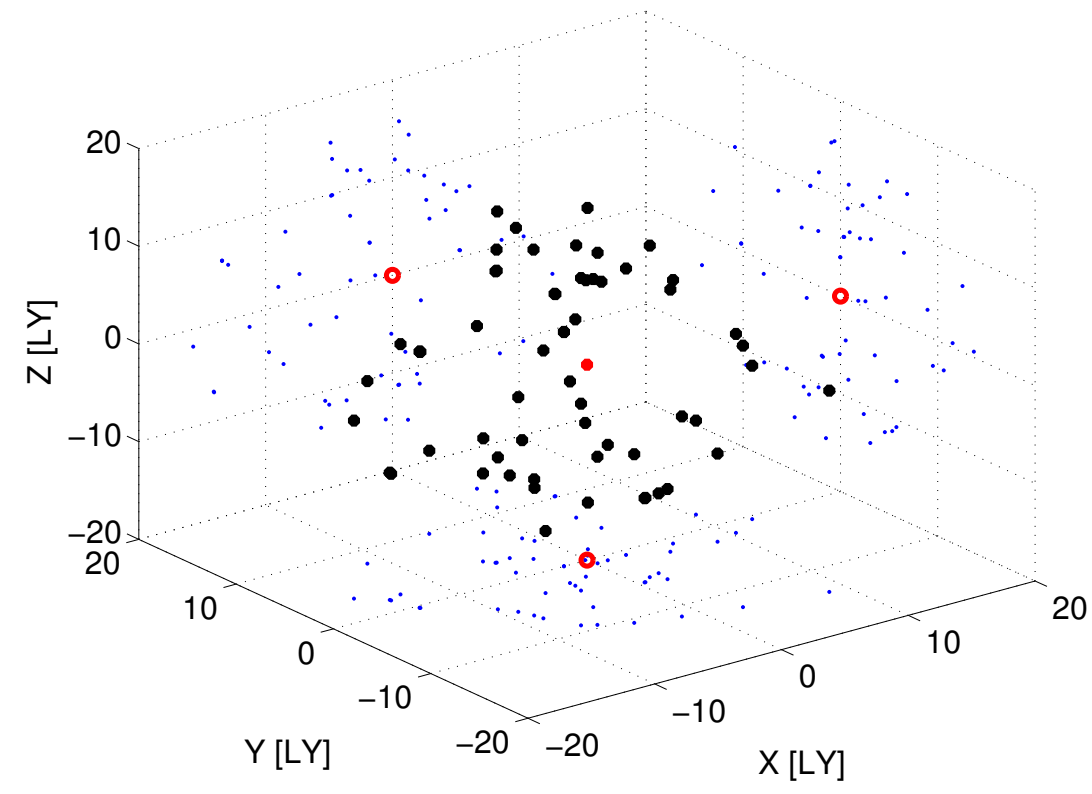

Fig. 2.43: $3 \mathrm{D}$ map of the 60 stars closes to the Sun. The Sun is shown as a red circle at the origin and projections of the nearest stars onto the $\mathrm{X}, \mathrm{Y}$, and $\mathrm{Z}$ planes is shown to help visualize the $3 \mathrm{D}$ distribution.

with the helium ENAs led to the conclusion that if an extra-solar background of ENAs exists within our solar system, helium would be the main constituent. For the remainder of this work, helium ENAs are considered exclusively for their long thermalization lifetimes transport lengths.

Within 17 LYs of our Sun resides 60 stars [84], each of which emit fast winds of ions [85]. Fig. 2.43 shows a 3D map of the 60 nearest stars with projections onto the $\mathrm{X}, \mathrm{Y}$, and $\mathrm{Z}$ planes and the Sun shown as a red circle for reference. To determine the background helium ENA flux due to the 60 nearest stars within the LIC, all stars were modeled to have the same stellar wind properties as the Sun. Additionally, the emission of stellar wind was modeled to be isotropic and stellar magnetic fields were 
neglected. 50,000 test particles were transported, distributed evenly among the 60 stars, and properties associated with thermalization and transport were recorded as a function of transport step, identical to the methods utilized for ENA transport in Mars, Sec. 2.3.

Steady state ENA distributions for density and energy were obtained within the LIC due to the stellar wind of the nearest 60 stars. Steady state distributions were obtained through recording of $2 \mathrm{D}$ slices of the $3 \mathrm{D}$ distributions at set intervals of transport steps. Defining the $i^{\text {th }}$ transport step 3D density distributions as $\rho_{(x, y, z)}^{i}$, the steady state distribution can be defined as

$$
\phi_{(x, y, z)}^{j}=\sum_{i=0}^{j} \rho_{(x, y, z)}^{i} \quad \mid \quad \phi_{(x, y, z)}^{j-1}=\phi_{(x, y, z)}^{j}=\phi_{(x, y, z)}^{j+1}=\phi_{(x, y, z)}^{j \rightarrow \infty}
$$

where the step $j$ is sufficiently large so that the system comes into a steady state. The steady state regime requires equilibrium in any part of the system phase space, whether that be spatial coordinates, momentum, or energy, as long as the flux of particles into a small component of phase space equals the flux leaving that same piece of phase space. The number of particles released during each transport step is determined utilizing average velocities and mean free paths to determine how much time has passed in the lab frame during the transport step, $\Delta t$. This method is effective in this simulation since the thermal gas has a uniform density, thus ensuring very uniform mean free paths for the ensemble. Fig. 2.44 displays steady states fluxes of helium ENAs in the LIC using $2 \mathrm{D}$ slices of the $3 \mathrm{D}$ distribution at $X=0, Y=0$, and $Z=0$ with the Sun at the origin. The flux was mapped in Fig. 2.44 through knowledge of steady state ENA densities, $n(x, y, z)$, as well as average energy distributions, $\bar{E}(x, y, z)$, so that the flux can be 
defined as

$$
\Gamma(x, y, z)=I_{0} \Delta t n(x, y, z) \bar{v}(x, y, z)
$$

where the average velocity $\bar{v}$ is found simply through the average kinetic energy $\bar{v}=$ $\sqrt{2 \bar{E} / m}$, and $I_{0}$ is the ion source intensity in units of [ions/sec]. For nearby stars, the ion source intensity was set to that of our Sun, $I_{0}=1.24 \times 10^{34} \mathrm{He}^{+} / \mathrm{s}$ and multiplied by 60 to account for all 60 stars.

Fig. 2.44 is displayed in $\log _{10}$ scaling of the flux to display the drastic changes in hot helium fluxes as a function of position within the LIC. Areas close to nearby stars in Fig. 2.44 may have fluxes several orders of magnitude higher than regions distant from any stars, as simple flux conservation dictates that the flux must fall off as $r^{-2}$ from point sources. The average flux of the three $2 \mathrm{D}$ slices in Fig. 2.44 is $4.8 \times 10^{-5} \mathrm{~cm}^{-2}$ $\mathrm{s}^{-1}$ and the average energy of the ENAs in this region is $1.5 \mathrm{keV}$. The detector area of IBEX for an ENA with an energy of $1.5 \mathrm{keV}$ is $0.12 \mathrm{~cm}^{2}$ [86], resulting in only $7.3 \times 10^{-7}$ ENAs/s, or 1 ENA per 2.3 years of observation. Theses extremely small fluxes make the ENA background due to nearby stars unobservable with the IBEX instrument, but could be observed with improvement of the neutral particle detectors in future satellite missions.

ENAs which originated at hot LB ions which penetrated into the LIC were also investigated in this work. Initial energy distributions of the ENAs were taken from the Maxwell-Boltzmann energy distributions for a temperature of $10^{6} \mathrm{~K}$ and with isotropic velocity distributions. The ENAs were then transported in an identical manner to those ENAs originating from nearby stars until they thermalized. Steady state distributions 

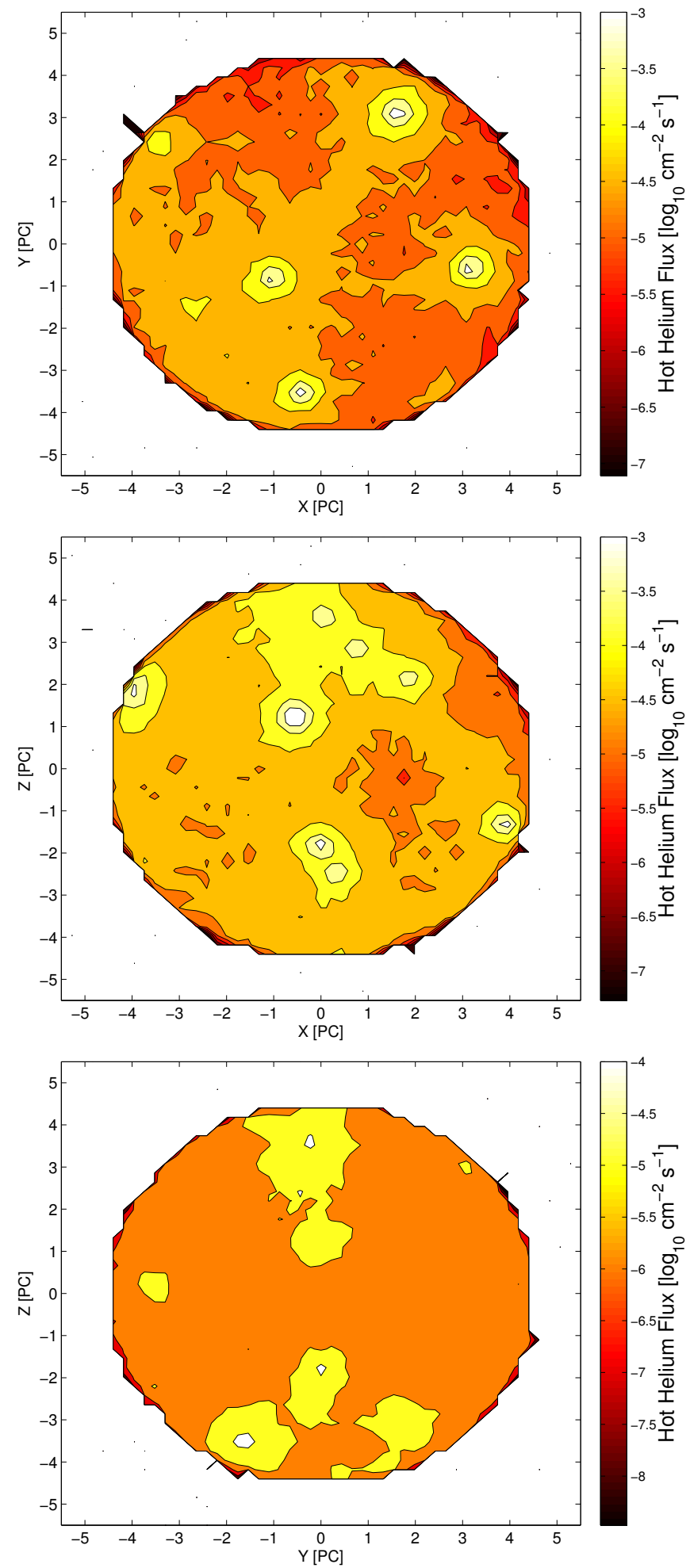

Fig. 2.44: Steady state helium ENA fluxes originating from nearby stars. $2 \mathrm{D}$ slices of the $3 \mathrm{D}$ distribution are shown for $X=0, Y=0$, and $Z=0$ with the Sun at the origin. 

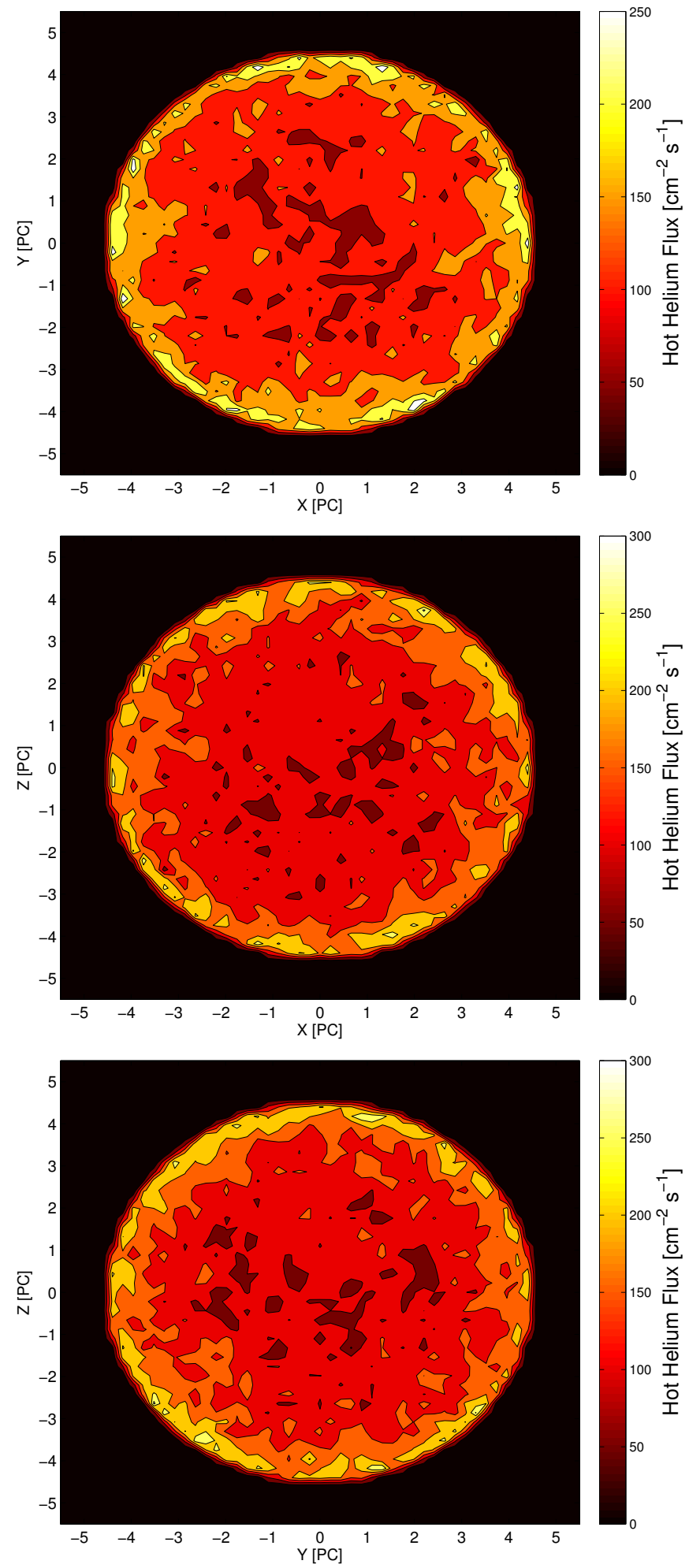

Fig. 2.45: Steady state helium ENA fluxes originating from the boundary between the hot LB and the LIC. 2D slices of the 3D distribution are shown for $X=0$, $Y=0$, and $Z=0$ with the Sun at the origin. 
for ENA density and energy were again obtained and utilized to calculated steady state flux maps as seen in Fig. 2.45. The fluxes were relatively uniform for Fig. 2.45 and are shown using linear scaling. Calculation of the total number of ions from the LB which may penetrate into the LIC was determined using simple estimations. Assuming isotropic velocity distributions of hot ions in the LB, the flux into the LIC was calculated to be $730 \mathrm{He}^{+} / \mathrm{cm}^{2} / \mathrm{s}$ using a $\mathrm{He}^{+}$density of $\mathrm{n}\left(\mathrm{H}^{+}\right)=0.0001 \mathrm{~cm}^{-3}$, and an average velocity of $\bar{v}=7.3 \times 10^{6} \mathrm{~cm} / \mathrm{s}$. The intensity was found my multiplying the flux by the surface area of the LIC, which was modeled to be spherical with a radius of $4.5 \mathrm{pc}[3,46]$. This calculation resulted in a total He ion intensity of $1.8 \times 10^{42} \mathrm{He} / \mathrm{c}$ which is roughly equivalent to $10^{8}$ stars. The average flux of the three $2 \mathrm{D}$ slices in Fig. 2.45 is $113 \mathrm{~cm}^{-2} \mathrm{~s}^{-1}$ and the average energy of the ENAs in this region is $83 \mathrm{eV}$. The detector area of IBEX for an ENA with an energy of $1.5 \mathrm{keV}$ is $0.015 \mathrm{~cm}^{2}$ [86], resulting in 13.5 ENAs/s observed by IBEX. Comparing this result to the background seen in Fig. 2.38 results in roughly $10 \%$ of the total background ENAs seen by the low energy IBEX instrument originating from the LB-LIC boundary. While the models for the LB and LIC utilized may be over simplified, the large fluxes observed in the steady state ENA distributions appear to be observable by current generation space telescopes, making them very important for current and future modeling. Certainly, more accurate calculations of the ENA fluxes produced by the LB-LIC interface could require detailed information in the morphology of the interface region and its physical characteristics. Nevertheless, these details should not change dramatically the estimated value of the ENA background. 
Background helium ENA distributions in our solar system were simulated due to both the local stellar neighborhood, consisting of 60 stars, as well as the interface between the LB and the LIC. Results of the simulation predict that the fluxes due to nearby stars is to low to detect with current generation instruments, but fluxes due to the LB-LIC boundary may constitute around $10 \%$ of the background ENAs seen be the IBEX low energy instrument. 


\section{Chapter 3}

\section{X-ray Scattering by Nanoparticles}

The observation of radiation from both ground and space based telescopes is the best way to study astrophysical objects and in the case of distant systems, radiation is the only method for obtaining information on the system. Emission and absorption spectra, transition line shifts, polarizations, and intensities are obtained with observational instruments and, combined with numerous statistical techniques, provide information on both the radiation emitters as well as the environments in which the radiation traverses to reach the detector.

$\mathrm{X}$-ray astronomy is particularly useful for observing distant, hot objects. With low absorption cross sections, keV X-rays may traverse through columns of gas on galactic scales, making them useful for observation of distant objects [87]. Traditionally, X-ray astronomy has focused on compact, high temperature systems such as neutron stars and black holes as these environments produce large fluxes of X-rays, outshining their visible spectrum by several orders of magnitude, and are thus easily observed [87]. Current generation X-ray telescopes have high angular resolution allowing for observation of both distant, point-like, X-ray emitters, as well as detection of X-rays halos around the

emitters. These X-ray halos are due to scattering of X-rays from nanoparticles consisting 
of both ice and dust, common in astrophysical environments [88-92]. Additionally, Xrays have been observed from cometary atmospheres due to both highly ionized solar wind charge exchanging with neutral gases, creating X-rays [93,94], as well as direct scattering and fluorescence of solar X-rays [95].

Nanoparticles are efficient scatterers of X-rays as their geometrical size is comparable to the wavelength of X-rays. Detailed knowledge of the scattering properties of X-rays by ice and dust nanoparticles, both being common in different astrophysical environments, gives greater insight into observed X-ray spectra from several objects ranging from planetary and cometary atmospheres to heliospheric dust clouds. In this work, simplified models have been built to describe the scattering of X-rays from nanoparti-

cles. The calculated scattering parameters were used to model solar X-rays interacting with both heliospheric dust and cometary atmospheres. Results obtained from these models were compared to available in situ data.

\subsection{Radiation Scattering Cross Sections}

Radiation scattering has been studied extensively for centuries, from Newton placing a prism in the path of a light beam [96] to Feynman describing quantum electrodynamics using probabilistic "Feynman Diagrams" [97]. Several different methods, approximations, and algorithms have been developed over the years to investigate radiation scattering for different atomic and molecular systems as well as bulk materials. The radiation-scatterer systems of interest in this work are X-rays incident on nanoparticles consisting of both dust and ice grains, common in many astrophysical environments 
[98-100]. Descriptions of the scattering system, calculation methods, and resulting cross sections follow.

To date, the majority of astrophysical observations that have been carried out were for micron-size grains as these are efficient scatterers of infrared and optical radiation which are easily seen by ground-based and satellite observations $[88,101]$. Nanoparticles are not efficient scatterers of infrared and optical radiation as the wavelength of these radiation domains is much larger than the geometrical size of the nanoparticles. However, nanoparticles should be more efficient in scattering X-ray radiation as their typical size $a$ is comparable with the wavelengths of X-ray photons, $a \sim \lambda$.

To model the scattering of X-rays by nanoparticles, the Mie theory was utilized exclusively for this work. The Mie theory gives the exact solution for a plane electromagnetic wave scattering elastically from a homogeneous dielectric sphere [102]. In reality, nanoparticles may take on much more complicated structures such as aggregate clusters or inhomogeneous core-mantle particles in which a core acts as a seed to which a mantle of different material attaches [98]. While more advanced models may be used to simulate these complicated dust structures, knowledge of scattering parameters for spherical, homogeneous nanoparticles gives a good approximation for modeling X-ray scattering from nanoparticles.

Using the Mie formalism [102], the differential cross section for the scattering of unpolarized radiation can be written as

$$
|f(\lambda, \theta)|^{2}=\frac{\lambda^{2}}{8 \pi^{2}}\left(\left|S_{1}(\theta)\right|^{2}+\left|S_{2}(\theta)\right|^{2}\right)
$$

where $\lambda$ is the wavelength of the radiation and $\theta$ is the scattering angle. The complex 
scattering amplitudes $S_{1}(\theta)$ and $S_{2}(\theta)$ are defined as

$$
\begin{aligned}
& S_{1}(\theta)=\sum_{n=1}^{\infty} \frac{2 n+1}{n(n+1)}\left[a_{n} \pi_{n}(\cos \theta)+b_{n} \tau_{n}(\cos \theta)\right] \\
& S_{2}(\theta)=\sum_{n=1}^{\infty} \frac{2 n+1}{n(n+1)}\left[b_{n} \pi_{n}(\cos \theta)+a_{n} \tau_{n}(\cos \theta)\right]
\end{aligned}
$$

where the angular functions $\pi_{n}(\cos \theta)$ and $\tau_{n}(\cos \theta)$ are defined as

$$
\pi_{n}(\cos \theta)=\frac{1}{\sin \theta} P_{n}^{1}(\cos \theta) \quad \text { and } \quad \tau_{n}(\cos \theta)=\frac{d}{d \theta} P_{n}^{1}(\cos \theta)
$$

with $P_{n}^{m}(\cos \theta)$ being the associated Legendre polynomials. The amplitude functions $a_{n}$ and $b_{n}$ in Eq. 3.2 are defined using the Riccati-Bessel functions $\psi_{n}(z)$ and $\chi_{n}(z)$ which are closely related to spherical Bessel functions, $j_{n}(z)$ and $n_{n}(z)$

$$
\begin{array}{r}
\psi_{n}(z)=z j_{n}(z), \\
\chi_{n}(z)=-z n_{n}(z), \\
\zeta_{n}(z)=\psi_{n}(z)+i \chi_{n}(z) .
\end{array}
$$

Defining the parameters

$$
x=k a=\frac{2 \pi a}{\lambda} \quad \text { and } \quad y=m k a
$$

with $k$ and $m$ being the wavenumber of the radiation and complex refractive index of the sphere respectively, in addition to Eqs. 3.3 and 3.4, the amplitude functions $a_{n}$ and $b_{n}$ are defined as

$$
\begin{aligned}
a_{n} & =\frac{\psi_{n}^{\prime}(y) \psi_{n}(x)-m \psi_{n}(y) \psi_{n}^{\prime}(x)}{\psi_{n}^{\prime}(y) \zeta_{n}(x)-m \psi_{n}(y) \zeta_{n}^{\prime}(x)}, \\
b_{n} & =\frac{m \psi_{n}^{\prime}(y) \psi_{n}(x)-\psi_{n}(y) \psi_{n}^{\prime}(x)}{m \psi_{n}^{\prime}(y) \zeta_{n}(x)-\psi_{n}(y) \zeta_{n}^{\prime}(x)} .
\end{aligned}
$$

Primed values in Eq. 3.6 refer to the respective function's derivatives such that

$$
\psi_{n}^{\prime}(z)=\frac{d}{d z} \psi_{n}(z) \quad \text { and } \quad \zeta_{n}^{\prime}(z)=\frac{d}{d z} \zeta_{n}(z)
$$




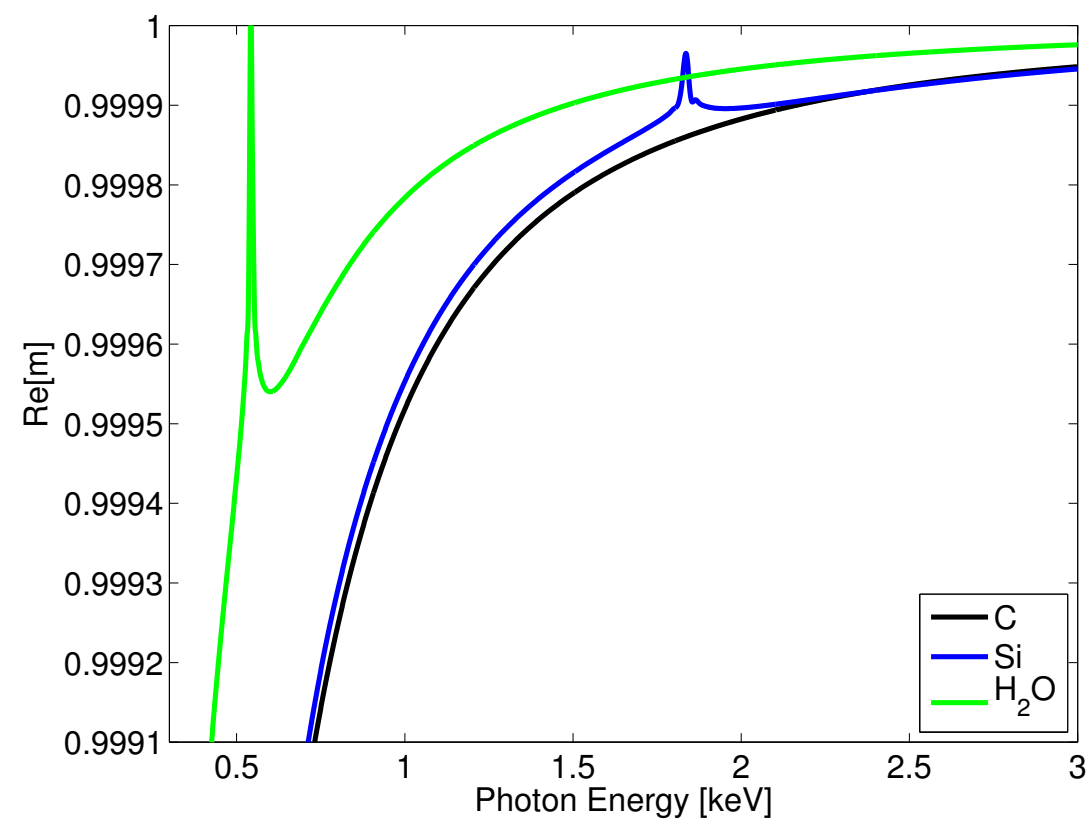

Fig. 3.1: Real components of complex indices of refraction

The implementation of the Mie theory was achieved using robust numerical algorithms $[103]$.

The composition of the nanoparticles considered consists of bulk silicon, carbon, and $\mathrm{H}_{2} \mathrm{O}$, which are common elements found in astrophysical nanoparticles [98]. Accurate, wavelength dependent, complex refractive indices were used in the Mie calculations for the different sphere compositions [104]. Figs. 3.1 and 3.2 display the real and imaginary parts of the indices of refraction for silicon, carbon, and ice nanoparticles as a function of incident radiation wavelength. Resonances can be seen in Figs. 3.1 and 3.2 for silicon and the oxygen from ice, resulting from the $\mathrm{K}$ edge at $1.84 \mathrm{keV}$ and $0.5 \mathrm{keV}$ respectively [105].

With knowledge of accurate, energy dependent indices of refraction for bulk sil- 


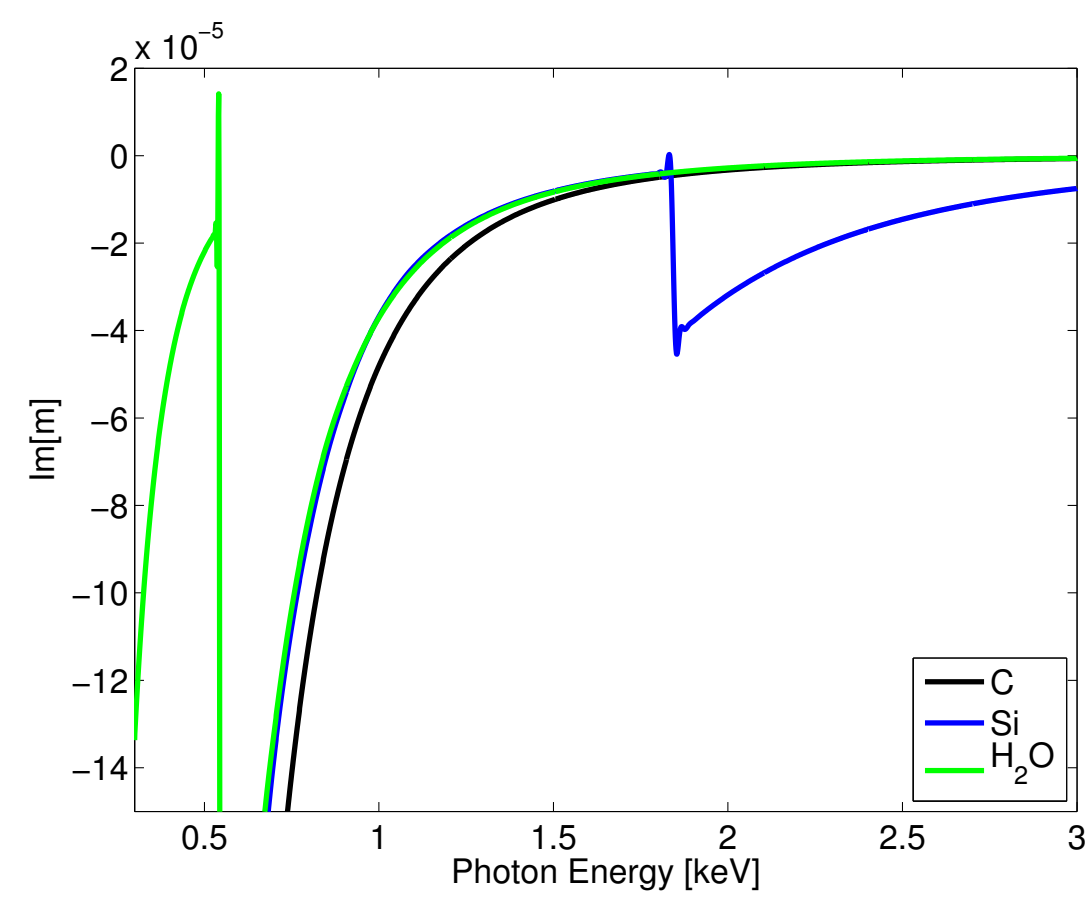

Fig. 3.2: Imaginary components of complex indices of refraction

icon, carbon, and ice particles, differential scattering cross sections can be computed utilizing Eq. 3.1. These calculated differential cross sections are shown in Figs. 3.3, 3.4, and 3.5 in 3D plots which shows the energy-angular dependent differential cross sections for photon energies ranging from 1 to $3 \mathrm{keV}$. The differential cross sections in Figs. 3.3 through 3.5 all shown similar behaviors with more complex structures and preferential scattering directions for larger nanoparticle grains. Additionally, the differential cross sections for silicon and ice grains shown in Figs. 3.4 and 3.5 display smaller differential cross sections for photons with energies near $1.8 \mathrm{keV}$ and $0.5 \mathrm{keV}$ respectively, where the $\mathrm{K}$ shells result in an absorption peaks, seen in Fig. 3.2. These differential cross sections are used extensively for heliospheric dust scattering and cometary atmosphere nanoparticle scattering where the emitter-scatterer-detector system is fixed and thus 

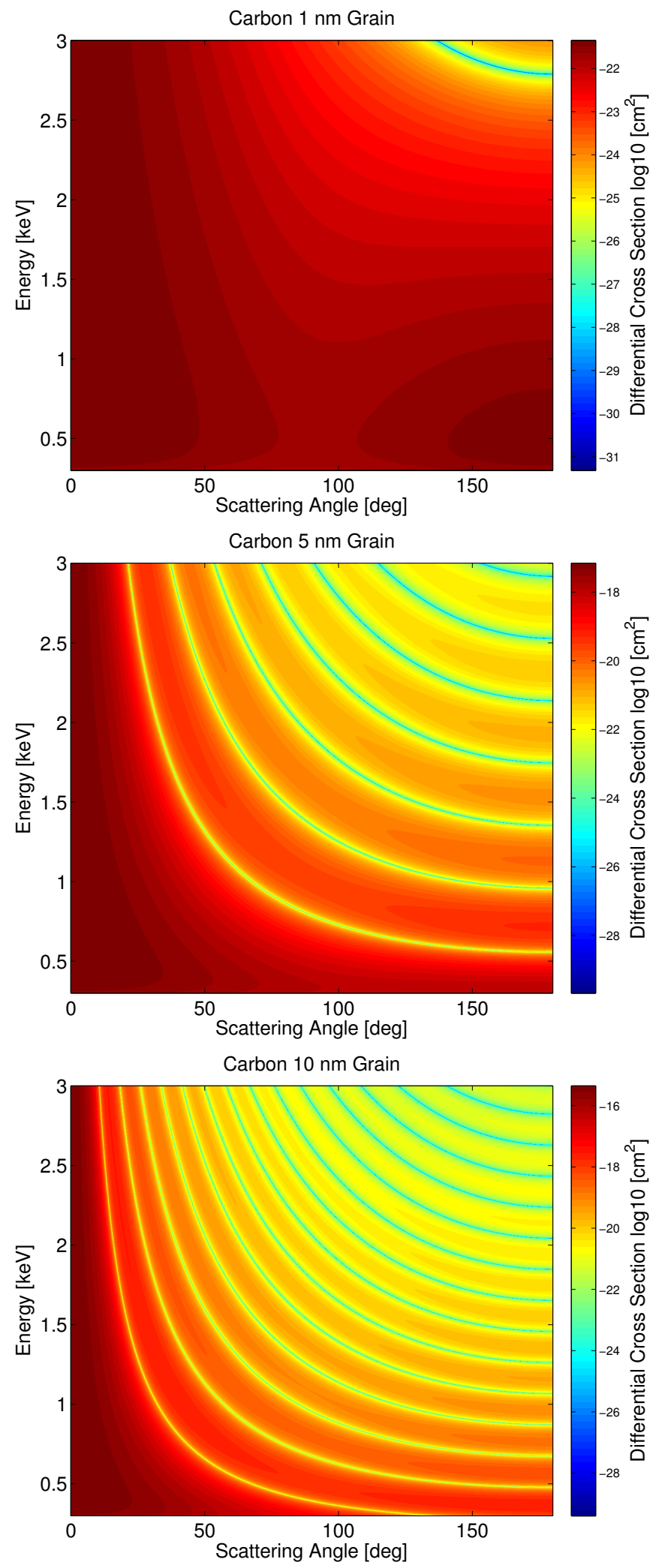

Fig. 3.3: Differential cross sections for $X$-ray scattering from carbon grains 

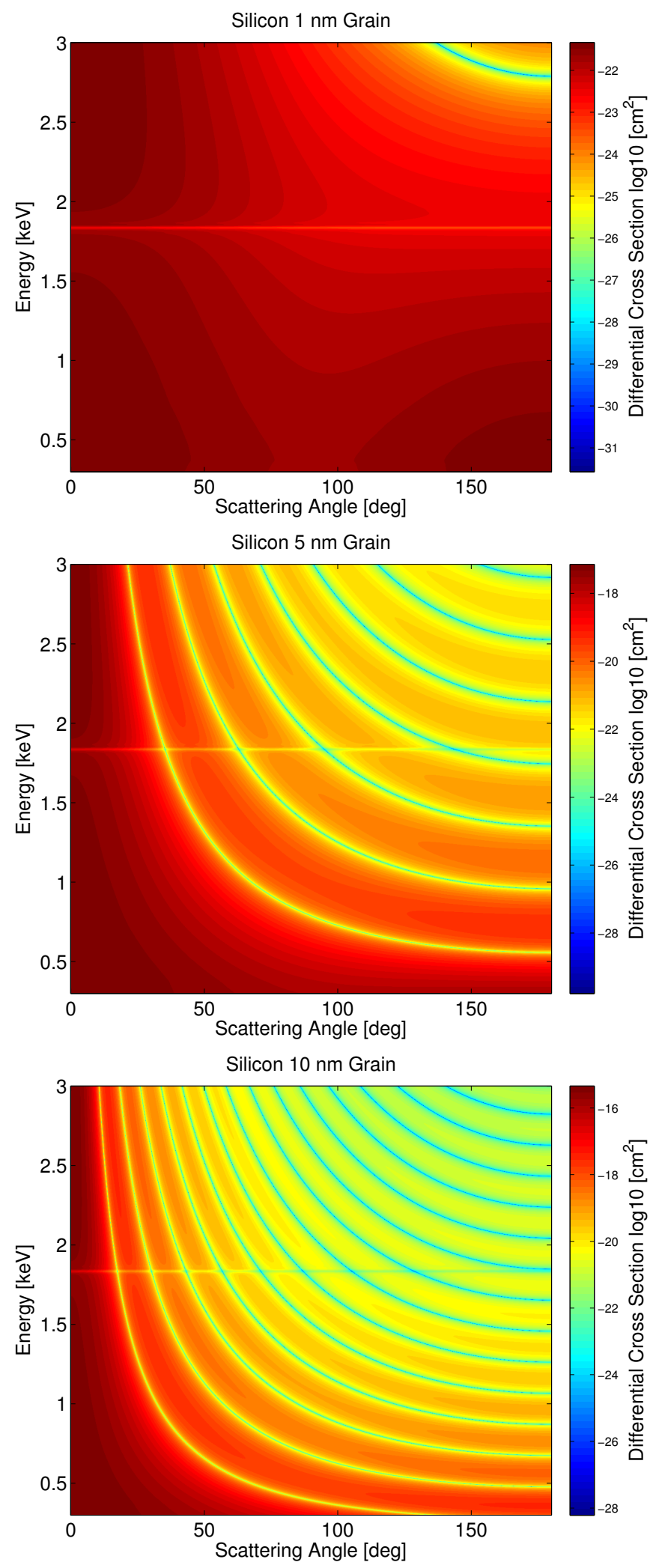

Fig. 3.4: Differential cross sections for X-ray scattering from silicon grains 

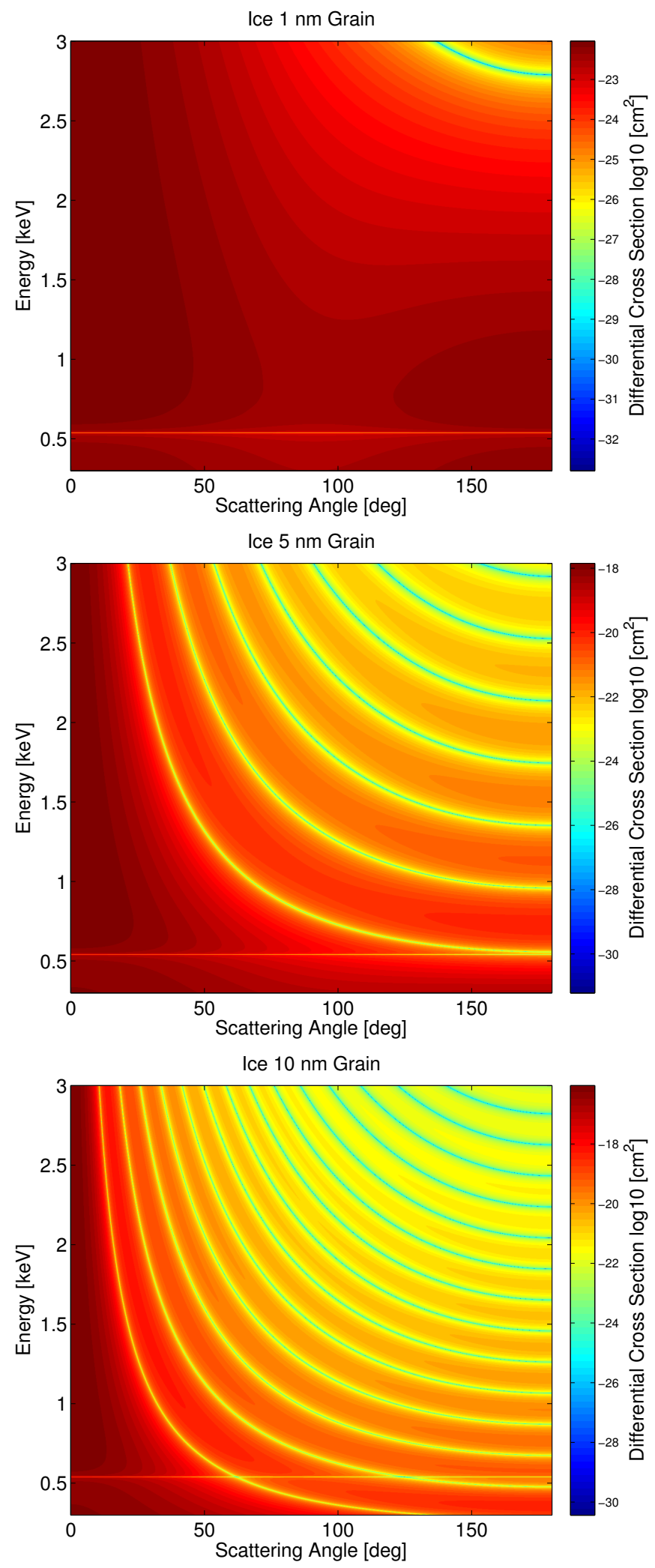

Fig. 3.5: Differential cross sections for X-ray scattering from ice grains. 


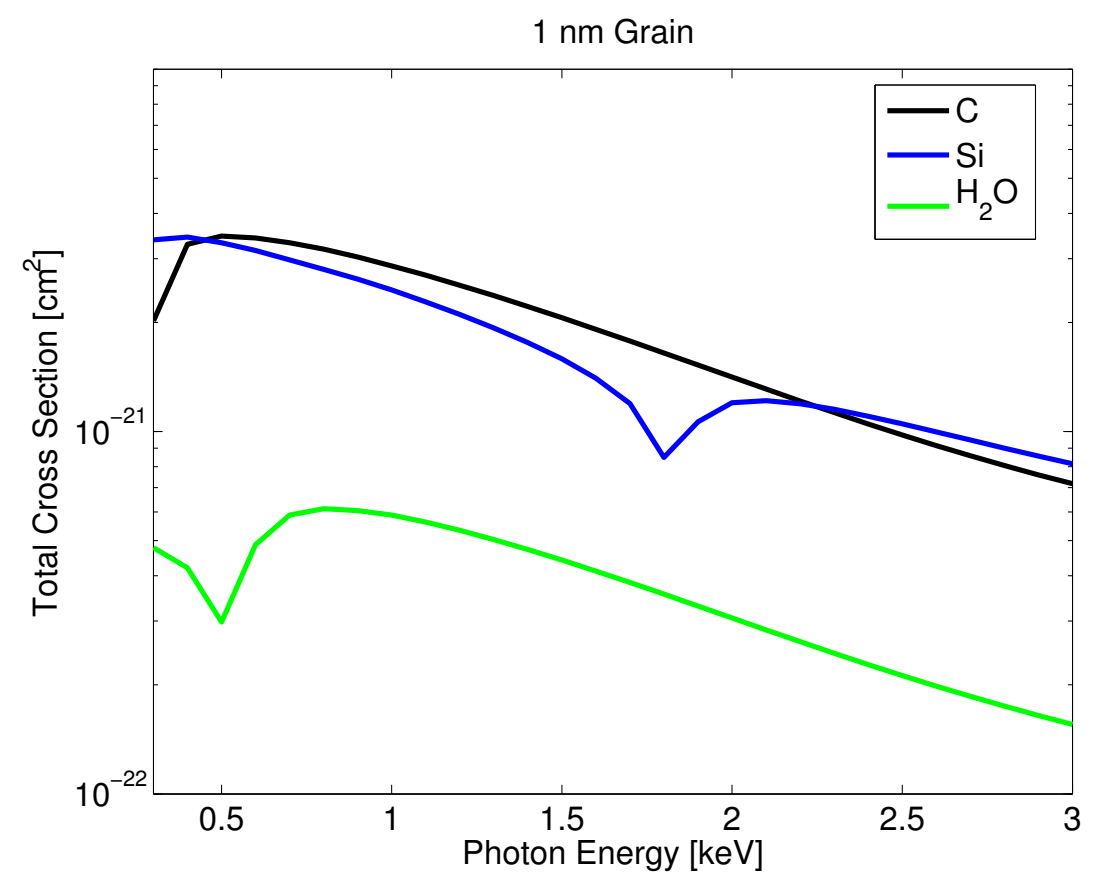

Fig. 3.6: Total cross sections for $X$-ray scattering from $1 \mathrm{~nm}$ grains

only a small range of scattering angle contribute to a particular observation.

Total scattering cross sections may also be calculated through integration of the differential cross sections, Eq. 3.1, over all scattering angles:

$$
\sigma(\lambda)=\int|f(\lambda, \theta)|^{2} d \Omega=2 \pi \int_{0}^{\pi} \sin \theta|f(\lambda, \theta)|^{2} d \theta .
$$

The total scattering cross sections were calculated for X-ray photons with energies ranging from 1-3 keV for 1, 5, and $10 \mathrm{~nm}$ grains consisting of carbon, silicon, and ice. Figs. 3.6, 3.7, and 3.8 display these total scattering cross sections with each figure displaying the total cross sections for all three grains types for a given grain size for comparison. Again, the K shell absorption at $1.8 \mathrm{keV}$ can be seen in the silicon total cross sections as the value decreases about this photon energy for all three grain sizes.

With knowledge of both the total and differential scattering cross sections for 


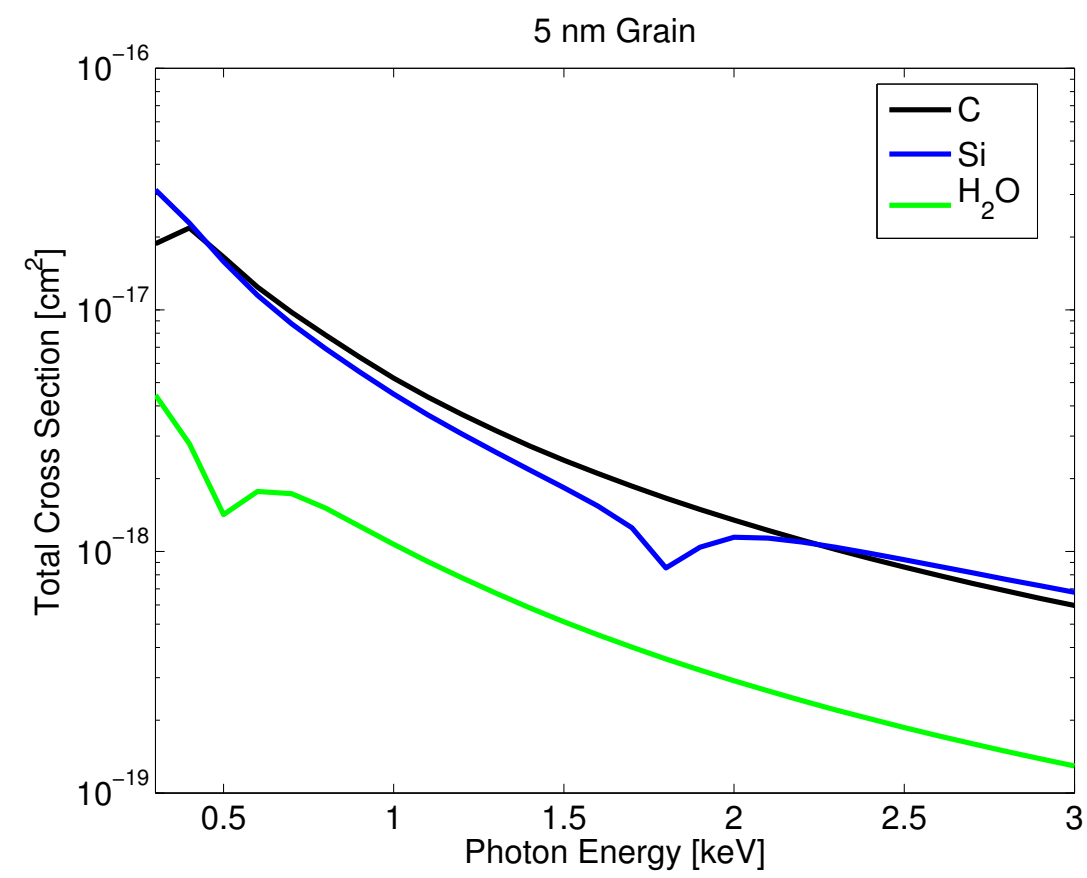

Fig. 3.7: Total cross sections for X-ray scattering from $5 \mathrm{~nm}$ grains

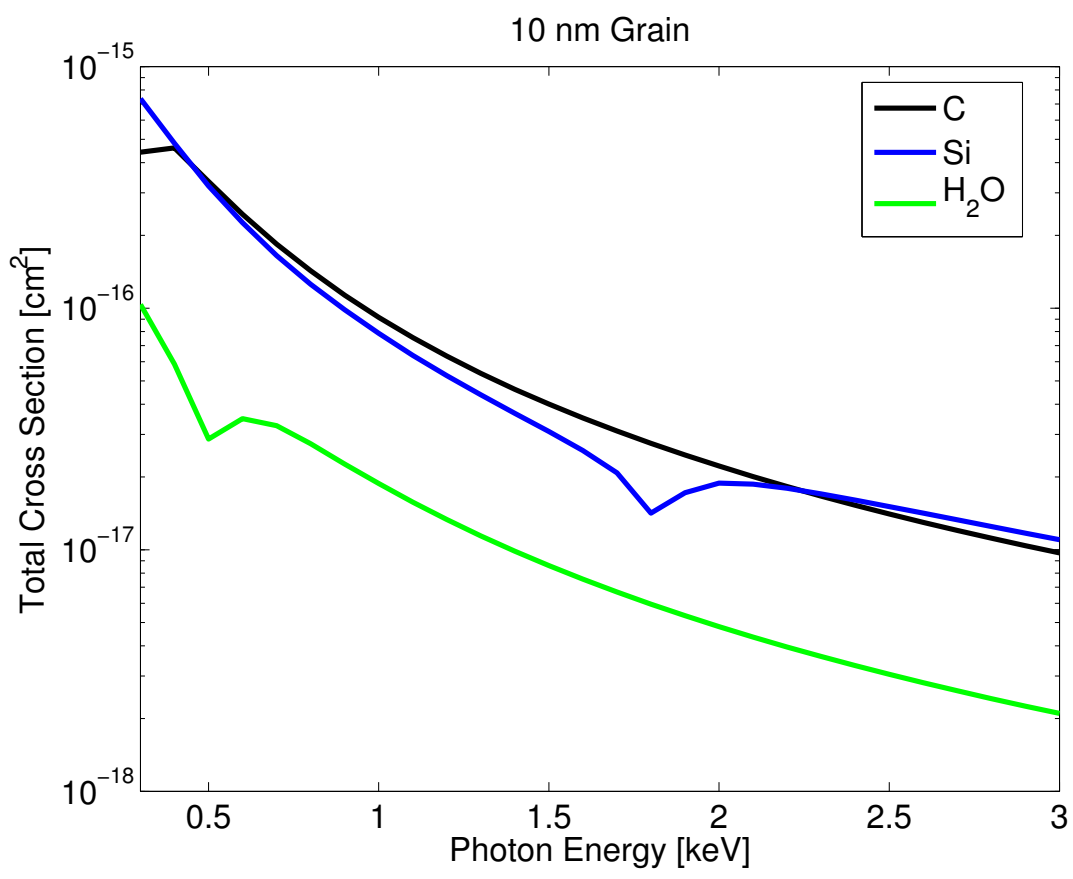

Fig. 3.8: Total cross sections for X-ray scattering from $10 \mathrm{~nm}$ grains 
nanoparticles consisting of bulk carbon, silicon, and ice, and with grain sizes ranging from 1 to $10 \mathrm{~nm}$ in radius, the scattering of X-rays in different astrophysical environments by nanoparticles may be investigated accurately. In the following sections these cross sections are put to use to model solar X-ray scattering by heliospheric dust clouds, and solar X-ray scattering by nanoparticles in cometary atmospheres.

\subsection{Heliosphere Dust Scattering}

In the recent years much attention has been paid to nanoparticles within the solar system $[106,99,107]$. While the origin and composition of these nanoparticles may differ drastically, a fundamental similarity in their interaction with radiation is expected based purely on the geometric size and shape of the nanoparticles. Within the heliospheric region inside $1 \mathrm{AU}$, parameters of velocity, charge, and size distributions have been analyzed and computed for nanoparticles consisting mainly of silicon and carbon dust [99]. In particular, it is thought that nanoparticles with a radius smaller than $10 \mathrm{~nm}$ are trapped within a region around the sun between 0.1 and $0.2 \mathrm{AU}$ [99]. It is the goal of this work to investigate the scattering of solar X-ray from these nanoparticle distributions and to determine if a scattered X-ray halo may be observed surrounding the sun.

To determine global scattering parameters for the nanoparticles surrounding the Sun, spatial and size distributions of the nanoparticles must be utilized. A simplified distribution was used for particles with radii less than $10 \mathrm{~nm}$ a distance $r$ from the Sun 
such that

$$
\frac{\partial n(r, a)}{\partial a} \simeq 2.5 n_{d} \frac{a_{\min }^{3.5}}{a^{3.5}}\left(\frac{R_{0}}{r}\right)^{2}
$$

where $a$ is the grain radii, $n_{d}=1.5 \times 10^{-10} \mathrm{~cm}^{-3}, a_{\min }=3 \mathrm{~nm}$, and $R_{0}=1 \mathrm{AU}$ [108]. The spatial nanoparticle distribution for all grain sizes is obtained from Eq. 3.9 as

$$
n(r)=n_{d}\left(\frac{R_{0}}{r}\right)^{2}
$$

Integrating Eq. 3.10 from $r_{\min }=0.1 \mathrm{AU}$ to $r_{\max }=1 \mathrm{AU}$ gives the total number of nanoparticles as $N_{t o t}=4.6 \times 10^{29}$ particles.

In addition to the realistic nanoparticle distributions from Eqs. 3.9 and 3.10, a uniform shell model was also utilized for comparison. The uniform shell distribution ranged from $r_{\text {min }}=0.08 \mathrm{AU}$ to $r_{\max }=0.1 \mathrm{AU}$ with a constant nanoparticle density such that the total number of particles was equal to that of the realistic nanoparticle distribution $N_{t o t}=4.6 \times 10^{29}$ particles [108].

With knowledge of scattering parameters for X-rays incident on nanoparticles consisting of silicon and carbon, Section 3.1, along with spatial and size distributions of nanoparticles, Eqs. 3.9 and 3.10, the total scattered X-ray intensity may be computed. Fig. 3.9 shows a scattering diagram for the Sun-nanoparticle-detector system. In Fig. $3.9, R_{0}$ is again the earth-Sun distance of $1 \mathrm{AU}, r_{d}$ is the nanoparticle-detector distance, $r_{g}$ is the Sun-nanoparticle distance, and $\theta_{s c}$ is the scattering angle. The ratio of scattered X-ray intensity, $I_{s}(\lambda)$, to the emitted solar X-ray intensity at $1 \mathrm{AU}, I_{R_{0}}(\lambda)$, may then be found through integration as

$$
\frac{I_{s}(\lambda)}{I_{R_{0}}(\lambda)}=\int_{r_{t}}^{R_{0}} \int_{0}^{\pi} \int_{0}^{2 \pi} d r \sin \theta d \theta d \phi \int_{a_{\min }}^{a_{\max }} d a \frac{\partial n(r, a)}{\partial a} \frac{\left|f\left(\lambda, \theta_{s c}, a\right)\right|^{2}}{1+\frac{r^{2}}{R_{0}^{2}}-2 \frac{r}{R_{0}} \cos \theta}
$$




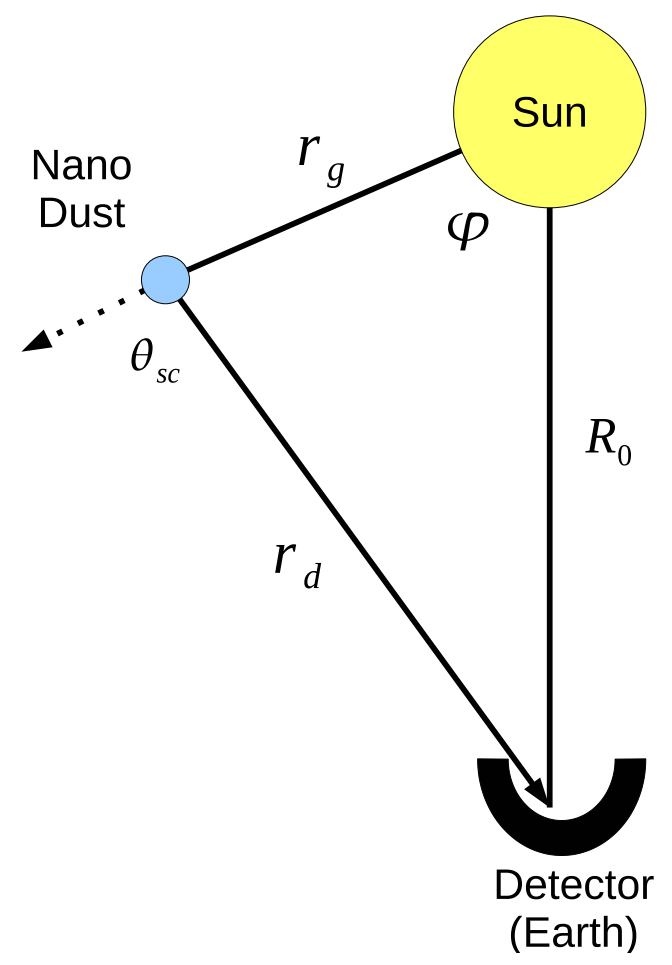

Fig. 3.9: Sun-nanoparticle-detector scattering diagram 


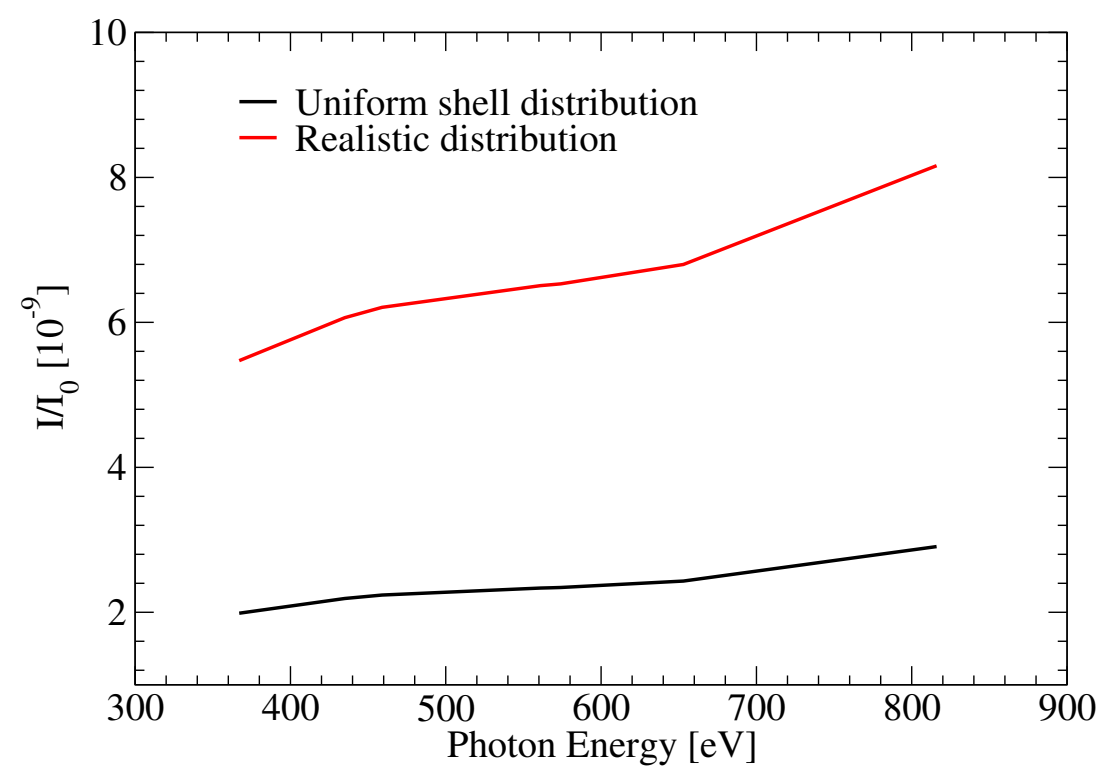

Fig. 3.10: Ratio of incident to scattered X-ray intensity due to nanoparticles in the heliosphere. Scattered intensity ratio shown for the realistic nanoparticle distribution as well as the uniform spherical shell distribution.

where the scattering angle is defined in terms of the polar angle as

$$
\theta_{s c}=\theta+\arctan \frac{r \sin \theta}{R_{0}-r \cos \theta}
$$

the minimum radial integration $r_{t}$ is $0.1 \mathrm{AU}$, and $\left|f\left(\lambda, \theta_{s c}, a\right)\right|^{2}$ is the differential scattering cross section [108].

Calculation of Eq. 3.11 was carried out for carbon nanoparticles over the energy interval from 350 to $850 \mathrm{eV}$ using both the realistic and uniform shell nanoparticle distributions. The ratio of scattered to incident intensity as a function of photon energy for both distributions is shown in Fig. 3.10 [108]. It is clear from Fig. 3.10 that the spatial distribution of nanoparticle scatterers plays an important role in overall scattering intensity as the realistic distribution, with the same number of scatterers as the uniform 
shell distribution, produces fluxes which are larger by a factor of 3 from the uniform shell distribution. The total intensity of X-ray photons, scattered by nanoparticles, can be evaluated for the known flux of the solar X-ray photons $I_{R_{0}}(\lambda)$. For the interval of photon energies between 290 and $530 \mathrm{eV}$ the photon flux is about $1.6 \times 10^{8} \mathrm{ph} / \mathrm{cm}^{2} / \mathrm{s}$ and $0.2 \times 10^{8} \mathrm{ph} / \mathrm{cm}^{2} / \mathrm{s}$ for photons with energies greater than $530 \mathrm{eV}$ at the solar minimum [100]. During a solar flare, the X-ray flux may increase by 1 to 2 orders of magnitude. The total line of sight X-ray intensity, integrated over the entire sky, may then be found over the energy interval from $350 \mathrm{eV}$ to $1 \mathrm{keV}$, estimated at solar minimum $I_{s c}^{\text {min }}$ and during a solar flare $I_{s c}^{f}$ using the results from Fig. 3.10 [108]:

$$
I_{s c}^{\min } \sim 8 \frac{\mathrm{ph}}{\mathrm{cm}^{2} \mathrm{~s}} \quad \text { and } \quad I_{s c}^{f} \sim 350 \frac{\mathrm{ph}}{\mathrm{cm}^{2} \mathrm{~s}} .
$$

During solar minimum conditions, the intensity of X-rays, scattered by nanoparticles, is slightly smaller than the diffuse X-ray background, but during strong X-ray flares, the nanoparticles could be seen indirectly from the scattered X-rays for the short duration of the flare. Scattered X-ray flares have already been detected from the Jupiter atmosphere [109], and with these new nanoparticle scattering calculations we predict that they could also be observed from the inner solar system.

\subsection{X-ray Scattering from Cometary Atmospheres}

The emission of X-rays from cometary atmospheres was first observed in 1996 using the Roentgensatellit (ROSAT) observatory to study comet Hyakutake [110]. These observations were quickly explained through charge exchange (CX) collisions between heavy, highly ionized solar wind (SW) and neutral gases in the cometary atmosphere [111]. 


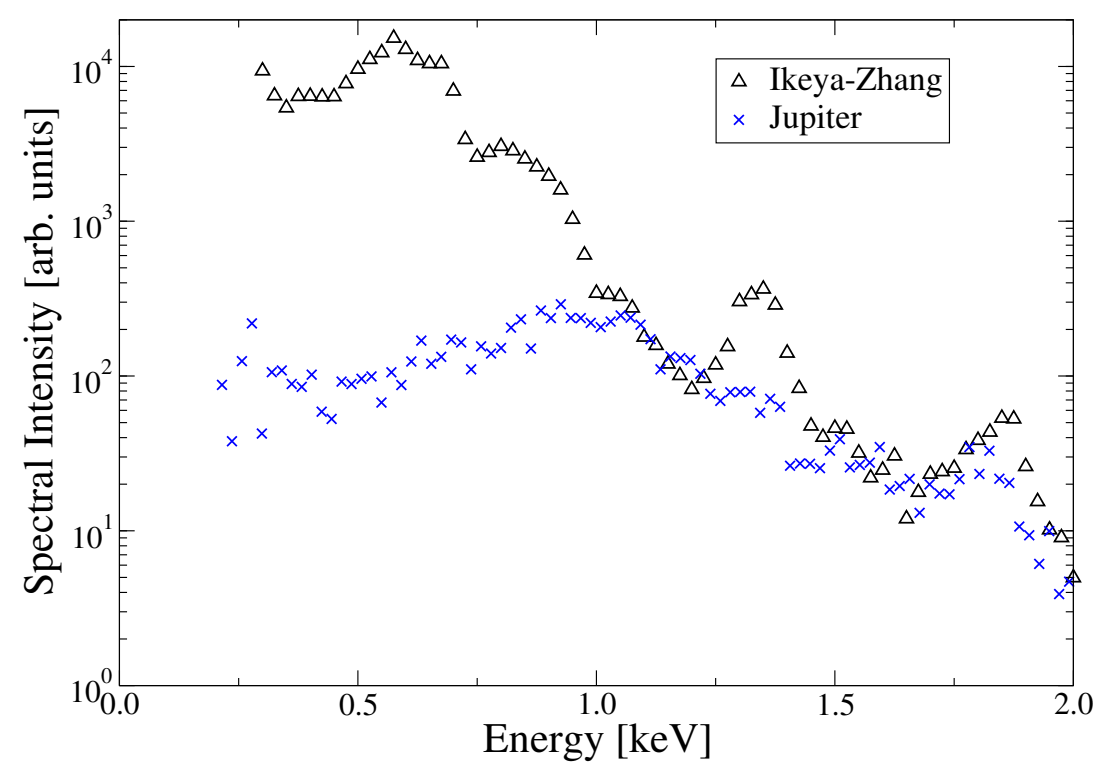

Fig. 3.11: Comparison of X-ray spectrum from comet Ikey-Zhang [93] and Jupiter [113], observed during solar X-ray flares. The data sets have been scaled to overlay together in order to better visualize the strong similarity in spectral structure between the two astronomical objects at photon energies above 1 $\mathrm{keV}$.

Highly ionized SW is an effective source for CX in cometary atmospheres producing Xrays with energies below $1 \mathrm{keV}$, but higher energy photons require very highly ionized $\mathrm{Mg}^{11+}$ and $\mathrm{Si}^{13+}$ or other exotic heavy ions which have not been observed in the $\mathrm{SW}$ $[93,112]$.

To further investigate the origin of these hard cometary X-rays, the spectrum of recently observed comet Ikey-Zhang [93] was compared with that of the Jovian atmosphere [113], both observations being conducted during solar flare conditions. Fig. 3.11 displays observational X-ray spectra from comet Ikey-Zhang and the Jupiter disk up to 


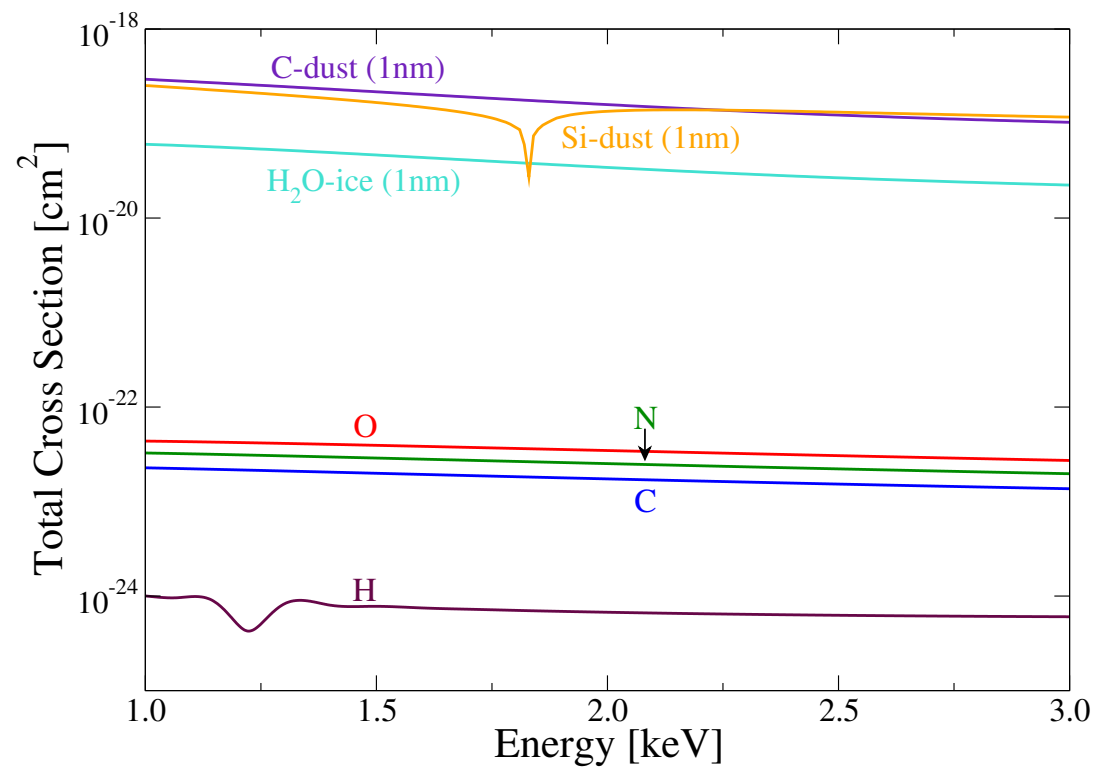

Fig. 3.12: Total cross sections for X-ray scattering from cometary gas and dust.

$2 \mathrm{keV}$, showing very similar features in the energy range between 1 and $2 \mathrm{keV}$ with the smooth spectral maximum in the region of pronounced spectral peak in the cometary spectra at $1.3 \mathrm{keV}$. The similarity in the spectra shown in Fig. 3.11 implies that a different mechanism, other than CX, may be responsible for these hard X-rays. Our aim then was to investigate the scattering and fluorescence of solar X-rays by gas and dust in the cometary atmosphere as the main mechanism for emission of hard X-rays [95].

In modeling the scattered X-ray spectrum from the cometary atmosphere, scattering cross sections, both total and differential, were required for scattering due to neutral gases as well as nanoparticles. The calculated Mie cross sections from Section 3.1 were utilized for nanoparticles consisting of silicon, carbon, and ice with grain sizes from 1 to $10 \mathrm{~nm}$ to determine the scattered solar X-ray spectrum from nanoparticles in the cometary atmosphere. Cross sections of elastic photon scattering and fluorescence 


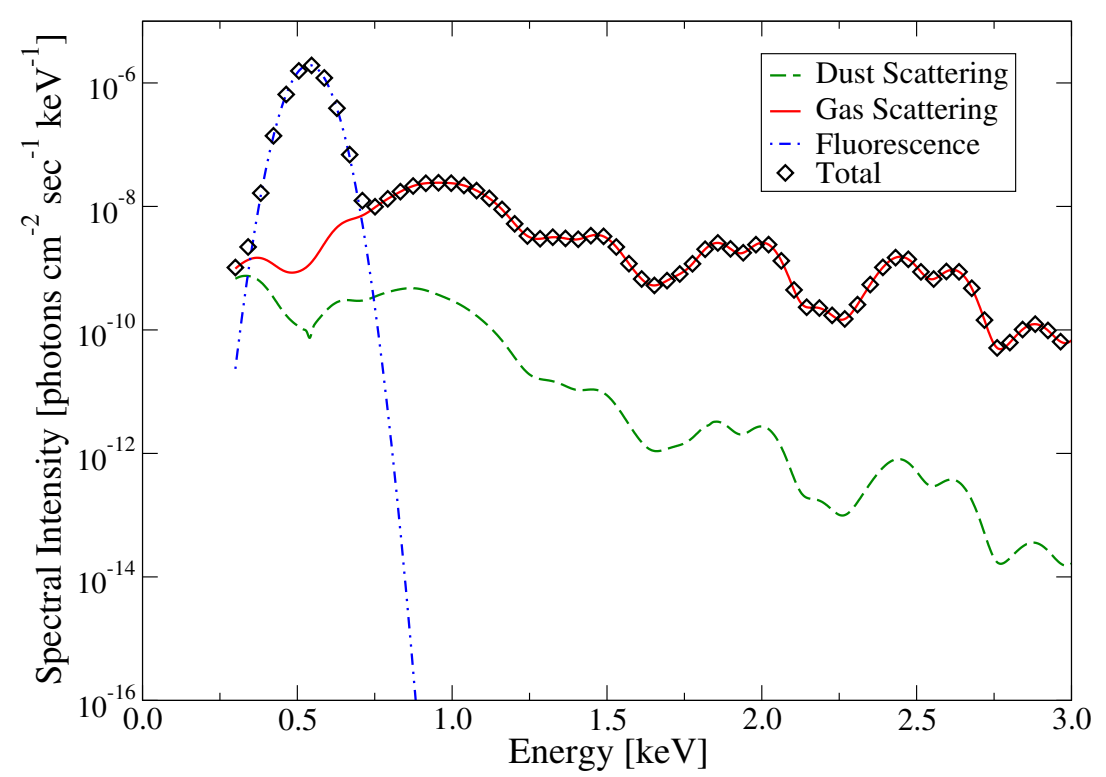

Fig. 3.13: An average X-ray spectrum emitted from a cometary atmosphere due to scattering and fluorescence.

for atomic hydrogen, oxygen, nitrogen, and carbon were obtained from reported laboratory experiments $[114,115]$. Fig. 3.12 displays the total cross sections for scattering of X-ray from the cometary gases and $1 \mathrm{~nm}$ nanoparticles for photon energies ranging from 1 and $3 \mathrm{keV}$ [95]. It is clear from Fig. 3.12 that the nanoparticles contribute to a much larger extent to X-ray scattering as compared to atomic scattering with the total cross sections being roughly 4 orders of magnitude larger for nanoparticles than for atomic species.

To determine the actual cometary X-ray spectrum, a model cometary atmosphere was used which assumed spherical symmetry in the gas and dust distributions $[111,116,40]$. The observation of comet Ikeya-Zhang by the Chandra space telescope was modeled using using the proper spatial properties and solar conditions at the time of 
observation [93,95]. Eq. 3.11 may be simplified for this system as the physical extent of the cometary atmosphere is several orders of magnitude smaller than the Earth-comet distance resulting in the scattered intensity ratio

$$
\frac{I_{s}(\lambda)}{I_{R_{0}}(\lambda)}=\frac{R_{0}^{2}}{r_{c}^{2}\left|r_{c}-R_{0}\right|^{2}} \sum_{j}\left|f_{j}\left(\lambda, \theta_{s c}\right)\right|^{2} N_{j}
$$

with $r_{c}$ being the detector-comet distance, $\left|f_{j}\left(\lambda, \theta_{s c}\right)\right|^{2}$ being the differential cross section for the $j^{\text {th }}$ atomic or nanoparticle species, and $N_{j}$ being the number of $j^{\text {th }}$ scatterers [95]. Using the spherically symmetric gas and dust distributions $[111,116,40]$, the scattered intensity due to nanoparticle scattering, gas scattering, and fluorescence was calculated with contributions from each process shown in Fig. 3.13. Utilizing the model gas and dust distributions, extrapolated from the observations of micron-size grains, the nanoparticle scattering component is between 2 and 4 orders of magnitude smaller than the gas scattering in the photon energy range from 1 to $3 \mathrm{keV}$ as seen in Fig. 3.13 despite the fact that the total scattering cross sections are much larger for nanoparticle scattering in this energy regime, Fig. 3.12. This is due to much lower nanoparticle densities in the model cometary atmosphere as compared to gas densities, leading to higher scattering contributions from the neutral gases. Nevertheless, there is no direct information about the density of ice and dust nanoparticles in the cometary atmosphere since these particles can not be seen in optical or infrared radiation. Real densities of ice and dust nanoparticles may be orders of magnitude higher than the extrapolated values which were extracted from observations of micron-size dust grains.

Direct comparison between observational data from Chandra and calculated results from Eq. 3.14 are shown in Fig. 3.14. Despite having similar spectra shape features, 


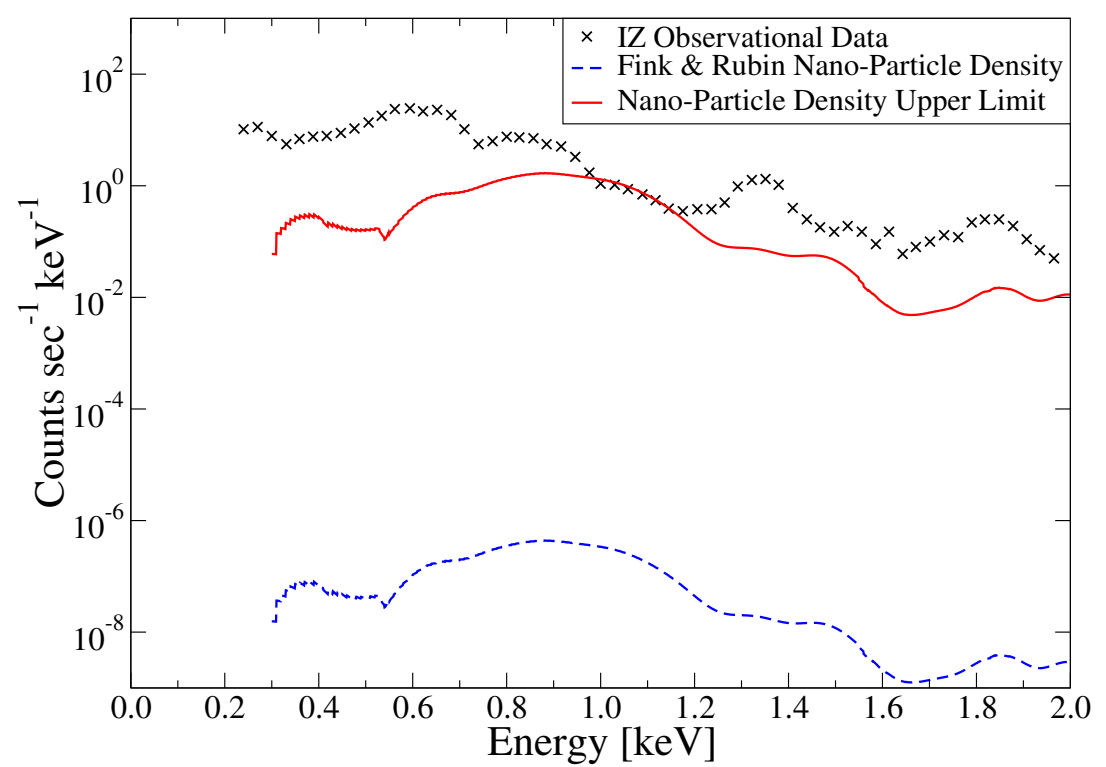

Fig. 3.14: A comparison of total spectral intensity contributions from nano-particle scattering to observational data of Ikeya-Zhang [93]. The calculated scattered X-ray spectrum is shown using a model dust distribution [116] as well as with an upper limit model assuming all gas production results in nanoparticles. 
the calculated X-ray spectrum shown in Fig. 3.14 is several orders of magnitude less intensive than that observed by Chandra [95]. The results shown in Fig. 3.14 with a blue dashed curve were calculated using nanoparticle densities which had been extrapolated from micron-size particles down to nano-size particles. It is entirely possible that nanoparticles, especially small ice particles, are more abundant than predicted by such a scaling technique. We therefore investigated the upper limit of nanoparticle densities by setting the density of nanoparticles to that of the neutral gas. This limit is realistic for dusty comets with the dust to gas production rates larger than 1. For example, recently observed dust comets with the dust to gas ratio larger than 4 [117] produces only emission of ice and dust particles with no detection of gas emission. The resulting scattered X-ray spectrum is shown in Fig. 3.14 with the solid red curve. Despite this upper limit on nanoparticle density, the calculated scattered X-ray intensity is still slightly smaller than was observed by Chandra. There is a bias in the Mie approximation towards smaller scattering angles at higher energies, resulting in the calculated spectrum decreasing faster than was observed $[102,95]$. This discrepancy can be attributed to the over simplification of modeling the nanoparticle grains as being homogeneous and spherical, demonstrating the importance of accurate geometrical and composition modeling of astrophysical nanoparticles. For example, angular distributions of high energy photons scattered by ultra small dust and ice particles with irregular shapes have a diffuse character and should be significantly more isotropic than spherical Mie scattering.

Results of X-ray scattering by a model cometary atmosphere reveal a similar spectral shape, yet large overall intensity from observational Chandra space telescope 
data [95]. The presence of nanoparticles within the cometary atmosphere may provide a significant scattering contribution if the production rate of nanoparticles is comparable to the gas production rate. Details of cometary nanoparticles are difficult to accurately observe, and analysis of scattering spectra may give deeper insight into the geometrical shape, composition, and spatial distributions of cometary nanoparticles. The scattering model utilized may be improved through use of accurate quantum mechanical cross sections for the nanoparticles instead of approximating all particles with the Mie model. 


\section{Conclusions}

The scattering of particles and radiation have been investigated in several different astrophysical environments including the Martian atmosphere, the interstellar gas, the heliosphere, and the cometary atmosphere.

Scattering parameters of fast atoms colliding with thermal atomic and molecular gases have been determined using ab initio quantum mechanical methods over a wide range of astrophysically important energies. Calculated differential cross sections were compared with experimental data with excellent agreement. In addition to quantum mechanical scattering parameters, an empirical scattering model has been developed for complex atom-molecule collisions which produces realistic scattering parameters when quantum parameters are not available.

The calculated scattering parameters were utilized in 3D, Monte Carlo transport simulations in the atmosphere of Mars and the interstellar atmosphere. Simulation results were compared with classical, hard sphere scattering models to illustrate the importance of accurate scattering parameters during transport and thermalization. Induced escape fluxes from the Mars atmosphere were calculated and compared to available theoretical predictions. Escaping atomic and molecular energy spectra were modeled for future in situ measurements. Additionally, fluxes of hot helium, originating from 
extra-solar sources, were obtained in the local interstellar medium. Steady state fluxes were compared with available in situ data to determine the significance of extra-solar hot atoms in our solar system, resulting in roughly $10 \%$ of background hot atoms originating from extra-solar sources. This $10 \%$ value may be considered as evidence of the cosmic background of He ENAs occupying our entire galaxy.

The scattering of X-rays by nanoparticles was also investigated. Scattering cross sections were calculated utilizing classical Mie models for nanoparticles consisting of carbon, silicon, and ice. Calculated scattering parameters were used to determine the scattered X-ray spectrum due to nanoparticles in the heliosphere as well as in a model cometary atmosphere. The scattered X-ray intensity due to heliospheric nanoparticles is lower than the X-ray background for normal solar conditions but may be visible during solar X-ray flares. Scattered X-ray spectra from a model cometary atmosphere was compared with in situ data resulting in very similar spectral shape for hard X-rays, between $1-3 \mathrm{keV}$.

The importance of accurate, energy-angular dependent atomic, molecular, and optical scattering parameters was demonstrated in several astrophysical environments. Accurate scattering parameters are necessary for realistic transport, thermalization, and energy-momentum transfer. New databases of accurate scattering parameters were developed for use in future modeling and predictions of next generation in situ observational instruments. 


\section{Bibliography}

[1] David Arnett. Supernovae and Nucleosynthesis. Princeton University Press, 1996.

[2] J. Fox and A Hać. Isotope Fractionation in the Photochemical Escape of $\mathrm{O}$ from Mars. Icarus, 208:176-191, 2010.

[3] B. Welsh and R. Shelton. The Trouble with the Local Bubble. Astrophysics and Space Science, 323:1-16, 2009.

[4] DJ McComas, HO Funsten, SA Fuselier, WS Lewis, E Möbius, and NA Schwadron. IBEX Observations of Heliospheric Energetic Neutral Atoms: Current Understanding and Future Directions. Geophysical Research Letters, 38(L18101), Sep 2011.

[5] R. Johnson, M. Combi, J. Fox, W. Ip, F. Leblanc, M. McGrath, V. Shematovich, D. Srobel, and J. Waite Jr. Exospheres and Atmospheric Escape. Space Science Reviews, 139:355-397, 2008.

[6] BD Shizgal and GG Arkos. Nonthermal Escape of the Atmosphere of Venus, Earth, and Mars. Reviews of Geophysics, 34(4):483-505, 1996.

[7] P. Brandt, D. Mitchell, E. Roelof, S. Krimigis, C. Paranicas, B. Mauk, J. Saur, and R. DeMajistre. ENA Imaging: Seeing the Invisible. Johns Hopkins APL Technical Digest, 26(2), 2005.

[8] E. Kallio, J. Luhmann, and S. Barabash. Charge Exchange Near Mars: The Solar Wind Absorption and Energetic Neutral Atom Production. Journal of Geophysical Reserach, 102(A10):183-197, 1997.

[9] G. Reeves, S. Morley, and G. Cunningham. Long-term variations in solar wind velocity and radiation belt electrons. Journal of Geophysical Research: Space Physics, 118:1-9, 2013.

[10] N. Lewkow, V. Kharchenko, and P Zhang. Energy Relaxation of Helium Atoms in Astrophysical Gases. The Astrophysical Journal, 756:57-68, 2012.

[11] N. Lewkow and V. Kharchenko. Precipitation of Energetic Neutral Atoms and Induced Non-Thermal Escape Fluxes from the Martian Atmosphere. The Astrophysical Journal, 790(2), 2014. 
[12] L. Landau and E. Lifshitz. Quantum Mechanics: Non-Relativistic Theory. Pergamon Press, 1991.

[13] P. Krstić and D. Schultz. Consistent Definitions for, and Relationships Among, Cross Sections for Elastic Scattering of Hydrogen Ions, Atoms, and Molecules. Physical Review A, 60(3), 1999.

[14] V. Fack and G. Berghe. (Extended) Numberov Method for Computing Eigenvalues of Specific Schrödinger Equations. Journal of Physics A, 20:4153-4160, 1987.

[15] W. Gropp, E. Lusk, and A. Skjellum. Using MPI: Portable Parallel Programming with the Message-passing Interface, Volume 1. MIT Press, 1999.

[16] E. Bich, R. Hellman, and E. Vogel. Ab initio Potential Energy Curve for the Helium Atom Pair and Thermophysical Properties of the Dilute Helium Gas. II. Thermophysical Standard Values for Low-density Helium. Molecular Physics, 105:3035-3049, 2007.

[17] H. Werner and P. Knowles. An Efficient Internally Contracted MulticonfigurationReference Configuration Interaction Method. The Journal of Chemical Physics, 89(9), Nov 1988.

[18] P. Knowles, C. Hampel, and H. Werner. Coupled Cluster Theory for High Spin, Open Shell Reference Wave Functions. The Journal of Chemical Physics, 99(7), Oct 1993 .

[19] A. Varandas. Generalized Uniform Singlet- and Triplet-Pair Extrapolation of the Correlation Energy to the One Electron Basis Set Limit. The Journal of Physical Chemistry A, 112(8):1841-1850, Feb 2008.

[20] Peng Zhang. Personal communication.

[21] S. Bovino, P. Zhang, F. Gianturco, A. Dalgarno, and V. Kharchenko. Energy Transfer in O Collisions with He Isotopes and Helium Escape from Mars. Geophysical Research Letters, 38(L02203), 2011.

[22] L. Wolniewicz. Nonadiabatic Energies of the Ground State of the Hydrogen Molecule. Journal of Chemical Physics, 103(5):1792-1799, 1995.

[23] D. O'Connor and J. Biersack. Comparison of Theoretical and Empirical Interatomic Potentials. Nuclear Instruments and Methods in Physics Research, B15:14$19,1986$.

[24] J. Biersack and J. Ziegler. Springer Series in Electrophysics, volume 10. Springer, Berlin, 1982.

[25] D. Nitz, R. Gao, L. Johnson, K. Smith, and R. Stebbings. Absolute Differential Cross Sections for Very-small-angle Elastic Scattering in $\mathrm{He}+\mathrm{He}$ Collisions at keV Energies. Physical Review A, 35:4541-4547, 1987. 
[26] G. Smith, R. Gao, B. Lindsay, K. Smith, and R. Stebbings. Absolute Differential Cross Sections for the Scattering of Kilo-electron-volt O Atoms. Physical Review A, 53:1581-1588, 1996.

[27] D. Schafer, J. Newman, K. Smith, and R Stebbings. Differential Cross Sections for Scattering of 0.5-, 1.5-, and 5.0-keV Oxygen Atoms by He, N2, and O2. Journal of Geophysical Research, 92(A6):6107-6113, 1987.

[28] R. Gao, L. Johnson, K. Smith, and R. Stebbings. Collisions of keV-energy H Atoms with the Rare Gases: Absolute Differential Cross Sections at Small Angles. Physical Review A, 40:4914-4919, 1989.

[29] J. Newman, Y. Chen, K. Smith, and R. Stebbings. Differential Cross Sections for Scattering of 0.5-, 1.5-, and 5.0-keV Hydrogen Atoms by $\mathrm{He}, \mathrm{H}_{2}, \mathrm{~N}_{2}$, and $\mathrm{O}_{2}$. Journal of Geophysical Research, 91(A8):8947-8954, 1986.

[30] R. Cabrera-Trujillo, J. Sabin, Y. Öhrn, and E. Deumens. Direct Differential-crosssection Calculations for Ion-atom and Atom-atom Collisions in the keV Range. Physical Review A, 61(032719), 2000.

[31] P. Krstić and D. Schultz. Elastic Scattering and Charge Transfer in Slow Collisions: Isotopes of $\mathrm{H}$ and $\mathrm{H}^{+}$Colliding with Isotopes of $\mathrm{H}$ and with He. Journal of Physics B: Atomic Molecular and Optical Physics, 32:3485-3509, 1999.

[32] I Amdur and E Mason. Scattring of High Velocity Neutral Particles, VIII, H-He. Journal of Chemical Physics, 25:630-632, 1956.

[33] Y Belyaev and V Leonas. Interaction Potentials of $\mathrm{H}$ and $\mathrm{He}$ atoms and $\mathrm{H}_{2}$ Molecules. Soviet Physics, Doklady, 12:233-235, 1967.

[34] F. Smith, R. Marchi, and K. Dedrick. Impact Expansions in Classical and Semiclassical Scattering. Physical Review, 150(1):79-92, 1966.

[35] J. Newman, K. Smith, and R. Stebbings. Differential Scattering Cross Sections for Collisions of 0.5-, 1.5-, and 5.0-keV Helium Atoms with $\mathrm{He}, \mathrm{H}_{2}, \mathrm{~N}_{2}$, and $\mathrm{O}_{2}$. Journal of Geophysical Reseearch, 90(A11):45-54, 1985.

[36] J. Fox and A Hać. Photochemical Escape of Oxygen from Mars: A Comparison of the Exobase Approximation to a Monte Carlo Method. Icarus, 204:527-544, 2009 .

[37] U. Feldman, U. Schühle, K. Widing, and J. Laming. Coronal Compostion above the Solar Equator and the North Pole as Determined from Spectra Acquired by the Sumer Instrument on SOHO. The Astrophysical Journal, 505:999-1006, 1998.

[38] V. Krasnopolsky. Mars' Upper Atmosphere and ionosphere at Low, Medium, and high Solar Activities: Implications for Evolution of Water. Journal of Geophysical Research, 107(E12):5118-5129, 2002. 
[39] J. Wallace and P. Hobbs. Atmospheric Science, Second Edition: An Introductory Survey. Academic Press, 2006.

[40] M. Rubin, V. Tenishev, M. Combi, K. Hansen, T. Gombosi, K. Altwegg, and H. Balsiger. Monte Carlo Modeling of Neutral Gas and Dust in the Coma of Comet 1P/Halley. Icarus, 213:655-677, 2011.

[41] J. Cui, R. Yelle, D. Strobel, C. Müller-Wodarg, D. Snowden, T. Koskinen, and M. Galand. The $\mathrm{CH}_{4}$ Structure of Titan's Upper Atmosphere Revisited. Journal of Geophysical Research, 117(E11006), 2012.

[42] B. Bransden and M. McDowell. Charge Exchange and the Theory of Ion-Atom Collisions. Clarendon Press, 1992.

[43] M. Aellig and A. Lazarus. The Solar Wind Helium Abundance: Variation with Wind Speed and the Solar Cycle. Geophysical Research Letters, 28(14):2767-2770, 2001.

[44] A. Dalgarno and D. Layzer. Spectroscopy of Astrophysical Plasmas. Cambridge University Press, 1987.

[45] J. Slavin and P. Frisch. The Boundary Conditions of the Heliosphere: Photoionization Models Constrained by Interstellar and in situ Data. Astronomy EG Astrophysics, 491:53-68, 2008.

[46] P. Frisch, S. Redfield, and J. Slavin. The Interstellar Medium Surrounding the Sun. The Annual Review of Astronomy and Astrophysics, 49:237-279, 2011.

[47] B. Lindsay and R. Stebbings. Charge Transfer Cross Sections for Energetic Neutral Atom Data Analysis. Journal of Geophysical Research, 110(A12213), 2005.

[48] C. Barnett. Collisions of $\mathrm{H}, \mathrm{H}_{2}, \mathrm{He}$, and Li Atoms and Ions with Atoms and Molecules. In H. Hunter and M. Kirkpatrick, editors, Atomic Data for Fusion, volume 1. Oak Ridge National Laboratory: Controlled Fusion Atomic Data Center, 1990 .

[49] T. Kusakabe, R. Buenker, and M. Kimura. Charge Transfer Processes in Collisions of $\mathrm{H}^{+}$Ions with $\mathrm{H}_{2}, \mathrm{D}_{2}, \mathrm{CO}, \mathrm{CO}_{2}, \mathrm{CH}_{4}, \mathrm{C}_{2} \mathrm{H}_{2}, \mathrm{C}_{2} \mathrm{H}_{6}$, and $\mathrm{C}_{3} \mathrm{H}_{8}$ Molecules below $10 \mathrm{keV}$. In R. Clark, editor, Atomic and Plasma-Material Interaction Data for Fusion, volume 10. International Atomic Energy Agency, 2002.

[50] J. Greenwood, A. Chutjian, and S. Smith. Measurements of Absolute, Single Charge-Exchange Cross Sections of $\mathrm{H}^{+}, \mathrm{He}^{+}$and $\mathrm{He}^{2+}$ with $\mathrm{H}_{2} \mathrm{O}$ and $\mathrm{CO}_{2}$. The Astrophysical Journal, 529:605-609, 2000.

[51] R. Gao, L. Johnson, D. Schafer, J. Newman, K. Smith, and R. Stebbings. Absolute Differential Cross Sections for Small-angle $\mathrm{He}^{+}-\mathrm{He}$ Elastic and Charge-transfer Scattering at keV Energies. Physical Review A, 38(6):2789-2793, 1988. 
[52] L. Johnson, R. Gao, C. Hakes, K. Smith, and R. Stebbings. Direction and Chargetransfer Scattering of $\mathrm{keV}$-energy $\mathrm{H}^{+}$and $\mathrm{He}^{+}$Projectiles from Rare-gas Atoms to Obtain Small-angle absolute Differential Cross Sections. Physical Review A, 40(9):4920-4925, 1989.

[53] R. Gao, L. Johnson, C. Hakes, K. Smith, and R. Stebbings. Collisions of Kiloelectron-volt $\mathrm{H}^{+}$and $\mathrm{He}^{+}$with Molecules at Small Angles: Absolute Differential Cross Sections for Charge Transfer. Physical Review A, 41(11):5929-5933, 1990.

[54] J. Ladd. Large-Scale Computational Analysis of National Animal Identification System Mock Data, Including Traceback and Trace Forward. PhD thesis, Colorado State University, Fort Collins, CO, 2008.

[55] D. Peplow. Direction Cosines and Polarization Vectors for Monte Carlo Photon Scattering. Nuclear Science and Engineering, 131:132-139, 1999.

[56] Herbert Goldstein, Charles P. Poole, and John L. Safko. Classical Mechanics (3rd Edition). Addison Wesley, 2001.

[57] S. Butler and M. Buckingham. Energy Loss of a Fast Ion in a Plasma. Physical Review, 126(1), 1962.

[58] Alexander Fridman. Plasma Chemistry. Cambridge University Press, 2012.

[59] P. Zhang, V. Kharchenko, A. Dalgarno, Y. Matsumi, T. Nakayama, and K. Takahashi. Approach to Thermal Equilibrium in Atomic Collisions. Physical Review Letters, 100(103001), 2008.

[60] V. Kharchenko, J. Tharamel, and A. Dalgarno. Kinetics of Thermalization of Fast Nitrogen Atoms beyond the Hard Sphere Approximation. Journal of Atmospheric and Solar-Terrestrial Physics, 59(1):107-115, 1997.

[61] H. Gröller, V. Shematovich, H. Lichtenegger, H. Lammer, M. Pfleger, Y. Kulikov, W. Macher, U. Amerstorfer, and H. Biernat. Venus' Atomic Hot Oxygen Environment. Journal of Geophysical Research, 115(E12017), 2010.

[62] D. Hunten. Thermal and Nonthermal Escape Mechanisms for Terrestrial Bodies. Planetary Space Science, 30(8):773-783, 1982.

[63] T. Owen, K. Biemann, D. Rushneck, J. Biller, D. Howarth, and A. Lafleur. The Composition of the Atmosphere at the Surface of Mars. Journal of Geophysical Research, 82(28):4635-4639, 1977.

[64] V. Shematovich, G. Tsvetkov, M. Krestyanikova, and M. Marov. Stochastic Models of Hot Planetary and Satellite Coronas: Total Water Loss in the Martian Atmosphere. Solar System Research, 41(2):103-108, 2007.

[65] H. Lammer, E. Chassefiére, O Karatekin, A. Morschhauser, P. Niles, O. Mousis, P. Odert, U. M ostl, D. Breuer, V. Dehant, M. Grott, H. Gr oller, E. Hauber, and 
L. Pham. Outgassing History and Escape of the Martian Atmosphere and Water Inventory. Space Science Review, 174:113-154, 2013.

[66] J. Fox and A. Hać. The Escape of O from Mars: Sensitivity to the Elastic Cross Sections. Icarus, 228:375-385, 2014.

[67] E. Kallio and S. Barabash. Atmospheric Effects of Precipitating Energetic Hydrogen Atoms on the Martian Atmosphere. Journal of Geophysical Research, 106(A1):165-177, 2001.

[68] V. Shematovich. Stochastic Models of Hot Planetary and Satellite Coronas. Solar System Research, 38(1):28-38, 2004.

[69] M. Krest'yanikova and V. Shematovich. Stochastic Models of Hot Planetary and Satellite Coronas: A Photochemical Source of Hot Oxygen in the Upper Atmosphere of Mars. Solar System Research, 39(1):22-32, 2005.

[70] Agustin Sanchez-Lavega. An Introduction to Planetary Atmospheres. Taylor \& Francis, 2010.

[71] J. Phillips, S. Bame, A. Barnes, B. Barraclough, W. Feldman, B. Goldstein, J. Gosling, G. Hoogeveen, D. McComas, M. Neugebauer, and S. Suess. Ulysses Solar Wind Plasma Observations from Pole to Pole. Geophysical Research Letters, 22(23):3301-3304, 1995.

[72] A. Bondi. Van der Waals Volumes and Radii. Physical Chemistry, 68(3):441-451, 1964.

[73] N. Balakrishnan, V. Kharchenko, and A. Dalgarno. Translational Energy Relaxation of Hot $\mathrm{O}\left({ }^{1} \mathrm{D}\right)$ Atoms. Journal of Chemical Physics, 103:3999-4001, 1999.

[74] P. Zhang, V. Kharchenko, M. Jamieson, and A. Dalgarno. Energy Relaxation in Collisions of Hydrogen and Deuterium with Oxygen Atoms. Journal of Geophysical Research, 114(A07101), 2009.

[75] D. McComas, F. Allegrini, P. Bochsler, M. Bzowski, E. Christian, G. Crew, R. DeMajistre, H. Fahr, H. Fichtner, P. Frisch, H. Funsten, S. Fuselier, G. Gloeckler, M. Gruntman, J. Heerikhuisen, V. Izmodenov, P. Janzen, P. Knappenberger, S. Krimigis, H. Kucharek, M. Lee, G. Livadiotis, S. Livi, R. MacDowall, D. Mitchell, E. Möbius, T. Moore, N. Pogorelov, D. Reisenfeld, E. Roelof, L. Saul, N. Schwadron, P. Valek, R. Vanderspek, P. Wurz, and G. Zank. Global Observations of the Interstellar Interaction from the Interstellar Boundary Explorer (IBEX). Science, 326:959-962, 2009.

[76] D. McComas, M. Bzowski, P. Frisch, G. Crew, M. Dayeh, R. DeMajistre, H. Funsten, S. Fuselier, M. Gruntman, P. Janzen, M. Kubiak, G. Livadiotis, E. Möbius, D. Reisenfeld, and N. Schwadron. Evolving Outer Heliosphere: Large-scale Stability and Time Variations Observed by the Interstellar Boundary Explorer. Journal of Geophysical Research, 115(A09113), 2010. 
[77] D. McComas, M. Dayeh, F. Allegrini, M. Bzowski, R. DeMajistre, K. Fujiki, H. Funsten, S. Fuselier, M. Gruntman, P. Janzen, M. Kubiak, H. Kucharek, G. Livadiotis, E. Möbius, D. Reisenfeld, M. Reno, N. Schwadron, J. Sokol, and M. Tokumaru. The First Three Years of IBEX Observations and our Evolving Heliosphere. The Astrophysical Journal, 203(1), 2012.

[78] F. Shu. The Physical Universe: An Introduction to Astronomy. University Science Books, 1982.

[79] R. Lallement, B. Welsh, J. Vergely, F. Crifo, and D. Sfeir. 3D Mapping of the Dense Interstellar Gas around the Local Bubble. Astronomy and Astrophysics, 411:447-464.

[80] N. Lewkow and V. Kharchenko. Relaxation and Transport of ENAs in the Interstellar Medium. San Francisco, 2012. American Geophysical Union.

[81] N. Lewkow and V. Kharchenko. Relaxation and Transport of ENAs Produced Between the Interstellar Gas and Solar Wind. San Francisco, 2013. American Geophysical Union.

[82] P. Swaczyna, S. Grzedzielski, and M. Bzowski. Assessment of Energetic Neutral He Atom Intensities Expected from the IBEX Ribbon. astro-ph.SR, arXiv:1310.8438v2, 2014.

[83] P. Frisch. How Local is the Local Interstellar Magnetic Field? arXiv astro-ph, 1111.3675v1, 2011.

[84] RECON. The One Hundred Nearest Star Systems, 2012.

[85] W. Maciel. Hydrodynamics and Stellar Winds. Springer, 2014.

[86] F. Allegrini, G. Crew, D. Demkee, H. Funsten, D. McComas, B. Randol, B. Rodriguez, N. Schwadron, P. Valek, and S. Weidner. The IBEX Background Monitor. Space Science Reviews, 146, 2008.

[87] R. Giacconi. Nobel Lecture: The Dawn of X-ray Astronomy. Reviews of Modern Physics, 75:995-1010, 2003.

[88] B. Draine. Interstellar Dust Grains. Annual Review of Astonomy and Astrophysics, 41, 2003.

[89] B. Draine. Scattering by Interstellar Dust Grains. II. X-Rays. The Astrophysical Journal, 598:1026-1037, 2003.

[90] R. Smith. X-Ray Dust Scattering at Small Angles: The Complete Halo around GX13+1. The Astrophysical Journal, 681:343-349, 2008.

[91] R. Smith, T. Dame, E. Costantini, and P. Predehl. The X-Ray Halo of GX 5-1. The Astrophysical Journal, 648:452-460, 2006. 
[92] R. Smith, R. Edgar, and R. Shafer. The X-Ray Halo of GX 13+1. The Astrophysical Journal, 581:562-569, 2002.

[93] I. Ewing, D. Christian, D. Bodewits, K. Dennerl, C. Lisse, and S. Wolk. Emission Lines Between 1 and $2 \mathrm{keV}$ in Cometary X-ray Spectra. The Astrophysical Journal, $763(66), 2013$.

[94] N. Schwadron and T. Cravens. Implications of Solar Wind Composition for Cometary X-Rays. The Astrophysical Journal, 544:558-566, 2000.

[95] B. Snios, N. Lewkow, and V. Kharchenko. Cometary Emission Induced by Scattering and Fluorescence of Hard Solar X-rays. Astronomy 8 Astrophysics, 2014.

[96] I. Newton. Opticks: Or a Treatise of the Reflections, Refractions, Inflections 8 Colours of Light. Dover Publications, 1730.

[97] R. Feynman. QED: The Strange Theory of Light and Matter. Princeton University Press, 1988.

[98] I. Mann. Interstellar dust in the solar system. Annual Review of Astronomy and Astrophysics, 48:173-203, 2010.

[99] A. Czechowski and I. Mann. Formation and Acceleration of Nano Dust in the Inner Heliosphere. The Astrophysical Journal, 714:89-99, 2010.

[100] Krasnopolsky:1997. On the Nature of Soft X-Ray Radiation in Comets. Icarus, 128:368-385, 1997.

[101] D. Schlegel, D. Finkbeiner, and M. Davis. Maps of dust infrared emission for use in estimation of reddening and cosmic microwave background radiation foregrounds. The Astrophysical Journal, 500:525-553, 1998.

[102] H. van de Hulst. Light Scattering by Small Particles. Dover Publications, 1981.

[103] H. Du. Mie-scattering Calculation. Applied Optics, 43(9):1951-1956, 2004.

[104] B. Henke, E. Gullikson, and J. Davis. X-Ray Interactions: Photoabsorption, Scattering, Transmission, and Reflection at $\mathrm{E}=50-30,000 \mathrm{eV}, \mathrm{Z}=1-92$. Atomic Data and Nuclear Data Tables, 54:181-342, 1993.

[105] J. Hubbell, P. Trehan, N. Singh, B. Chand, D. Mehta, M. Garg, R. Garg, S. Singh, and S. Purl. A Review, Bibliography, and Tabulation of K, L, and Higher Atomic Shell X-Ray Fluorescence Yields. Journal of Chemical Reference Data, 23(2):339364, 1994.

[106] N. Meyer-Vernet, M. Maksimovic, A. Czechowski, I. Mann, I. Zouganelis, K. Goetz, M. Kaiser, O. Cyr, J. Bougeret, and S. Bale. Dust Detection by the Wave Instrument on STEREO: Nanoparticles Picked up by the Solar Wind? Solar Physics, 256:463-474, 2009. 
[107] V. Vasylyev, A. Kalinichenko, and S. Vasylyev. Attogram Dust Grains in the Solar System. Astronomy and Astrophysics, 415:781-790, 2004.

[108] V. Kharchenko and N. Lewkow. Charge-exchange and x-ray processes with nanodust particles. In I. Mann, editor, Nanodust in the Solar System: Discoveries and Interpretations, volume 385 of Astrophysics and Space Science Library, pages 179-194. Springer, 2012.

[109] A. Bhardwaj, R. Elsner, G. Gladstone, T. Cravens, C. Lisse, K. Dennerl, G. Branduardi-Raymont, B. Wargelin, J. Waite, I. Robertson, N. Ostgaard, P. Beiersdorfer, S. Snowdent, and V. Kharchenko. X-rays from Solar System Objects. Planetary and Space Science, 55:1135-1189, 2007.

[110] C. Lisse, K. Dennerl, J. Englhauser, M. Harden, F. Marshall, M. Mumma, R. Petre, J. Pye, M. Ricketts, J. Schmitt, J. Trumper, and R West. Discovery of X-ray and Extreme Ultraviolet Emission from Comet C/Hyakutake 1996 B2. Science, 274(5285):205-209, 1996.

[111] T Cravens. Comet Hyakutake X-ray Source: Charge Transfer of Solar Wind Heavy Ions. Geophysical Research Letters, 24(1):105-108, 1997.

[112] R. von Steiger, N. Schwadron, L. Fisk, J. Geiss, G. Gloeckler, S. Hefti, B. Wilken, R. Wimmer-Schweingruber, and T. Zurbuchen. Composition of Quasi-stationary Solar Wind Flows from Ulysses/Solar Wind Ion Composition Spectrometer. Journal of Geophysical Research, 105(A12):27217-27238, 2000.

[113] G. Branduardi-Raymont, A. Bhardwaj, R. Elsner, G. Gladstone, G. Ramsay, P. Rodriguez, R. Soria, Waite J., and T. Cravens. Latest results on the Jovian disk X-rays from XMM-Newton. Planetary and Space Science, 55:1126-1134, 2007.

[114] C. Chantler. Theoretical Form Factor, Attenuation and Scattering Tabulation for $\mathrm{Z}=1-92$ from $\mathrm{E}=1-10 \mathrm{eV}$ to $\mathrm{E}=0.4-1.0 \mathrm{MeV}$. Journal of Physical Chemistry Reference Data, 24(1), 1995.

[115] M. Berger, J. Hubbell, S. Seltzer, J. Chang, J. Coursey, R. Sukumar, D. Zucker, and K. Olsen. XCOM: Photon Cross Section Database (version 1.5), May 2010.

[116] U. Fink and M. Rubin. The Calculation of Af $\rho$ and Mass Loss Rate for Comets. Icarus, 221:721-734, 2012.

[117] B. Yang, J. Keane, K. Meech, T. Owen, and R. Wainscoat. Multi-Wavelength Observations of Comet C/2011 L4 (PAN-STARRS). The Astrophysical Journal Letters, 784(L23), 2014. 